\author{
Universidade de São Paulo \\ Instituto de Astronomia, Geofísica e Ciências Atmosféricas
}

Departamento de Astronomia

Christiano Santos Rennó

\title{
Abundâncias químicas a partir de espectros integrados de 47 Tucanae (NGC 104)
}

São Paulo 

Christiano Santos Rennó

\section{Abundâncias químicas a partir de espectros integrados de 47 Tucanae (NGC 104)}

Dissertação apresentada ao Departamento de Astronomia do Instituto de Astronomia, Geofísica e Ciências Atmosféricas da Universidade de São Paulo como requisito parcial para a obtenção do título de Mestre em Ciências. Versão corrigida. O original encontra-se na Unidade.

Área de Concentração: Astronomia Orientador(a): Prof. $\left(^{a}\right)$ Dr. $\left(^{a}\right)$ Beatriz L. S. Barbuy

São Paulo 

À Malu, Zeca e Dai, que seguraram minha mão por todos esses anos 



\section{Agradecimentos}

Aos meus pais, Malu e Zeca, que sempre acreditaram e me apoiaram em todos os passos que dei na minha história. Que sempre me receberam com um abraço, nos momentos mais felizes e nos momentos mais difíceis. Eu sempre amarei vocês. Obrigado por estarem comigo;

À minha irmã, Dai, que sempre se preocupou em me mostrar o melhor caminho, e que sempre acreditou em mim e que me fez acreditar também. Você sempre foi meu maior exemplo. Eu amo você ;

Ao Paulo, meu cunhado, com quem pude ter diversas discussões frutíferas e que me provocou muitas vezes a olhar as coisas por outras perspectivas. Sou muito grato pelos seus toques e conselhos. Muito obrigado;

À Doutora Beatriz Barbuy, que me deu a oportunidade de desenvolver esse trabalho e que foi sempre solícita e que tanto me ensinou em nossas discussões;

Às Doutoras Tatiana Moura e Marina Trevisan, que deram contribuições essenciais para o desenvolvimento desse trabalho, e que proporcionaram discussões importantes que contribuíram para o meu conhecimento em Astronomia;

Aos Doutores Jorge Meléndez e Xavier Barcons, aos meus colegas da turma de Astrofísica Observacional e a todos que contribuíram para a viagem ao ESO. Foi uma oportunidade incrível que não seria possível sem todo esse apoio, e agradeço à Bruno Dias, que nos guiou e que dividiu conosco sua experiência durante nossa estadia no ESO-Paranal;

Aos colegas Antonio, Heitor, Julian, Larissa e Lívia, pelo companheirismo e pelas risadas e inúmeras discussões na F308, vou sentir saudades;

A todos os meus amigos que estiveram comigo nessa longa caminhada. Sem vocês, tudo seria muito mas complicado; 
À CAPES, pelo apoio financeiro;

Ao IAG, seus funcionarios e docentes, e à USP;

À Amanda, que tanto me ensinou enquanto esteve ao meu lado nessa longa jornada, e que, mesmo ao partir, fez com que eu olhasse para meus defeitos e me tornasse um ser humano melhor. Muito obrigado, e muito boa sorte em seu caminho. 
"What an astonishing thing a book is. It's a flat object made from a tree with flexible parts on which are imprinted lots of funny dark squiggles. But one glance at it and you're inside the mind of another person, maybe somebody dead for thousands of years. Across the millennia, an author is speaking clearly and silently inside your head, directly to you.

Writing is perhaps the greatest of human inventions, binding together people who never knew each other, citizens of distant epochs. Books break the shackles of time. A book is proof that humans are capable of working magic"

Carl Sagan em Cosmos, Episódio 11 (A Persistência da Memória)

"Unaware of where my heart would flow, I was wading in the undertow."

Nick Kingswell em "Undertow" 



\section{Resumo}

Estudos de espectros integrados de aglomerados globulares bem conhecidos da Via Láctea podem ser usados como modelos para análises de objetos mais distantes e menos luminosos. O aglomerado globular 47 Tucanae está entre um dos mais estudados e observados, devido a sua proximidade. O objetivo principal deste trabalho é estudar em detalhes o espectro integrado do 47 Tuc, para se obter uma lista de linhas confiável para estudos futuros desse objeto e de outros aglomerados globulares.

Neste trabalho, o espectro de 47 Tucanae é reproduzido através da construção de um espectro sintético, que leva em consideração abundâncias elementais individuais, e os resultados obtidos são comparados com estudos prévios.

Foi reproduzido o espectro integrado no intervalo 4500 - $9000 \AA$, e foram calculadas as abundâncias dos elementos $\mathrm{Na}, \mathrm{Mg}, \mathrm{Al}, \mathrm{Si}, \mathrm{Ca}, \mathrm{Ti}, \mathrm{Ba}$, e Eu, por meio de ajustes de

linhas individuais. É apresentada uma lista de linhas que são adequadas para a derivação de abundâncias. Adotando as abundâncias calculadas, foi possível o ajuste das asas dos bem conhecidos tripletos de MgI e CaII. Por fim, foi possível testar a presença de múltiplas populações estelares no 47 Tuc a partir da presença de enriquecimento de Na.

Os resultados obtidos para as abundâncias são compatíveis com as abundâncias previamente apresentadas em trabalhos anteriormente publicados, que se utilizam de diferentes técnicas para a análise dos espectros obtidos. Uma discordância mais significativa foi observada entre as abundâncias de $\mathrm{Na}$, e pode ter sido causada por efeitos devidos a múltiplas populações estelares presentes no aglomerado.

O método utilizado para a construção de espectros integrados sintéticos nesse trabalho pode ser utilizado para cálculos de abundâncias de espectros integrados empíricos. Esse resultado pode ser útil para análises de aglomerados mais distantes e menos luminosos, e 
posteriormente para análises de galáxias externas. 


\section{Abstract}

Studies of integrated spectra of well-known Milky Way globular clusters can be tested as templates to allow reliable studies of faint and distant objects. 47 Tucanae is among the most studied and observed globular clusters, given its proximity. The aim of this work to study in detail the integrated spectrum of 47 Tucanae, in order to have a list of reliable lines for future studies.

In this work, the spectrum of 47 Tucanae is reproduced by computing synthetic spectra, taking into account individual element abundances. The results are compared with other methods. We reproduce the integrated spectrum in the full range 4500-9000 $\AA$ and derive abundances from individual lines of $\mathrm{Na}, \mathrm{Mg}, \mathrm{Al}, \mathrm{Si}, \mathrm{Ca}, \mathrm{Ti}, \mathrm{Ba}$, and $\mathrm{Eu}$. We report a list of lines that are suitable for abundance derivation. Adopting these abundances we are able to fit the wings of the well-known triplets of MgI and CaII. Finally, the effect of multiple stellar populations through enhanced $\mathrm{Na}$ abundances are tested.

Element abundances obtained are compatible with previous literature abundances, from different methods. A larger spread of $\mathrm{Na}$ abundances could be due to the effect of multiple stellar populations. The method here applied of building integrated synthetic spectra, can be used to derive abundances from observed integrated spectra. This can be useful for the analysis of distant and faint clusters. These results can be applied in future analyses of integrated spectra from external galaxies. 



\section{Lista de Figuras}

1.1 Imagem do 47 Tuc . . . . . . . . . . . . . . . . . . . . . 23

2.1 Exemplo de isócrona criada pela função stpars. . . . . . . . . . . . . . . . . 31

3.1 Espectros observado e sintético para as linhas de MgI em 5528.405 e $8806.756 \AA$ [36

3.2 Espectros observado e sintético para as linhas de AlI em 6696.185, 6696.788 e $6698.673 \AA \ldots \ldots \ldots \ldots \ldots \ldots$

3.3 Espectros observado e sintético para as linhas de SiI em 5948.55, 7405.79, 7415.96 e $7423.51 \AA \ldots \ldots \ldots \ldots \ldots$

3.4 Espectros observado e sintético para as linhas de CaI em 6102.723, 6122.217 ,6161.295, 6162.167, 6166.44 e 6169.044 e $6169.56 \AA \ldots$. . . . . . . . 39

3.5 Espectros observado e sintético para as linhas de CaI em 6439.08, 6455.605,

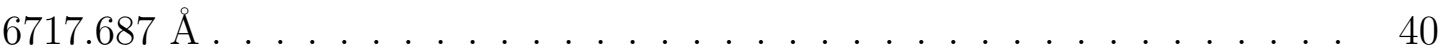

3.6 Espectros observado e sintético para as linhas de TiI em 5866.449, 5941.75, 5965.825 e $5978.539 \AA \ldots \ldots \ldots \ldots$

3.7 Espectros observado e sintético para as linhas de TiI em 6126.214, 6261.106, 6266.01 e $6556.077 \AA \ldots \ldots \ldots \ldots$

3.8 Espectros observado e sintético para a linha de TiI em $6743.127 \AA$ A . . . . 44

3.9 Espectros observado e sintético para as linhas de TiI em 5154.068, 5336.771, 5381.021 e $5418.751 \AA \ldots \ldots \ldots \ldots$

3.10 Espectros observado e sintético para as linhas de BaII em 5853.675, 6141.713 e $6496.9 \AA \ldots \ldots \ldots \ldots \ldots \ldots$

3.11 Espectros observado e sintético para a linha de EuII em 6645.064 A. . . . . 47

3.12 Linhas do tripleto do MgI localizadas em 5167.32, 5172.68 e $5183.604 \AA$. . 48 
3.13 Linhas do tripleto do CaII localizadas em 8498.18, 8542.089 e $8662.14 \AA$. .

3.14 Ajuste entre espectro observado e espectros sintéticos para diferentes valores de abundância de $\mathrm{Na}$, para as linhas de NaI localizadas em 5682.633,

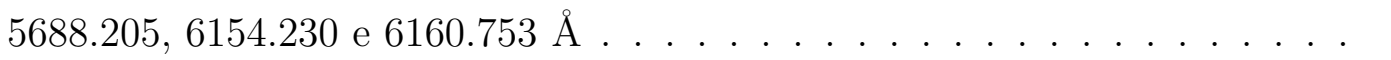

3.15 Ajuste entre espectro observado e espectros sintéticos para diferentes valores de abundância de $\mathrm{Na}$, para as linhas de $\mathrm{NaI}$ localizadas em 8183.256 e

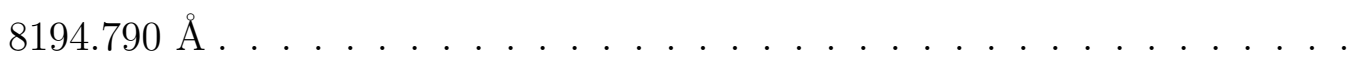

3.16 Comportamento de isócronas de acordo com E(B - V), módulo de distância, idade, metalicidade e abundância de He . . . . . . . . . . . . . 5 52

3.17 Espectros integrados para o 47 Tuc com abundâncias elementais idênticas e idades de 11 e 13 Gyrs, além de seus respectivos resíduos, no intervalo 5000 $-5500 \AA \ldots \ldots \ldots \ldots \ldots \ldots$. . . . . . . . . . . . . . . . . . .

3.18 Abundâncias versus Z para esse trabalho e resultados da literatura . . . . . 57

A.1 Comparação entre espectros sintético e observado, intervalo 4500 - $5500 \AA$

A.2 Comparação entre espectros sintético e observado, intervalo 5500 - $6500 \AA$

A.3 Comparação entre espectros sintético e observado, intervalo $6500-7500 \AA \quad 76$

A.4 Comparação entre espectros sintético e observado, intervalo 7500 - $8500 \AA \quad 77$

A.5 Comparação entre espectros sintético e observado, intervalo 8500 - $9000 \AA \quad 78$

B.1 Espectros sintéticos com idades de 11 e 13 Gyr e espectro observado sobrepostos, no intervalo $4500-5000 \AA . \quad \ldots \ldots$. . . . . . . . . 80

B.2 Espectros sintéticos com idades de 11 e 13 Gyr e espectro observado sobrepostos, no intervalo $5000-5500 \AA . \ldots \ldots 1$

B.3 Espectros sintéticos com idades de 11 e 13 Gyr e espectro observado sobrepostos, no intervalo $5500-6000 \AA$. . . . . . . . . . . . 82

B.4 Espectros sintéticos com idades de 11 e 13 Gyr e espectro observado sobrepostos, no intervalo $6000-6500 \AA . \ldots \ldots$

B.5 Espectros sintéticos com idades de 11 e 13 Gyr e espectro observado sobrepostos, no intervalo $6500-7000 \AA . \ldots \ldots$. . . . . . . . . 84

B.6 Espectros sintéticos com idades de 11 e 13 Gyr e espectro observado sobrepostos, no intervalo $7000-7500 \AA$. . . . . . . . . . . . . 
B.7 Espectros sintéticos com idades de 11 e 13 Gyr e espectro observado sobrepostos, no intervalo $7500-8000 \AA . \ldots \ldots 6$

B.8 Espectros sintéticos com idades de 11 e 13 Gyr e espectro observado sobrepostos, no intervalo $8000-8500 \AA . \ldots \ldots$. . . . . . . . . . 87

B.9 Espectros sintéticos com idades de 11 e 13 Gyr e espectro observado sobrepostos, no intervalo $8500-9000 \AA$. . . . . . . . . . . . 88 



\section{Lista de Tabelas}

1.1 Informações básicas do 47 Tucanae . . . . . . . . . . . . . . . 23

1.2 Metalicidades e abundâncias da literatura para o 47 Tuc . . . . . . . 27

2.1 Constantes atômicas para os Tripletos de MgI e CaII . . . . . . . . . . . 33

2.2 Idades da literatura (Gyr) para o 47 Tuc . . . . . . . . . . . . . . . . 34

3.1 Abundâncias médias calculadas por ajuste de linhas individuais . . . . . 53

3.2 Comparação de linhas com o trabalho de Colucci et al. (2017) . . . . . . 5 56

3.3 Lista de linhas analisadas . . . . . . . . . . . . . . . . 58 



\section{Sumário}

1. Introdução . . . . . . . . . . . . . . . . . . . . . . . . 21

1.1 Aglomerados Globulares . . . . . . . . . . . . . . . . 21

1.1.1 Estudos de Abundâncias do 47 Tuc . . . . . . . . . . . . . 23

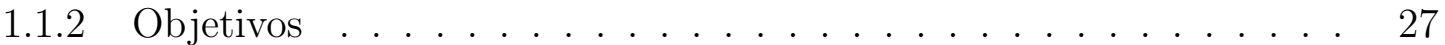

2. Metodologia . . . . . . . . . . . . . . . . . . . . . 29

2.1 Dados Observacionais . . . . . . . . . . . . . . . . . . . . . . 29

2.2 Espectros Sintéticos . . . . . . . . . . . . . . . . . . . . . . . . . . . 29

2.3 Valores Utilizados para o 47 Tuc . . . . . . . . . . . . . . . . 33

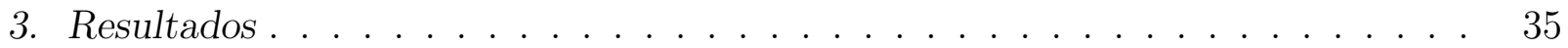

3.1 Ajustes de Linhas Individuais . . . . . . . . . . . . . 35

3.1 .1 Linhas de $\mathrm{Mg} \ldots \ldots \ldots \ldots \ldots$

3.1 .2 Linhas de $\mathrm{Al} \ldots \ldots \ldots \ldots$

3.1 .3 Linhas do $\mathrm{Si} \ldots \ldots \ldots \ldots$

3.1 .4 Linhas do $\mathrm{Ca} \ldots \ldots \ldots \ldots$

3.1 .5 Linhas do $\mathrm{Ti} \ldots \ldots \ldots \ldots$

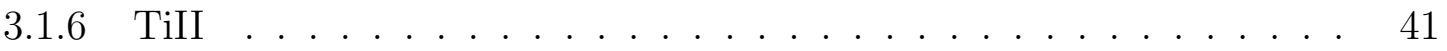

3.1 .7 Linhas do $\mathrm{Ba} \ldots \ldots \ldots \ldots$

3.1 .8 Linha do $\mathrm{Eu} \ldots \ldots \ldots \ldots \ldots$

3.2 Linhas fortes . . . . . . . . . . . . . . . . . 41

3.3 Múltiplas Populações Estelares em Aglomerados Globulares . . . . . . . . . 47

3.4 Abundâncias de $\mathrm{He} \ldots \ldots$. . . . . . . . . . . . . . . . . . 50

3.5 Efeitos da Idade . . . . . . . . . . . . . . . . . . . . . 50 
3.6 Discussão . . . . . . . . . . . . . . . . . . . . . . . . 53

3.6 .1 Incertezas associadas . . . . . . . . . . . 55

4. Conclusões . . . . . . . . . . . . . . . . . . . . . . 59

Referências .............................. 61

$\begin{array}{ll}\text { Apêndice } & 71\end{array}$

A. Comparação entre espectros do SynSSP e espectro observado em Usher et al. (2017) 73

B. Comparação entre espectros sintéticos com idades de 11 e 13 Gyr, e espectro empírico ............................... 79 
Capítulo 1

\section{Introdução}

\subsection{Aglomerados Globulares}

Um aglomerado globular é uma estrutura esférica, formada de múltiplas estrelas, fortemente conectadas pela gravidade, que orbitam um centro galáctico, e cuja densidade de estrelas aumenta quanto mais próximo do centro da estrutura. Na Via Láctea, aglomerados globulares são encontrados tanto no halo quanto na região do bojo galáctico, são consideravelmente mais velhos e mais densos em estrelas do que aglomerados abertos, os aglomerados globulares são os objetos mais velhos do universo. Existem por volta de 200 aglomerados globulares conhecidos na Via Láctea (Bica et al., 2019), e outras galáxias possivelmente possuem um número ainda maior: Andrômeda, por exemplo, pode ter cerca de 500 (Barmby e Huchra, 2001). Os mecanismos de formação de aglomerados globulares ainda não são bem entendidos, e mais recentemente, vastas evidências de que um aglomerado globular não é composto de apenas uma população estelar foram encontradas,vide os trabalhos de Milone et al. (2017), ou o review de Bastian e Lardo (2018). Esses resultados tornam ainda mais complexos os cenários de formação desse tipo de objeto.

A magnitude absoluta típica de um aglomerado globular (posteriormente citados como GCs, Globular Clusters, do inglês) é de cerca de $\mathrm{M}_{v} \sim-7.5$ (Harris, 1991), cerca de cinco magnitudes mais brilhante do que uma estrela gigante vermelha (RGB). Devido a essa característica, esses objetos podem ser observados a distâncias até dez vezes maiores do que RGBs. Mesmo com a nova geração de telescópios de 30-40 metros ainda não será possível observar estrelas individuais a distâncias de $10 \mathrm{Mpc}$ ou superiores, e espectros integrados são a opção mais viável para observações espectroscópicas de objetos distantes.

Espectros integrados de aglomerados globulares vêm se tornando interessantes temas 
de pesquisa desde os trabalhos de (Burstein et al., 1984) e (Bica, Alloin e Schmitt, 1994), onde foi apontado que o estudo desses objetos com esse tipo de técnica é importante para o melhor entendimento de populações estelares em galáxias, que são objetos mais complexos, compostos de várias populações estelares, enquanto aglomerados globulares são objetos mais simples, que por muito tempo foram entendidos como compostos de uma única população estelar. Embora exista ampla evidência de que GCs não sejam compostos de apenas uma população estelar (Milone et al., 2017), o estudo de GCs continua sendo crucial para o melhor entendimento de populações estelares.

O aglomerado globular 47 Tucanae (ou NGC 104, que posteriormente será citado como 47 Tuc) é um dos aglomerados mais próximos do Sol, juntamente com o M4 (NGC 6121), a uma distância $\mathrm{d}_{\circ} \sim 4.5 \mathrm{kpc}$ do centro da galáxia, além de ser o segundo mais brilhante no céu, com uma magnitude aparente $\mathrm{m}_{\mathrm{v}}=3.95$, é superado apenas por $\omega C$ en (Harris 1996). Ele está localizado em $\alpha(\mathrm{J} 2000)=00^{\mathrm{h}} 24^{\mathrm{m}} 05.359^{\mathrm{s}}, \delta(\mathrm{J} 2000)=-72^{\circ} 04^{\prime} 5320$, suas coordenadas galácticas estão indicadas na tabela 1.1. com distâncias da literatura entre $4.5 \pm 0.45$ ( (Harris, 1996), edição de 2010; (Bica et al., 2006), (Baumgardt e Hilker, 2018)), e 5.10 kpc ((Colaboração Gaia, 2018) baseado no inverso da sua paralaxe (1/plx). A Figura 1.1 mostra uma imagem do 47 Tuc.

Pelos motivos previamente citados, o 47 Tuc é um dos aglomerados mais exaustivamente estudados da Galáxia. Um dos primeiros trabalhos onde esse objeto é citado o de (Shapley, 1918), que consta como o primeiro a fazer medidas de magnitudes e distâncias de aglomerados globulares, sendo o 47 Tuc um entre os 69 analisados por ele. O 47 Tuc também é um aglomerado interessante pois sua posição, cinemática e metalicidade vêm sendo interpretadas de diferentes formas com o passar dos anos. Por exemplo, Armandroff, 1989) o classificou como pertencente ao disco. (Bica et al., 2016) incluiu 47 Tuc em uma categoria de aglomerados "intrusos"e possíveis membros perdidos do bojo, estando em uma categoria de aglomerados com $[\mathrm{Fe} / \mathrm{H}]>-1.0$ e d。> $>4.5 \mathrm{kpc}$. (Barbuy et al., 2018) e Ortolani et al. 1995 consideraram 47 Tuc como um aglomerado pertencente ao halo interno da Galáxia. Mais recentemente, (Pérez-Villegas et al., 2019) calculou as órbitas do aglomerado baseadas em movimentos próprios obtidos da (Colaboração Gaia, 2018), onde foram calculadas as distâncias mínimas e máximas em relação ao Centro Galáctico, cujos resultados foram de 7.2 e 9.4 kiloparsecs $(\mathrm{kpc})$ respectivamente; a altura relativa ao Plano Galáctico $(4,5 \mathrm{kpc})$, e a excentricidade da órbita (0.13), para uma velocidade de rotação 


\begin{tabular}{|cccccccc|}
\hline Aglomerado & $\mathrm{l}(\mathrm{\circ})$ & $\mathrm{b}(\mathrm{\circ})$ & $\mathrm{E}(\mathrm{B}-\mathrm{V})$ & $\begin{array}{c}d_{\odot} \\
(\mathrm{kpc})\end{array}$ & $\begin{array}{c}d_{G C} \\
(\mathrm{kpc})\end{array}$ & $\mathrm{M}_{\mathrm{V}}$ & $\mathrm{N}_{1} / \mathrm{N}_{T O T}$ \\
\hline NGC104 & 305.89 & -44.89 & 0.04 & 4.5 & 7.4 & 14.06 & 0.175 \\
\hline
\end{tabular}

Tabela 1.1 - Coordenadas galácticas, extinção E(B-V), distância ao Sol e ao centro galáctico, magnitude absoluta visual extraído de Harris (1996), e fração de estrelas de primeira geração extraído de Milone et al. (2017)

da barra de $40 \mathrm{~km} \mathrm{~s}^{-1} \mathrm{kpc}^{-1}$, fez com que concluíssem que se trata de um aglomerado pertencente ao halo interno.

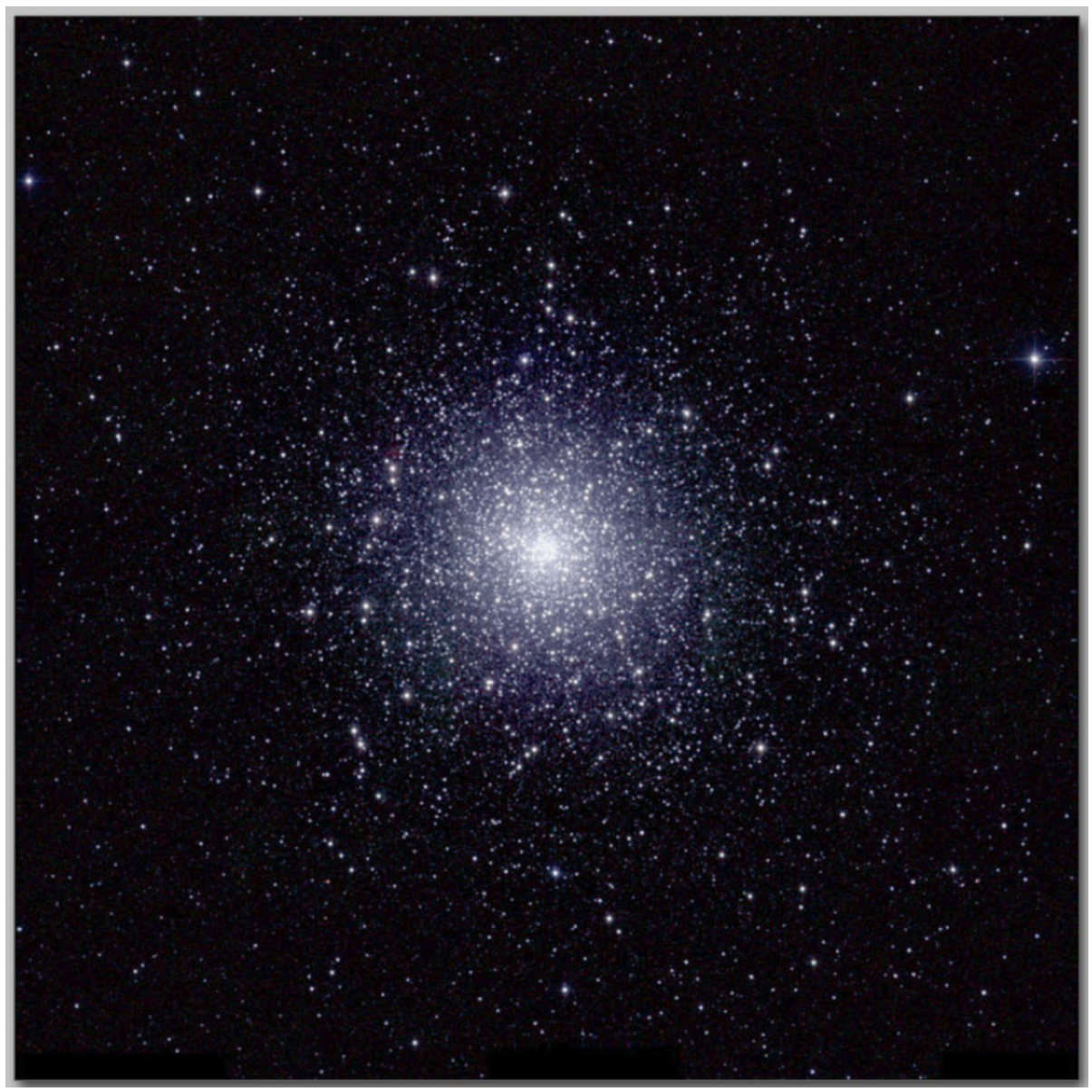

Figura 1.1: Imagem do 47 Tuc. Extraído da página eletrônica da colaboração 2MASS.

\subsubsection{Estudos de Abundâncias do 47 Tuc}

Estudos espectroscópicos de estrelas individuais do 47 Tuc vêm sendo realizados desde o final dos anos 1970, como nos trabalhos de Dickens et al. (1979), Norris e Cottrell (1979) e Pilachowski et al. (1980), que já continham os primeiros esforços em se medir as 
abundâncias químicas de elementos no aglomerado em questão, com foco especialmente no estudo das bandas de $\mathrm{CH}$ e $\mathrm{CN}$. Mais recentemente, análises de espectros de alta resolução de estrelas individuais desse aglomerado forneceram medidas mais precisas e de mais elementos químicos, como expostas nos trabalhos de, Alves-Brito et al. (2005), Koch e McWilliam (2008), Carretta et al. (2009), Thygesen et al. (2014) e Cordero et al. (2014) , onde os resultados indicam que a metalicidade desse GC está no intervalo -0.66 < $[\mathrm{Fe} / \mathrm{H}]<-0.79$, resultando num valor médio de $[\mathrm{Fe} / \mathrm{H}] \sim-0.75$. Apenas mais recentemente espectros integrados de alta resolução do 47 Tuc foram observados, o que possibilitou qualidade suficiente para análises de qualidade equivalente aos estudos de abundâncias químicas derivadas de estrelas individuais. A análise desses espectros foi feita por meio da aplicação de diferentes técnicas.

Graves e Schiavon (2008) utilizaram o código EZ_Ages, em conjunto com isócronas do grupo de Padova, sendo elas de dois tipos: um com abundâncias solares Girardi et al. 2000), e um outro com enriquecimento de elementos $\alpha$ de $[\alpha / \mathrm{Fe}]=0.42$ (Salasnich et al. 2000), além de abundâncias iniciais $[\mathrm{Mg} / \mathrm{Fe}],[\mathrm{C} / \mathrm{Fe}],[\mathrm{N} / \mathrm{Fe}],[\mathrm{O} / \mathrm{Fe}],[\mathrm{Ca} / \mathrm{Fe}],[\mathrm{Na} / \mathrm{Fe}]$, $[\mathrm{Si} / \mathrm{Fe}],[\mathrm{Cr} / \mathrm{Fe}]$, e [Ti/Fe], foram utilizadas para modelar espectros sintéticos nas regiões dos índices de Lick, com diferentes valores de idades e de metalicidades $[\mathrm{Fe} / \mathrm{H}]$. Esses índices teóricos foram comparados com os índices de espectros integrados observados. As idades e o valor de $[\mathrm{Fe} / \mathrm{H}]$ para os espectros sintéticos foram obtidos de forma automática pelo programa, utilizando linhas que são fortemente afetadas pelas variações desses dois parâmetros, porém insensíveis a variações de abundâncias de outros elementos químicos. O código comporta idades dentro do intervalo $0.1 \leqslant \mathrm{t} \leqslant 15.8$ Giga Anos (abreviado como Gyr), metalicidades entre $-1.3 \leqslant[\mathrm{Fe} / \mathrm{H}] \leqslant+0.2$ para o caso das isócronas solares e $-0.8 \leqslant$ $[\mathrm{Fe} / \mathrm{H}] \leqslant+0.3$ para as com enriquecimento de elementos $\alpha$. Os índices de Lick utilizados foram $\mathrm{H} \delta_{A}, \mathrm{H} \delta_{F}, \mathrm{H} \gamma_{A}, \mathrm{H} \gamma_{F}, \mathrm{H} \beta, \mathrm{CN} 1, \mathrm{CN} 2, \mathrm{Ca} 4227, \mathrm{G} 4300, \mathrm{Fe} 4383, \mathrm{C} 24668, \mathrm{Fe} 5015$, $\mathrm{Mg} 2, \mathrm{Mg}$ b, Fe5270, and Fe5335. Os índices calculados puderam então ser comparados com aqueles medidos em espectros observados, para então se determinar qual era a melhor idade e metalicidade para a o objeto observado, correspondendo a um determinado grupo de abundâncias elementais. A amostra de aglomerados inclui o 47 Tuc, e os resultados obtidos para esse objeto estão na tabela 1.2 .

McWilliam e Bernstein (2008) e Colucci et al. (2017) utilizaram medidas de larguras equivalentes (daqui em diante citadas como EW, do inglês Equivalent Widths) e diagra- 
mas cor-magnitude (daqui pra frente citados como CMDs, Color Magnitude Diagrams, do inglês) empirícos para a construção dos espectros sintéticos, e para a investigação das abundâncias elementais do 47 Tuc. A técnica empregada consistiu em dividir o CMD em múltiplas regiões com diferentes tipos de estrelas, e assim calcular EWs para cada uma das linhas de sua lista, em cada uma dessas regiões demarcadas do CMD. A EW final para cada linha foi calculada a partir de uma combinação dos fluxos de cada uma das regiões do CMD, considerando seu respectivo peso estatístico. As abundâncias foram calculadas por meio de ajustes entre as EW observados e as calculadas de forma iterativa, até que a calculado coincida com a observada em luz integrada. Para a medida das EWs dos espectros observados, foi utilizado o código GETJOB (McWilliam et al., 1995). Para a obtenção do melhor ajuste, também foi utilizado o pacote ILABUNDS, que utiliza modelos de atmosferas estelares com enriquecimento de elementos $\alpha$ de Kurúcz Castelli e Kurucz, 2004) e o código MOOG (Sneden, 1973) para a síntese espectral. Sua lista inclui linhas de Na, Mg, Al, Si, Ca, Sc, Ti, V, Cr, Mn, Fe, Co, Ni, Cu, Y, Zr, Ba, La, Nd, e Eu no intervalo de $5000-7570 \AA$.

Colucci et al. (2017) analisou o 47 Tuc utilizando as mesmas técnicas previamente aplicadas em McWilliam e Bernstein (2008), porém introduzindo mudanças significativas: Foram utilizadas isócronas teóricas BaSTI do grupo de Teramo (Pietrinferni et al., 2004, 2006) para o CMD, e uma função de massa inicial (daqui adiante citada como IMF, do inglês Inital Mass Function) de Kroupa (2001). De forma similar à análise prévia realizada em McWilliam e Bernstein (2008), atmosferas em 1D, plano-paralelas, e que consideram condições de equilíbrio termodinâmico local (adiante citado como LTE, do inglês Local Thermodynamic Equilibrium) foram utilizadas (Castelli e Kurucz, 2004), assim como o pacote ILABUNDS, para a criação do espectro sintético e para as medidas das EWs. A lista de linhas utilizada inclui linhas de Na I, Mg I, Al I, Si I, Ca I, Ti I, Ti II, Sc II, V I, Cr I, Mn I, Co I, Ni I, Cu I, Y II, Zr I, Ba II, La II, Nd II, e Eu II. Algumas das linhas utilizadas para medidas de abundância por eles também foram utilizadas neste trabalho, e uma comparação entre os resultados será mostrada na tabela 3.2 .

Larsen et al. (2017) utilizou a técnica de Full Spectrum Fitting para a análise de espectros integrados de sua amostra de aglomerados globulares, incluindo o 47 Tuc. Essa técnica consiste em realizar um ajuste simultâneo de múltiplas linhas, ou seja, do espectro todo, de modo a encontrar o melhor ajuste entre os espectros observado e sintético, e assim 
calcular o melhor conjunto de abundâncias elementais que satisfaz essa condição. Nesse trabalho, foram utilizados espectros integrados de alta resolução obtidos com o UVES do ESO $(\mathrm{R} \sim 40,000)$, e para encontrar o melhor ajuste entre os espectros, as abundâncias utilizadas para a geração do espectro sintético foram ajustadas de forma iterativa, até que o melhor valor médio para o ajuste de todas as linhas fosse atingido. Foram adotadas isócronas teóricas e também CMDs empíricos, que foram divididos em regiões (ou bins), como nos trabalhos anteriormente citados. Para os CMDs empíricos, foram utilizados dados fotométricos da Advanced Camera for Surveys do Telescópio Espacial Hubble (Sarajedini et al., 2007; Anderson et al., 2008). As isócronas teóricas utilizadas consideram enriquecimento de elementos $\alpha$ do grupo de Dartmouth (Dotter et al., 2008), e foram utilizadas apenas para os bins menos luminosos. Em sua análise, eles concluíram que a aplicação de isócronas teóricas ou CMDs empíricos produzem resultados similares nos espectros sintéticos criados. Para a construção dos espectros sintéticos, foi considerada uma IMF de Salpeter (Salpeter, 1955), atmosferas estelares teóricas MARCS (Gustafsson et al., 2008) e o código TURBOSPECTRUM (Alvarez e Plez, 1998; Plez, 2012) para a sintese espectral.

Conroy et al. (2018) também utilizam "full spectral fitting"para medir abundâncias elementais no 47 Tuc e outros 40 aglomerados. Para isso, são utilizadas isócronas do grupo de Dartmouth, IMF de Kroupa (2001) e as bibliotecas de espectros estelares MILES (Sánchez-Blázquez et al., 2006), combinadas com a biblioteca espectral empírica de IRTF estendida (Prugniel et al., 2011; Sharma et al., 2016), que são ainda complementadas com uma biblioteca de anãs M (Mann et al., 2015). Um polinômio interpolador espectral associa cada ponto da isócrona a um espectro estelar adequado pertencente a uma das bibliotecas. Como esse tipo de biblioteca é limitado quanto a variações de abundâncias, devido ao fato de que são constituídas de espectros estelares empíricos, foram desenvolvidas funções resposta para atenuar esse problema. Os espectros integrados sintéticos desenvolvidos por eles são comparados com espectros integrados empíricos publicados em Schiavon et al. (2005) e abundâncias de Mg, Si, Ca, and Ti foram obtidas.

Na tabela 1.2 estão as abundâncias da literatura para o 47 Tuc, assim como o método utilizado para sua obtenção. Os estudos estão divididos em categorias, sendo de espectroscopia de alta resolução (HRS) de estrelas RGBs e de gigantes vermelhas do ramo assintótico (adiante citadas como AGBs, de Assymptotic Giant Branch), e oriundas de análises de es- 
Tabela 1.2 - Metalicidades e abundâncias da literatura para o 47 Tuc. Os dados apresentados são divididos por tipo de análise, resolução e tipo de objeto observado para a análise, onde são divididos em espectros de alta resolução (HRS), espectros integrados e espectros de média resolução (MRS). A última linha contém a compilação feita em Roediger et al. (2014). Referências: 1 - (Koch e McWilliam, 2008)) com dados do espectrógrafo Echelle de alta resolução Magellan Inamori Kyocera instalado no telescópio Magellan 6.5 m Clay; 2 - (Alves-Brito et al., 2005) com dados do UVES do VLT-ESO; 3 - Cordero et al. 2014) com dados do espectrógrafo multi-fibra Hydra instalado no telescópio Blanco de 4m, e com dados do FLAMES do VLT-ESO; 4 - (Thygesen et al., 2014) com dados do UVES do VLT-ESO; 5 - Carretta et al. 2009) com dados em sua maioria do UVES do VLT-ESO; 6 - (Pancino et al., 2017) com dados do Gaia-ESO survey, obtidos com o UVES e com o GIRAFFE do VLT; 7 - (Wylie et al. 2006) com dados de AGBs observadas com o espectrógrafo echelle UCLES do telescópio Anglo-Australiano; 8 - Colucci et al. 2017) com dados do telescópio Du Pont de Las Campanas; 9 - (McWilliam e Bernstein, 2008) com dados do telescópio Du Pont em Las Campanas; 10 - (Larsen et al., 2017) com dados do UVES do VLT-ESO; 11 - (Graves e Schiavon, 2008) com dados de (Schiavon et al. 2005), que utilizou o espectrógrafo R-C do telescópio Blanco do observatório Cerro Tololo; 12 - (Conroy et al., 2018) com os mesmos dados de (Schiavon et al., 2005); 13 - Compilação realizada por (Roediger et al., 2014).

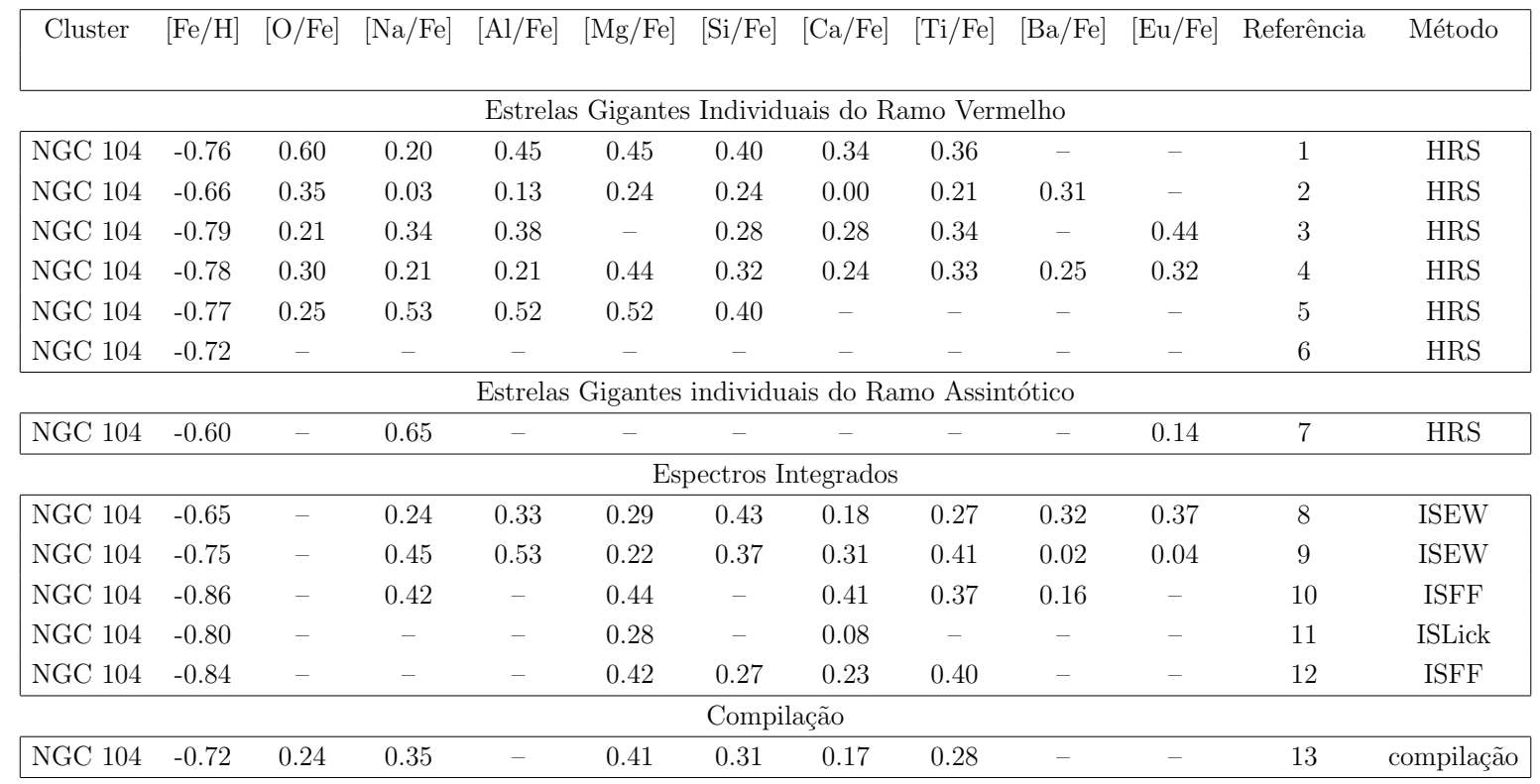

pectros integrados por meio de: índices de Lick (ISLick), medidas de larguras equivalentes (ISEW) e full spectrum fitting (ISFF). Os últimos valores da tabela são oriundos de uma compilação feita por (Roediger et al., 2014), que inclui a maioria dos resultados previamente calculados e apresenta uma média dos valores de abundâncias obtidos para o 47 Tuc.

\subsubsection{Objetivos}

O 47 Tuc é um dos aglomerados globulares mais exaustivamente estudados, e por esse motivo, é um excelente objeto para a aplicação de novos métodos e técnicas, dada 
a vasta gama de resultados já previamente obtidos. O objetivo desse trabalho é estudar, através de espectros integrados, o espectro do 47 Tuc recentemente publicado, obtido com o espectrógrafo WiFes (integral field unit Wide-Field spectrograph) instalado no telescópio de $2.3 \mathrm{~m}$ da Australian National University, e apresentado em (Usher et al., 2017), contido no WiFeS Atlas of Galactic Globular Cluster Spectra (WAGGS). Para tal, foi utilizado o pacote SynSSP, criado por Marina Trevisan, e posteriormente utilizado e aprimorado por nosso grupo, previamente aplicado em (La Barbera et al., 2013) e apresentado em (Moura et al. 2019), que será descrito em detalhes a seguir, assim como os resultados da análise realizada. 
Capítulo 2

\section{Metodologia}

\subsection{Dados Observacionais}

O espectro integrado observado foi extraído do survey WAGGS (WiFeS Atlas of Galactic Globular cluster Spectra), apresentado em Usher et al. (2017). Esse survey apresenta uma biblioteca de 64 aglomerados globulares da Via Láctea, além de 22 outros aglomerados localizados nas galáxias anãs de Fornax e nas Grande e Pequena Núvens de Magalhães. Esses espectros foram coletados com o espectrógrafo de campo integral WiFeS, instalado no telescópio de 2.3 metros da Australian National University, e abrangem o intervalo de comprimento de onda 3270 - $9050 \AA$, com uma resolução espectral de $R=6800$, que é superior às resoluções das bibliotecas espectrais previamente disponíveis para esse tipo de objeto (como por exemplo, as de Schiavon et al., 2005; Puzia et al., 2002). Os espectros disponibilizados já foram previamente reduzidos pela própria pipeline do telescópio em questão. A amostra de GCs abrange também objetos velhos e novos, contendo GCs com idades entre 20 milhões de anos (Myr) e 13 bilhões de anos (Gyr).

\subsection{Espectros Sintéticos}

Para se modelar o espectro de um GC, que é tratado como uma população única de estrelas (Single Stellar Population, simplesmente SSP), são necessárias trajetórias evolutivas estelares, assim como sua respectiva biblioteca de espectros refentes às estrelas, como exemplificado em Martins et al. (2019). Ambos os ingredientes podem ser compostos de dados empíricos ou teóricos. Para a formação do espectro integrado referente a uma SSP, o espectro de diferentes estrelas em diferentes estágios evolutivos são combinados, com seus respectivos pesos estatísticos, de acordo com uma IMF determinada. 
As isócronas (ou no caso empírico, CMDs) determinam as massas, luminosidades e parâmetros atmosféricos (que são a temperatura efetiva $\mathrm{T}_{\text {eff }}$ e gravidade superficial log $g$ ) das estrelas cujos espectros serão combinados para a formação do espectro integrado, dados a idade, metalicidade e $[\alpha / \mathrm{Fe}]$ da população. Esses espectros são selecionados pelas quadras de $\mathrm{T}_{e f f}, \log g, \mathrm{~m}$ e $\log \left[\mathrm{L} / \mathrm{L}_{\odot}\right]$, e podem ser oriundos de bibliotecas teóricas ou empíricas. Como neste trabalho, as linhas são ajustadas individualmente, bibliotecas empíricas não são adequadas, por estarem restritas aos padrões de abundâncias elementais da vizinhança solar.

Para a confecção dos espectros sintéticos, foi desenvolvido o pacote SynSSP, que permite o cálculo de SSPs com abundâncias variáveis. O programa funciona com três rotinas básicas, onde uma seleciona as estrelas na isócrona (stpars), outra calcula os espectros estelares referentes a cada um dos grupos de dados $\mathrm{T}_{\text {eff }}-\log g-\mathrm{M}-\log \left[\mathrm{L} / \mathrm{L}_{\odot}\right]$ selecionados previamente (pfant12) e a última combina esses espectros para criar o espectro referente à SSP desejada (ssp.model). Um detalhamento das funções utilizadas pelo programa, baseado no manual de instruções que consta no SynSSP está a seguir.

As seguintes entradas são necessárias para a rotina stpars:

- Idade, metalicidade $[\mathrm{Fe} / \mathrm{H}]$, e $[\alpha / \mathrm{Fe}]$ da população. As isócronas utilizadas são as do grupo de Dartmouth (Dotter et al. (2008)), já amplamente utilizadas pelo nosso grupo;

- Número de estrelas a serem consideradas na isócrona. O procedimento padrão leva em consideração 12 estrelas na sequência principal e ramo das subgigantes, e 9 estrelas no ramo das gigantes vermelhas, que se mostraram um número suficiente para a obtenção de um espectro sintético próximo ao observado em Usher et al. (2017).

Após selecionados as quadras $\mathrm{T}_{\text {eff }}-\log g-\mathrm{M}-\log \left[\mathrm{L} / \mathrm{L}_{\odot}\right]$, são necessários os seguintes parâmetros para a rotina pfant12:

- Valores das abundâncias de [C, N, O, Na, Mg, Si, Ca, Ti, Ba, Eu/Fe]. Os valores padrão são de $[\mathrm{C} / \mathrm{Fe}]=-0.2,[\mathrm{~N} / \mathrm{Fe}]=+1.0$ e de $[\alpha / \mathrm{Fe}]$ para os elementos $[\mathrm{O}, \mathrm{Mg}, \mathrm{Si}, \mathrm{Ca}, \mathrm{Ti}$, Eu/Fe]. Para outros elementos, o padrão é o da abundância solar encontrada em Asplund et al. (2009), porém, esses valores podem ser modificados pelo usuário, caso desejado. 


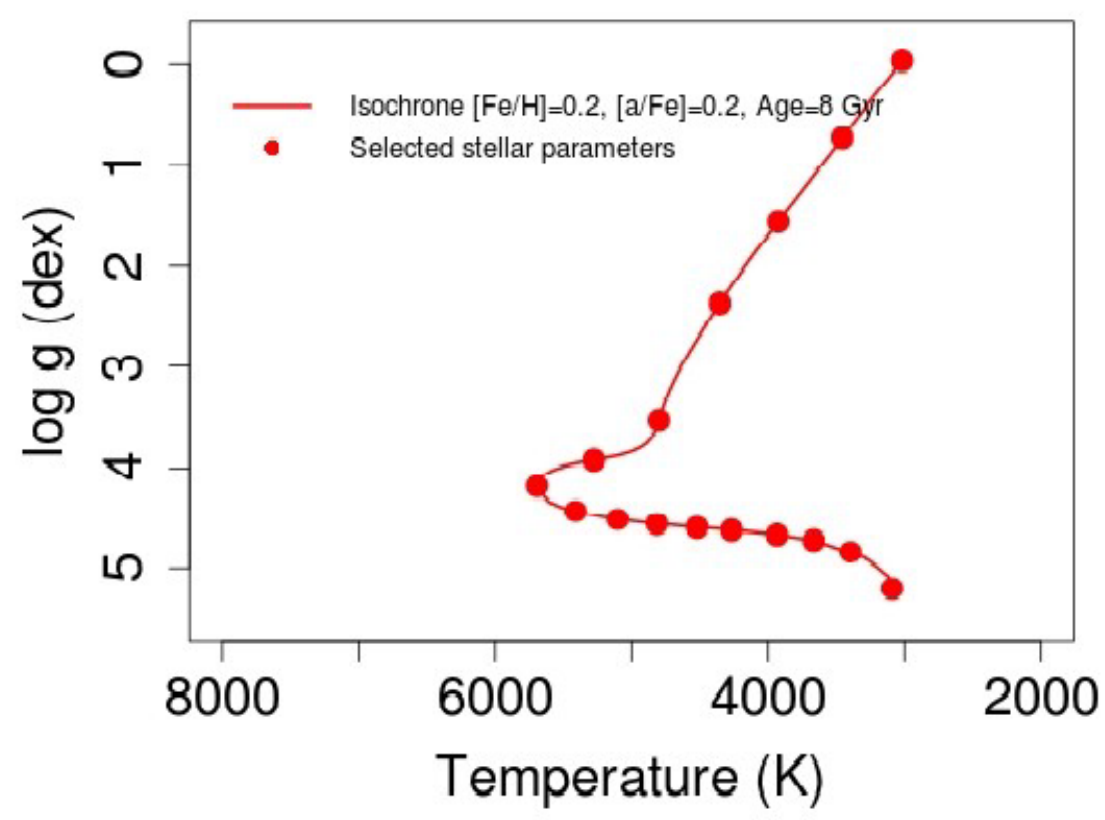

Figura 2.1: Exemplo de isócrona criada pela função stpars.

- Amostragem $(\delta \lambda)$, largura máxima a meia altura (Full Width at the Half Maximum, FWHM) e intervalo de comprimento de onda a ser gerado. Os valores padrão para as resoluções são de FWHM $=0.2 \AA$ e $\delta \lambda=0.12 \AA$.

Os espectros sintéticos são calculados pelo programa PFANT, recentemente atualizado e descrito em detalhes em Barbuy et al. (2018). O programa calcula, a partir de uma lista de linhas atômicas e moleculares, além de uma dada atmosfera estelar, espectros estelares, considerando condições de LTE. A lista de linhas atômicas utilizada é a VALD3 (Ryabchikova et al. (2015)), porém, para algumas linhas, especialmente as dos tripletos

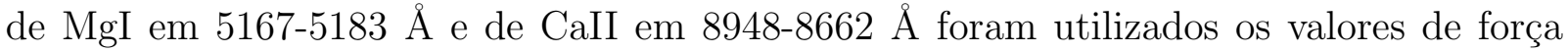
de oscilador mais atualizados, apresentados em Pehlivan Rhodin et al. (2017) e Kurucz (2005a) (ver tabela 2.1). A lista de linhas moleculares adotada foi a mesma de Barbuy et al. (2018). As atmosferas estelares adotadas são as do modelo MARCS em condições LTE (Gustafsson et al. (2008)), que já foram utilizadas anteriormente em trabalhos do nosso grupo. Para cada um dos grupos $\mathrm{T}_{\text {eff }}-\log g-\mathrm{M}-\log \left[\mathrm{L} / \mathrm{L}_{\odot}\right]$ da isócrona, um espectro estelar é calculado, e esses espectros são combinados por meio da rotina ssp.model. Essa rotina lê os valores de $\mathrm{T}_{\text {eff }}-\log g-\mathrm{M}-\log \left[\mathrm{L} / \mathrm{L}_{\odot}\right]$ assim como os espectros $\mathrm{S}(\operatorname{lambda}, M)$ associados a cada um desses grupos de dados obtidos nos passos anteriores e, dada uma 
IMF, divide as estrelas teóricas em $\mathrm{N}$ grupos (ou bins), da seguinte forma:

Bin 1: massas a partir de $\mathrm{M}_{l, 1}=0.08 \mathrm{M}_{\odot}$

até $\mathrm{M}_{u, 1}=\mathrm{M}\left[\mathrm{L}=\left(\mathrm{L}_{2}+\mathrm{L}_{1}\right) / 2\right]$;

Bin i: massas a partir de $\mathrm{M}_{l, i}=\mathrm{M}\left[\mathrm{L}=\left(\mathrm{L}_{i-1}+\mathrm{L}_{i}\right) / 2\right]$

até $\mathrm{M}_{u, i}=\mathrm{M}\left[\mathrm{L}=\left(\mathrm{L}_{i}+\mathrm{L}_{i+1}\right) / 2\right]$;

Bin N: massas a partir de $\mathrm{M}_{l, N}=\mathrm{M}\left[\mathrm{L}=\left(\mathrm{L}_{N-1}+\mathrm{L}_{N}\right) / 2\right]$

até $\left.\mathrm{M}_{u, N}=\mathrm{M}_{N}\right]$;

Onde $\mathrm{M}_{L}$ corresponde a massa estelar de uma estrela com luminosidade $\mathrm{L}$ (que é interpolada a partir da isócrona utilizada). É importante notar que as massas estão distribuídas de forma crescente, ou seja, $M_{i}<M_{i+1}$. A partir dessa divisão, são realizados os seguintes cálculos para cada um desses bins:

$$
\begin{gathered}
M_{b i n}=\int_{M_{l}}^{M_{u}} m \Phi(m) d m \\
L_{b i n}=\int_{M_{l}}^{M_{u}} L(m) \Phi(m) d m \\
L_{\text {star }}=L_{i} \int_{M_{l}}^{M_{u}} \Phi(m) d m \\
N_{\text {bin }}=\int_{M_{l}}^{M_{u}} \Phi(m) d m \\
L_{\text {corr }}=L_{\text {bin }} / L_{\text {star }}
\end{gathered}
$$


Onde $M_{l}$ e $M_{u}$ são os limites inferiores e superiores da massa do bin, $\Phi(m)$ é a IMF, (2.1) é a massa total estelar do bin, (2.2) é a luminosidade total do bin, (2.3) é a luminosidade total do bin considerando que $L(m)=$ constante $=L_{i},(2.4)$ é o número de estrelas no bin e (2.5) é um fator de correção com fim de levar em consideração a variação de luminosidade dentro do bin. Com esses resultados, o espectro integrado da SSP pode ser calculado através da equação,

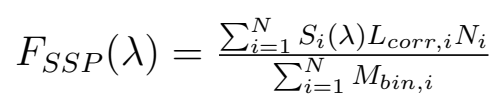

Onde $S_{i}(\lambda)$ é o espectro da estrela. Os limites das integrais são $\mathrm{M}_{l}=0.08 \mathrm{M}_{\odot}$ e $\mathrm{M}_{u}$ é a maior massa estelar da isócrona utilizada nos passos anteriores.

\subsection{Valores Utilizados para o 47 Tuc}

Como anteriormente exposto, uma série de parâmetros precisam ser fornecidos para que o SynSSP possa criar uma SSP teórica de um determinado espectro. Para o presente trabalho, após uma série de testes com os valores da literatura apresentados na tabela 1.2 , foi encontrado que o valor para a metalicidade que melhor se ajustou ao espectro de Usher et al. (2017) foi de $[\mathrm{Fe} / \mathrm{H}]=-0.79$, dado por Thygesen et al. (2014), assim como o valor de $[\alpha / \mathrm{Fe}]=+0.4$. Para as isócronas, foi adotado o valor de 11 Gyr (VandenBerg et al. (2013)). Vide a tabela 2.2 para outros valores de idades da literatura para esse objeto.

Tabela 2.1 - Constantes atômicas para os tripletos de MgI e CaII. Referências: 1 - Pehlivan Rhodin et al. (2017), 2 - Kurucz (2005a). Extraído de Moura et al. (2019).

\begin{tabular}{|lcccccc|}
\hline Espécie & $\lambda(\AA)$ & $\chi_{\mathrm{ex}}(\mathrm{eV})$ & $\log \mathrm{gf}$ & $\mathrm{C}_{6}$ & $\gamma_{R} / \mathrm{NH}$ & $\gamma_{e} / \mathrm{NH}$ \\
\hline $\mathrm{MgI}$ & 5167.3216 & 2.7091 & $-0.854^{1}$ & $0.3 \mathrm{E}-29$ & $1.0 \mathrm{E}+08$ & $2.0 \mathrm{E}-07$ \\
$\mathrm{MgI}$ & 5172.684 & 2.7116 & $-0.363^{1}$ & idem & idem & idem \\
$\mathrm{MgI}$ & 5183.604 & 2.7166 & $-0.168^{1}$ & idem & idem & idem \\
CaII & 8498.023 & 1.6924 & $-1.312^{2}$ & $0.9 \mathrm{E}-32$ & $3.6 \mathrm{E}+08$ & $2.6 \mathrm{E}-07$ \\
CaII & 8542.091 & 1.6999 & $-0.362^{2}$ & $0.8 \mathrm{E}-32$ & $3.0 \mathrm{E}+08$ & idem \\
CaII & 8662.141 & 1.6924 & $-0.623^{2}$ & $0.8 \mathrm{E}-32$ & $2.95 \mathrm{E}+08$ & idem \\
\hline
\end{tabular}


Tabela 2.2 - Idades da literatura (Gyr) para o 47 Tuc.

\begin{tabular}{|c|c|c|}
\hline Idade & Método & Referência \\
\hline $11 \mathrm{a} \sim 13$ & Espectroscopia & Schiavon et al. $(2002)$ \\
\hline 11.3 & Ajuste da Sequência Principal & Gratton et al. 2003$)$ \\
\hline 11.25 & Eclipse de Estrelas Binárias (V69) & Thompson et al. (2010) \\
\hline 11.75 & Ajuste da Sequência Principal & VandenBerg et al. (2013) \\
\hline 12 & Ajuste da Sequência Principal & VandenBerg et al. $(2014)$ \\
\hline 13 & Ajuste da Sequência Principal & Denissenkov et al. $(2017)$ \\
\hline 11.8 & Ajuste Sequência Principal e V69 & Brogaard et al. (2017) \\
\hline
\end{tabular}

Foram utilizados os valores de FWHM $=1.0 \AA$ e de $\delta \lambda=0.1 \AA$, para as comparações desejadas com o espectro observado.

Foram adotados valores iniciais para as abundâncias elementais de acordo com Thygesen et al. (2014), mas para o ajuste das linhas, os valores foram alterados de modo a encontrar o valor de abundância que melhor ajusta o espectro sintético à linha de interesse. As linhas ajustadas estão listadas na tabela 3.3. As abundâncias foram obtidas de forma similar a métodos utilizados para a análise de espectros em alta resolução, ou seja, por meio do ajuste do contínuo localmente, e variando a abundância do elemento em questão até que se encontre o melhor ajuste para a linha analisada. 
Capítulo 3

\section{Resultados}

Seguindo os passos explicados na seção anterior, foi calculado o espectro integrado total no intervalo de 4500 - $9000 \AA$ (exibido no Apêndice A). O ajuste para o espectro integrado total foi feito com as abundâncias médias, calculadas após o ajuste das linhas, e apresenta boa coerência com o espectro observado, como pode ser visto nas figuras A.1 a A.5. Também foram calculados ajustes para linhas de elementos de interesse, dos quais foram obtidas as abundâncias elementais, por um procedimento parecido com o da análise de espectros de alta resolução, como explicado anteriormente. Nos casos das linhas fortes (tripletos de $\mathrm{MgI}$ e CaII), apenas as asas das linhas foram ajustadas, uma vez que o fundo dessas linhas é gerado em processos fora do equilíbrio termodinâmico, e não são bem reproduzidas pelos pacotes utilizados.

\subsection{Ajustes de Linhas Individuais}

Foram realizados ajustes de linhas individuais de NaI, MgI, AlI, SiI, CaI, TiI, TiII, BaII e EuII, e os resultados estão listados na tabela 3.3. Os ajustes das linhas de Na foram feitos de forma distinta, devido a sua conexão com estudos de múltiplas populações. Os resultados para o $\mathrm{Na}$ estão na seção que trata de múltiplas populações em aglomerados globulares. Os valores médios obtidos são assumidos como sendo as abundâncias do aglomerado. Foram utilizadas linhas de média intensidade, (evitando linhas muito fortes) para a derivação das abundâncias, pois nesse caso, as EWs variam mais com a abundância (vide Gray (2005)). As abundâncias adotadas para ajuste de cada linha estão indicadas nas figuras em azul. O critério utilizado para o melhor ajuste foi o critério visual, ou seja, o quão bem os pontos observados se ajustam ao espectro sintético produzido. 


\subsubsection{Linhas de $\mathrm{Mg}$}

A Figura 3.1 mostra os ajustes realizados para as linhas em 5528.405 e $8806.756 \AA$ do MgI. Essas linhas indicam uma abundância média de $[\mathrm{Mg} / \mathrm{Fe}]=+0.45$. O tripleto do $\mathrm{MgI}$ localizado em 5167-5183 $\AA$ é bem ajustado por essa abundância, como pode ser visto na Figura 3.12. As forças de oscilador foram atualizadas com os resultados de Pehlivan Rhodin et al. (2017) e o ajuste das asas foi feito de acordo com Moura et al. (2019) (ver tabela 2.1). O fundo das linhas fortes não é bem ajustado pois se forma nas camadas cromosféricas das estrelas, em condições não-LTE, que não são incluídas nos modelos fotosféricos. Os resultados estão em bom acordo com o espectro observado.
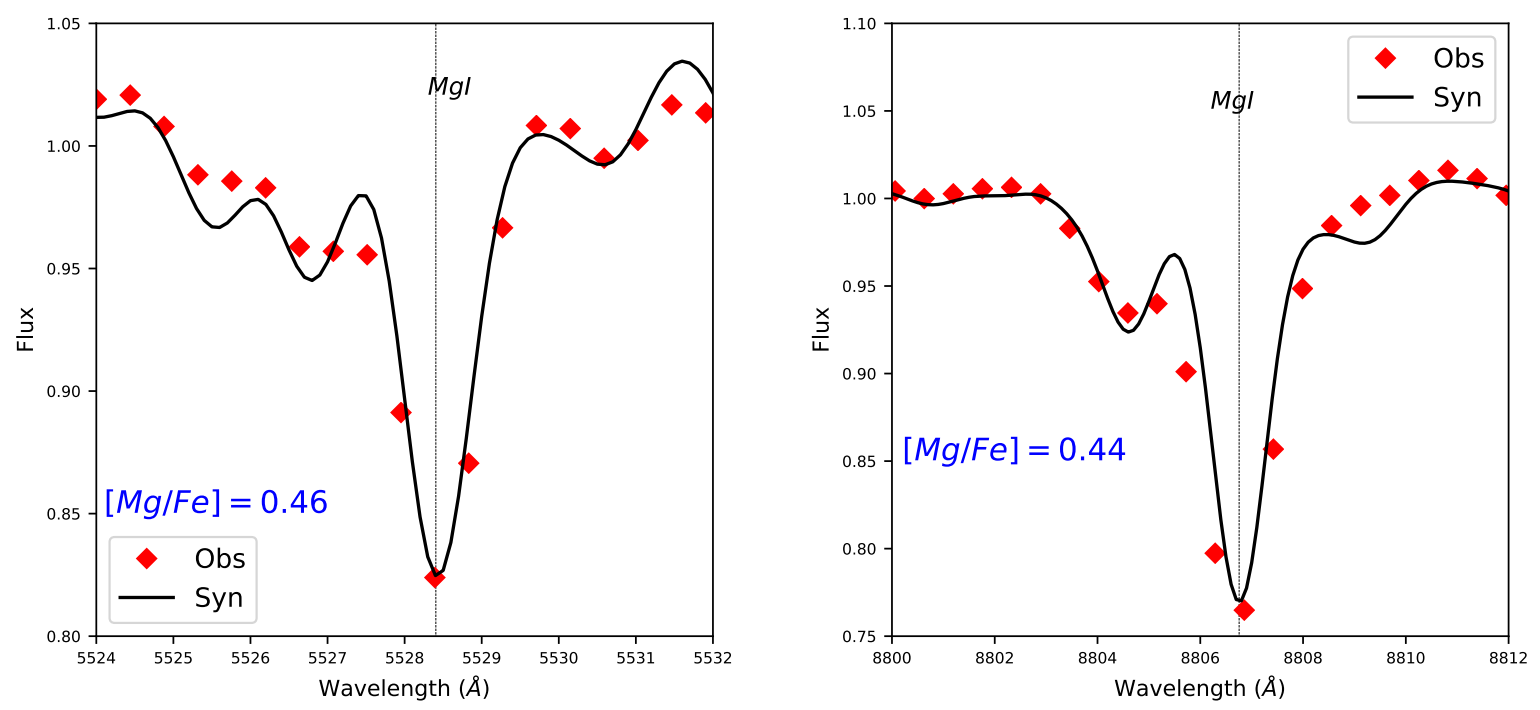

Figura 3.1: Espectros observado e sintético para as linhas de MgI em 5528.405 e 8806.756 A, respectivamente.

\subsubsection{Linhas de Al}

As linhas de AlI em 6696.185, 6696.788 e $6698.673 \AA$ mostradas na Figura 3.2 indicam uma abundância média de $[\mathrm{Al} / \mathrm{Fe}]=+0.24$. É importante ressaltar que excessos de $\mathrm{Al}$ também podem ser indicadores da presença de uma segunda população estelar em GCs (vide Bastian e Lardo (2018), Gratton et al. (2004) e referências citadas). Os valores de abundância satisfazem bem o espectro observado. 

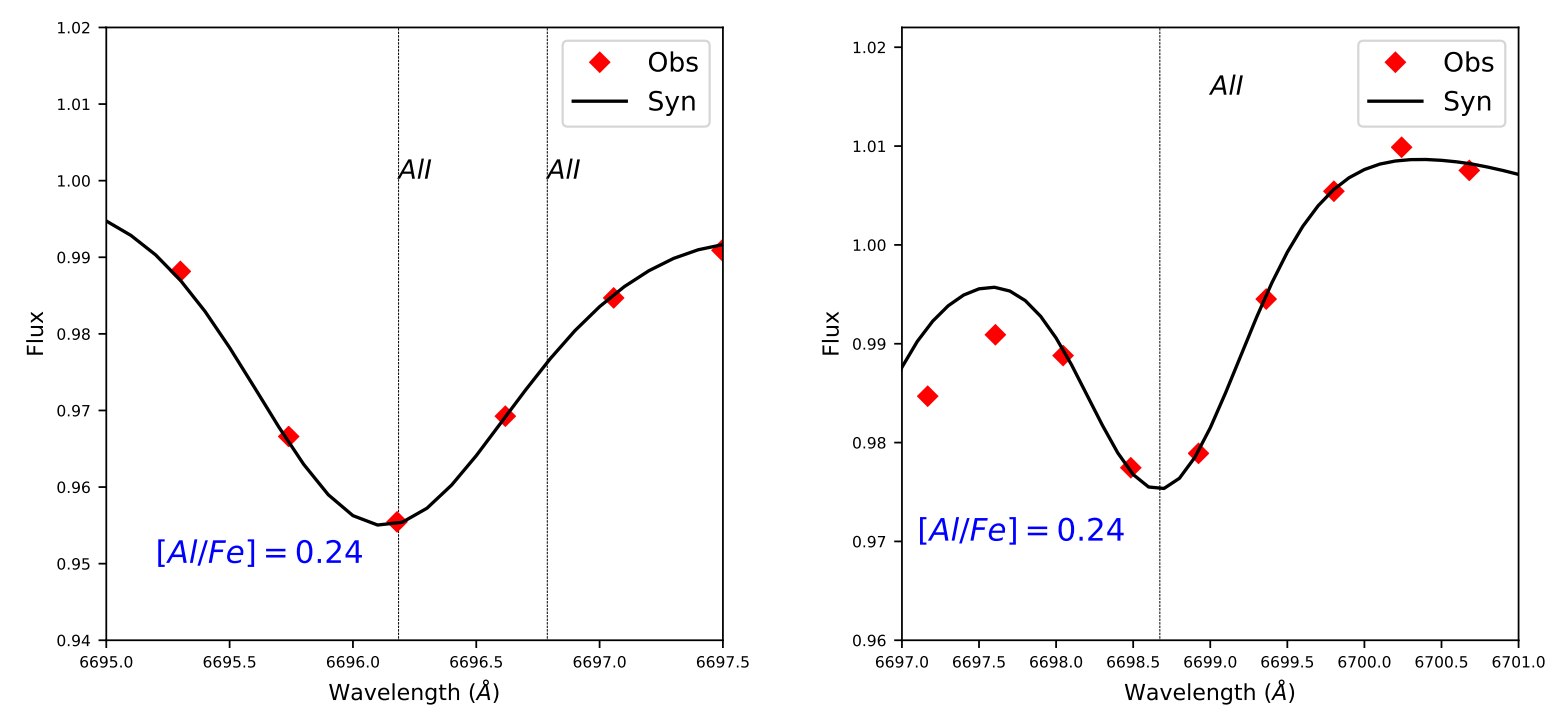

Figura 3.2: Espectros observado e sintético para as linhas de AlI em 6696.185, 6696.788 e $6698.673 \AA$, respectivamente.

\subsubsection{Linhas do Si}

Os ajustes realizados para as linhas de SiI localizadas em 5948.55, 7405.79, 7415.96 e 7423.51 Å estão apresentados na Figura 3.3 , e indicam um valor médio para a abundância de $\mathrm{Si}$ de $[\mathrm{Si} / \mathrm{Fe}]=+0.30$.

\subsubsection{Linhas do Ca}

As linhas ajustadas de CaI em 6102.723, 6122.217 , 6161.295, 6162.167, 6166.44 e 6169.044 e $6169.56 \AA$ A além da linha de NaI em $6160.753 \AA$ estão apresentadas na Figura 3.4. As linhas de CaI em 6439.08, 6455.605, 6717.687 A estão apresentadas na Figura 3.5. O valor médio para a abundância de Ca encontrado é de $[\mathrm{Ca} / \mathrm{Fe}]=+0.20$. Esse valor é igual ao valor de $[\mathrm{Ca} / \mathrm{Fe}]=+0.20$, obtido em Usher et al. (2019) por meio de medidas de EWs das linhas do tripleto de CaII localizado em 8498 - $8662 \AA$. É interessante notar que, embora tenham sido usados tipos de linhas diferentes (com linhas médias e fracas neste trabalho, e linhas fortes em Usher et al. (2019)), os resultados obtidos são semelhantes.

\subsubsection{Linhas do Ti}

As linhas de TiI localizadas em 5866.449, 5941.75, 5965.825 e 5978.539 A estão apresentadas na figura 3.6, as localizadas em 6126.214, 6261.106, 6266.01 e 6556.077 A estão 

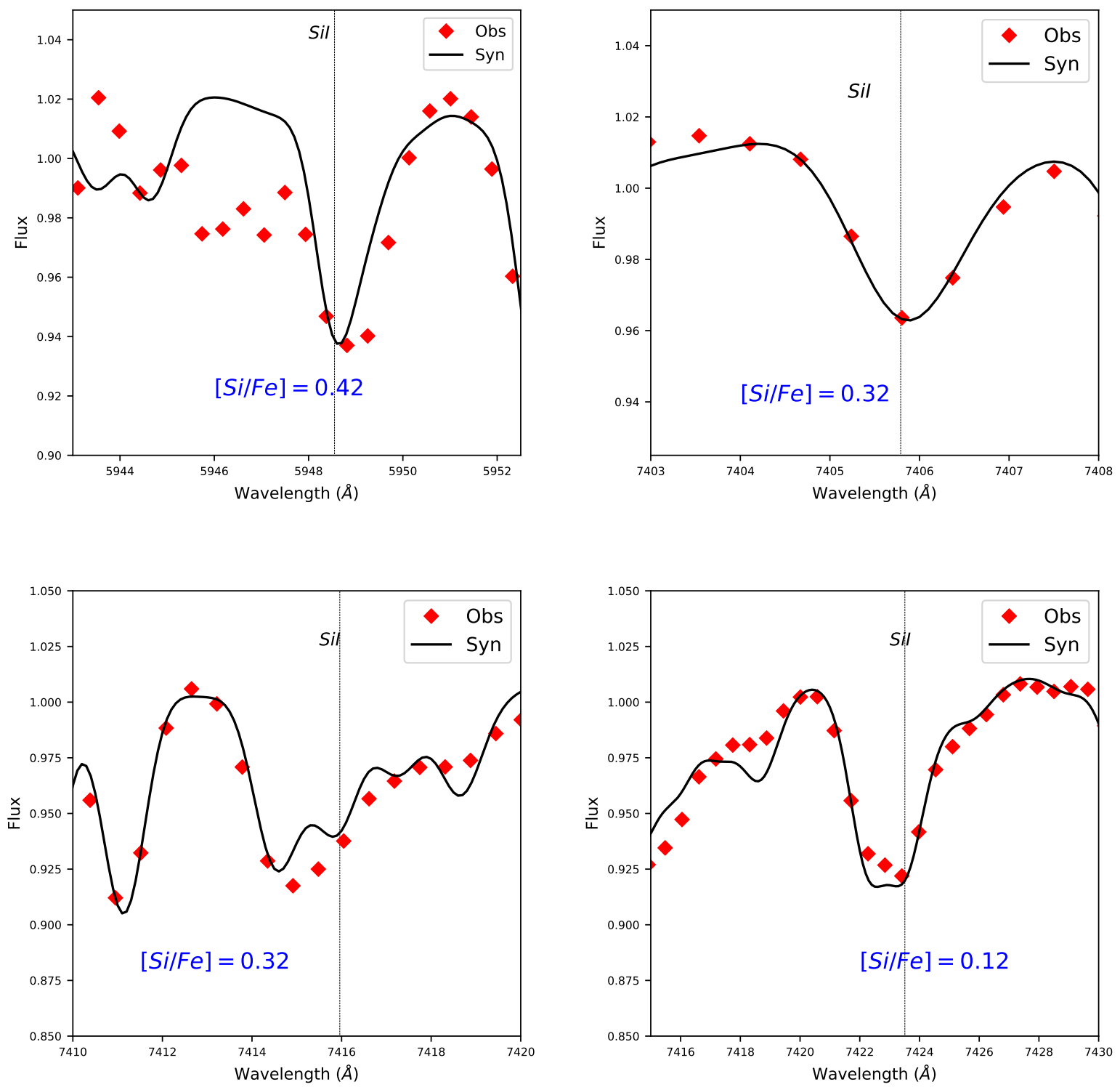

Figura 3.3: Espectros observado e sintético para as linhas de SiI em 5948.55, 7405.79, 7415.96 e 7423.51 $\AA$, respectivamente. 

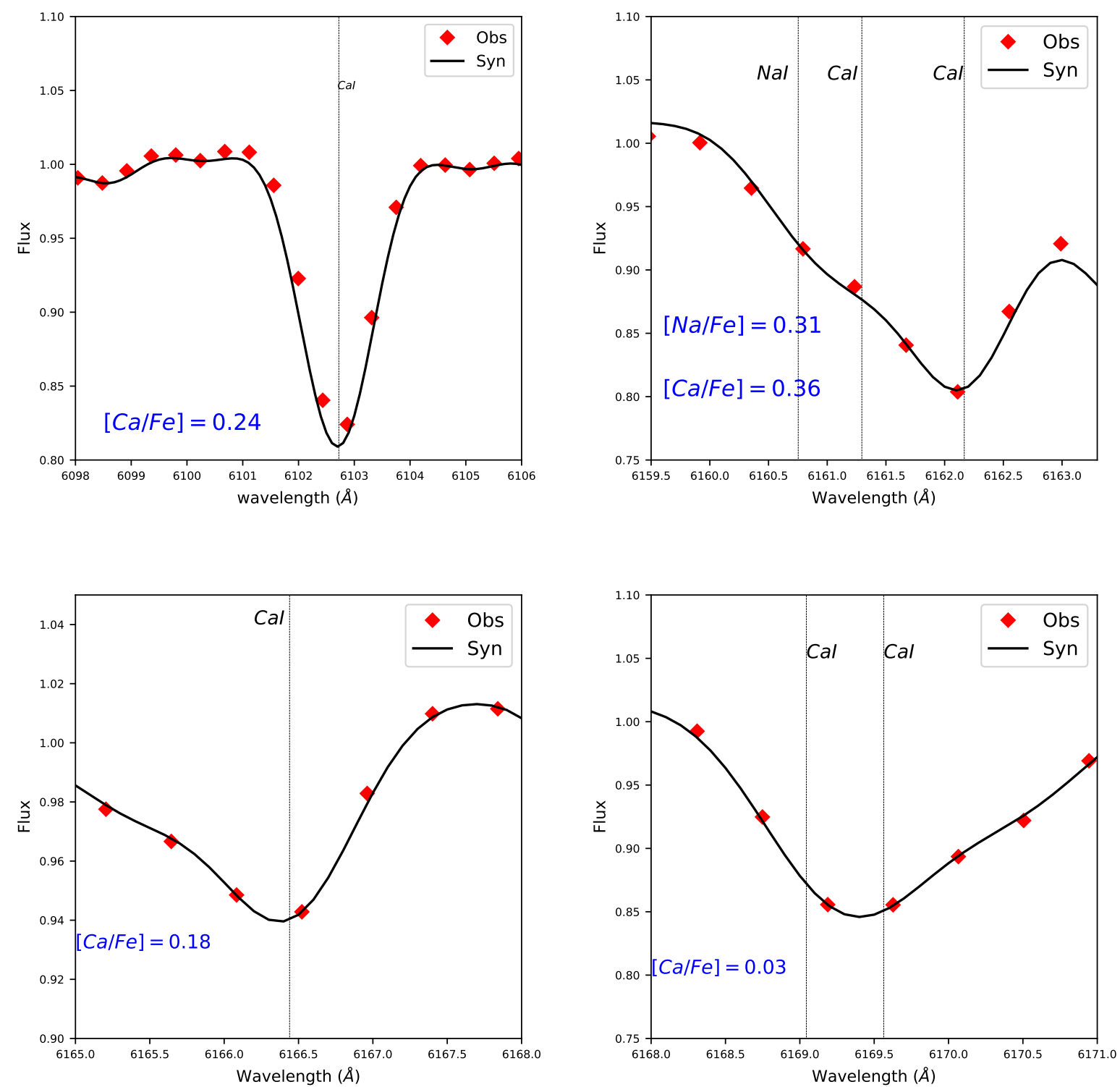

Figura 3.4: Espectros observado e sintético para as linhas de CaI em 6102.723, 6122.217, 6161.295, $6162.167,6166.44$ e 6169.044 e $6169.56 \AA$, respectivamente. 

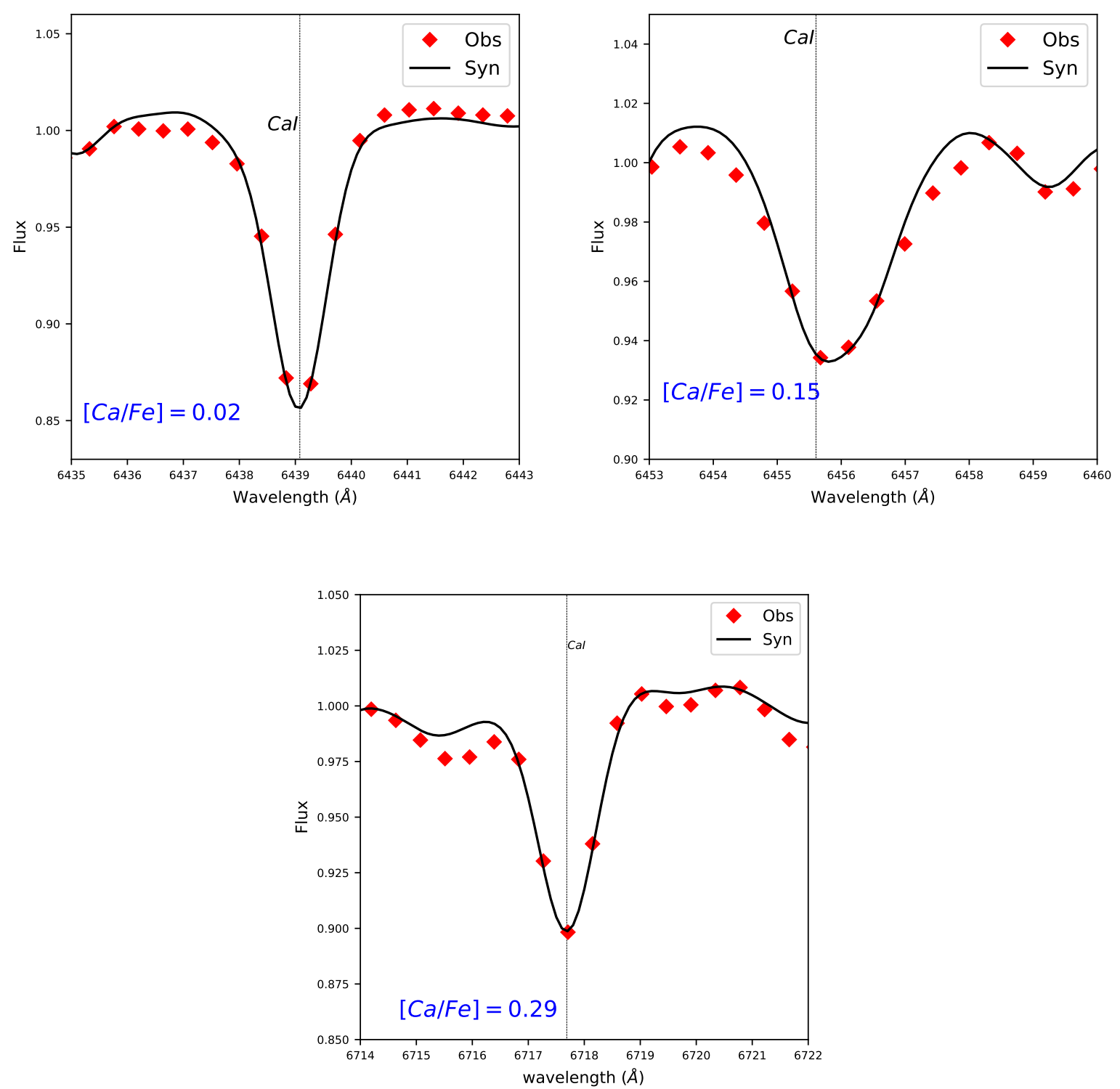

Figura 3.5: Espectros observado e sintético para as linhas de CaI em 6439.08, 6455.605, 6717.687 , respectivamente. 
na figura 3.7 e a linha localizada em $6743.127 \AA$ está apresentada na figura 3.8. A partir dessas linhas, foi obtido o valor médio para a abundância de $\mathrm{Ti}$ de $[\mathrm{Ti} / \mathrm{Fe}]=+0.30$.

\subsubsection{TiII}

As linhas de TiII localizadas em 5154.068, 5336.771, 5381.021 e $5418.751 \AA$ estão apresentadas na figura 3.9. O valor médio obtido para a abundãncia de Ti por meio dessas linhas é de $[\mathrm{Ti} / \mathrm{Fe}]=0.35$, o que mostra uma boa concordância entre os valores obtidos para a abundância do Ti utilizando linhas de diferentes estágios de ionização.

\subsubsection{Linhas do Ba}

A figura 3.10 mostra os ajustes realizados para as linhas de BaII localizadas em 5853.675, 6141.713 e $6496.9 \AA$. Efeitos relativos a estrutura hiperfina (Hyperfine Structure, HFS, do inglês) são levados em consideração, como em Barbuy et al. (2014) e Moura et al. (2019).

\subsubsection{Linha do Eu}

A figura 3.11 apresenta o ajuste feito para a linha de EuII em 6645.064 A. São levados em consideração efeitos relativos a estrutura hiperfina, como no caso das linhas de BaII.

\subsection{Linhas fortes}

Para a reprodução dos tripletos de $\mathrm{Mg}$, localizado em 5167-5183 A e para o tripleto de CaII localizado em 8498-8662 A, foram utilizados valores atualizados da literatura para as forças de oscilador, e para as constantes de amortecimento, que são importantes para o bom ajuste das asas das linhas. Os valores estão presentes na tabela 2.1 e foram extraídos de Pehlivan Rhodin et al. (2017) e Kurucz (2005a), e já discutidos e apresentados em Moura et al. (2019).

Em estrelas do tipo F e M, o principal fator responsável pelo alargamento de linhas metálicas são colisões entre esses elementos e átomos de H neutros. De acordo com Gray (2005), a expressão que correlaciona a constante de amortecimento $\gamma$ e a constante de interação $\mathrm{C}_{6}$, para cada uma das camadas atmosféricas de uma estrela é: 

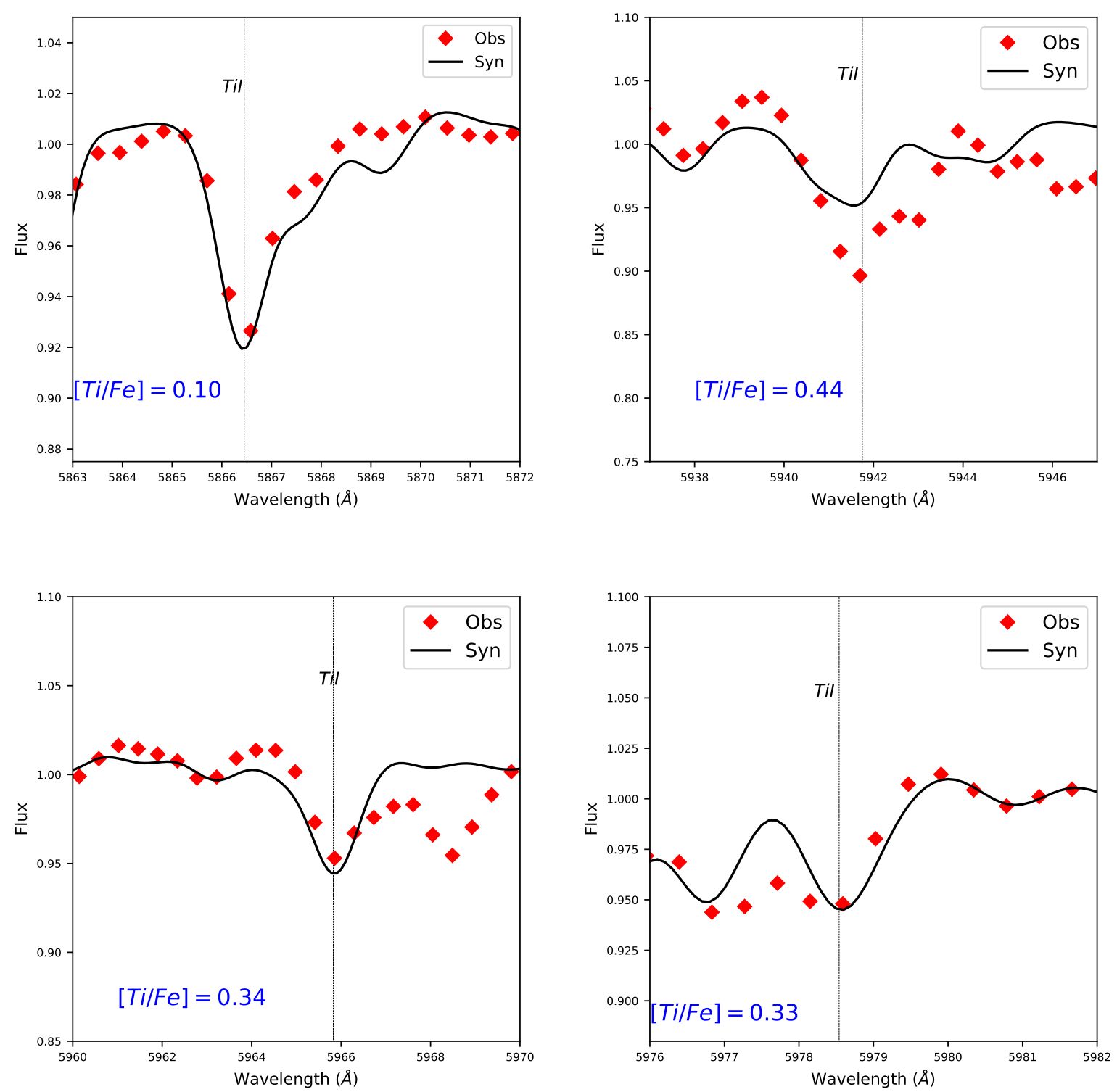

Figura 3.6: Espectros observado e sintético para as linhas de TiI em 5866.449, 5941.75, 5965.825 e 5978.539 $\AA$ A, respectivamente. 

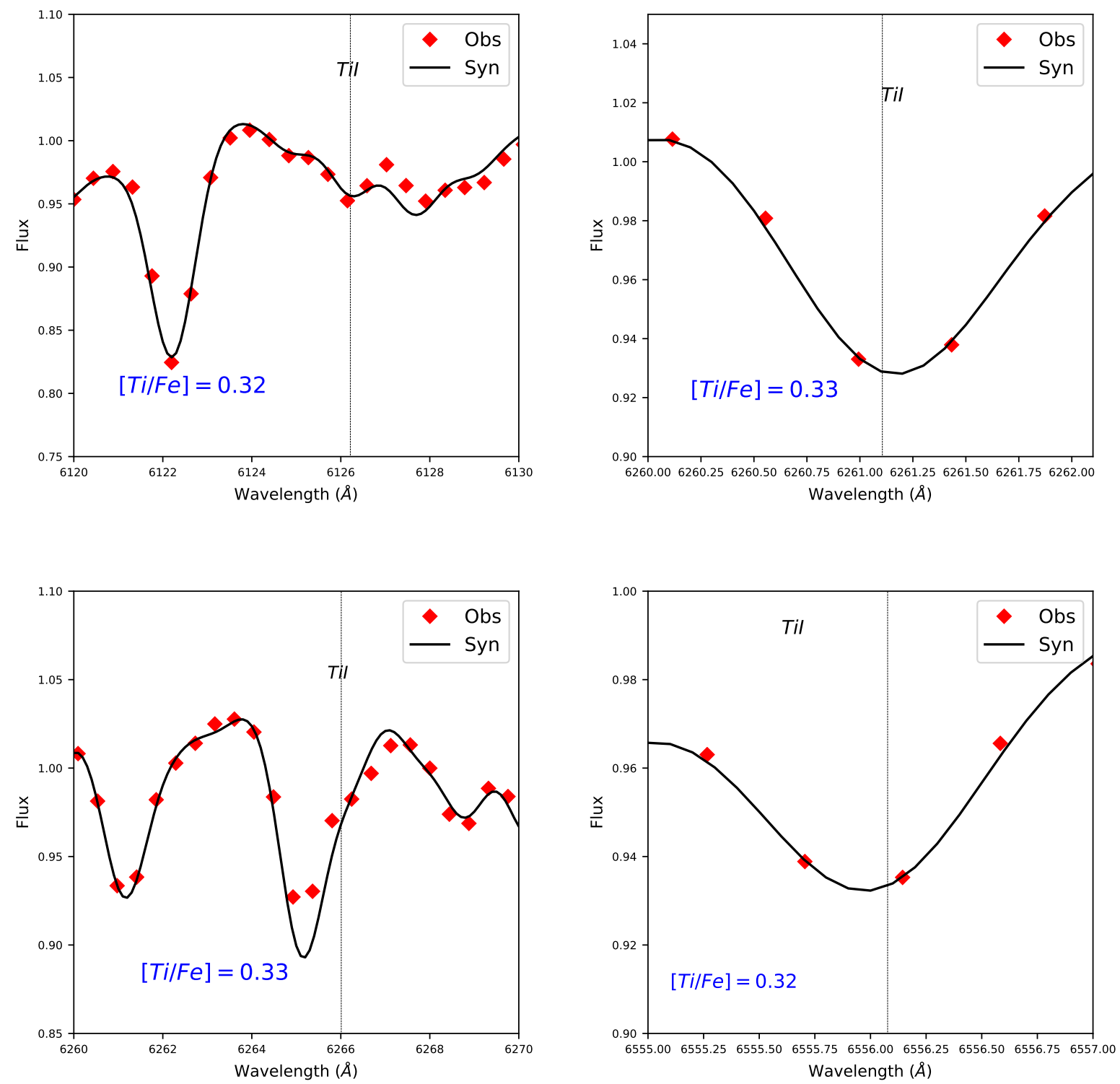

Figura 3.7: Espectros observado e sintético para as linhas de TiI em 6126.214, 6261.106, 6266.01 e 6556.077 $\AA$, respectivamente. 


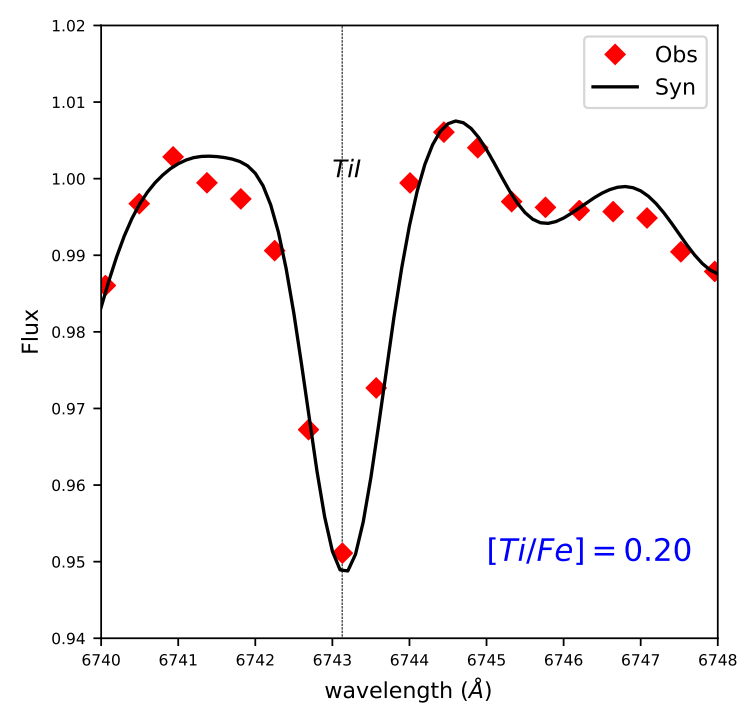

Figura 3.8: Espectros observado e sintético para a linha de TiI em $6743.127 \AA$.

$$
\gamma_{6} / N_{H}=17 v^{3 / 5} C_{6}^{2 / 5}
$$

Onde $v$ é a velocidade relativa entre os átomos que colidem e $\mathrm{N}_{\mathrm{H}}$ é a densidade de átomos de hidrogênio na estrela. O alargamento radiativo $\left(\gamma_{\mathrm{R}}=2.21 E 15 / \lambda^{2}\right.$ para a maioria das linhas), assim como o alargamento devido a elétrons $\left(\gamma_{\mathrm{e}}\right)$ também são levados em consideração para a formação do perfil de Voigt resultante (Gray (2005)). Os valores das constantes de amortecimento $\gamma_{\mathrm{R}}$ e $\gamma_{\mathrm{e}}$ para os respectivos casos foram extraídas de VALD3 (Ryabchikova et al. (2015)) e da webpage de Kurúcz $\uparrow^{\text {T }}$. Mais detalhes podem ser encontrados em Chmielewski (2000) e em Smith e Drake (1988).

Os valores adotados para a constante de amortecimento $\mathrm{C}_{6}$ foram calculados a partir do ajuste das asas das linhas, considerando os valores de força de oscilador mais recentes da literatura. Para isso, foram considerados modelos atmosféricos de MARCS Gustafsson et al. (2008) ) aplicados ao Sol (Kurucz (2005b)) e Arcturus (Hinkle et al. (2000)). Os

\footnotetext{
${ }^{1}$ http://www.cfa.harvard.edu/amp/ampdata/kurucz23/sekur.html
} 

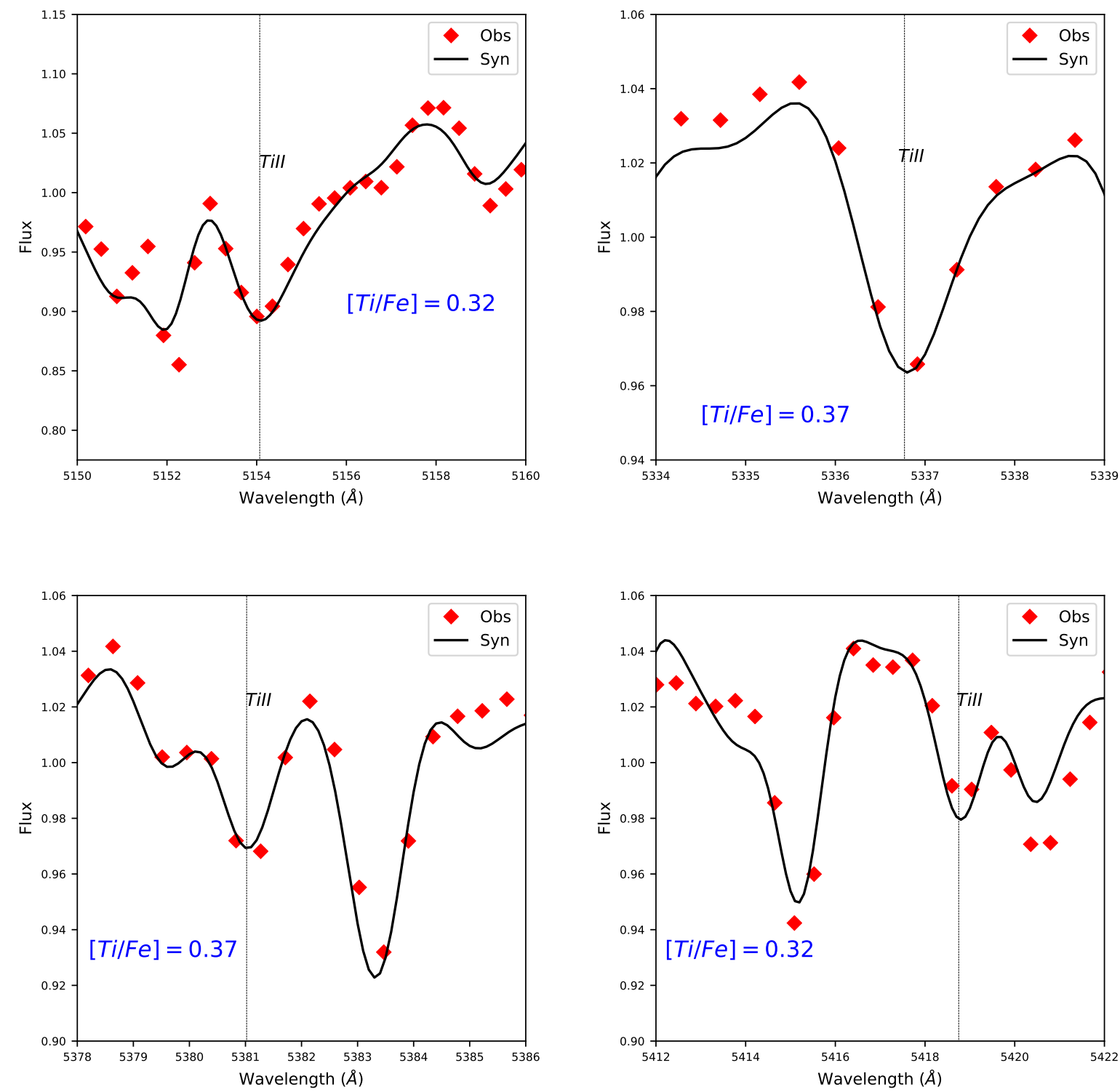

Figura 3.9: Espectros observado e sintético para as linhas de TiI em 5154.068, 5336.771, 5381.021 e $5418.751 \AA$, respectivamente. 

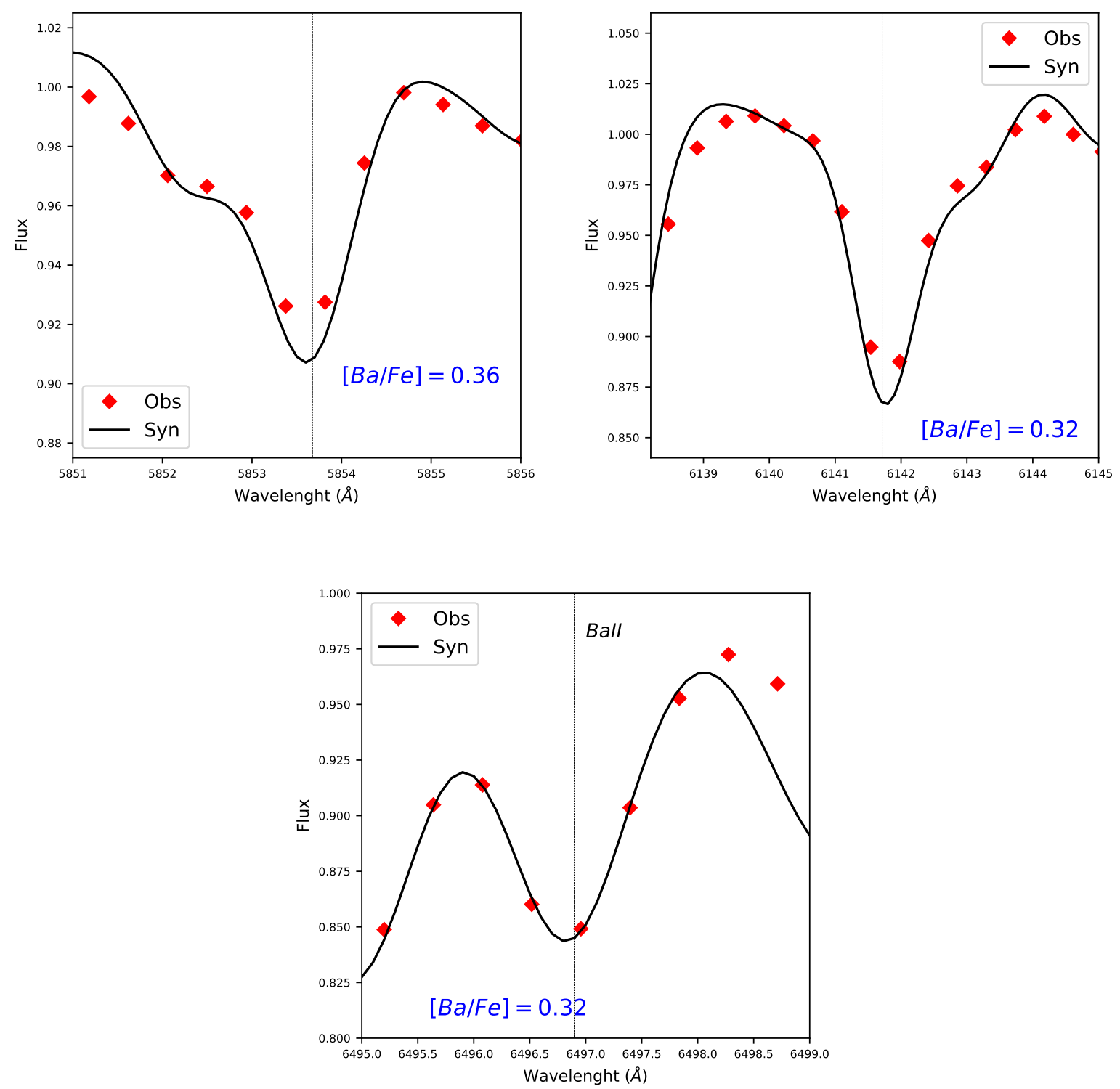

Figura 3.10: Espectros observado e sintético para as linhas de BaII em 5853.675, 6141.713 e $6496.9 \AA$, respectivamente. 


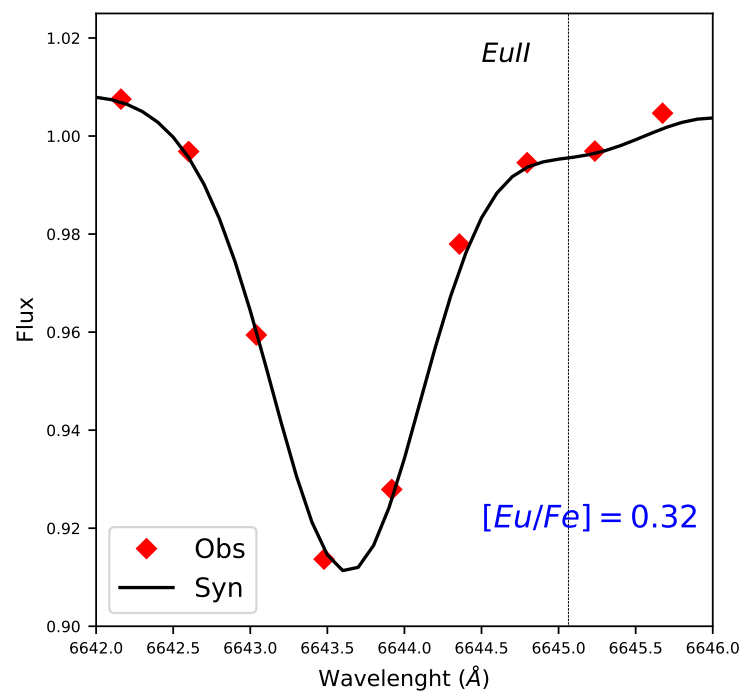

Figura 3.11: Espectros observado e sintético para a linha de EuII em $6645.064 \AA$.

parâmetros estelares adotados foram $\mathrm{T}_{\text {eff }}=5770 \mathrm{~K}, \log \mathrm{g}=4.44,[\mathrm{Fe} / \mathrm{H}]=0.0 \mathrm{e} \mathrm{v}_{\mathrm{t}}=1.0$ $\mathrm{km} . \mathrm{s}^{-1}$ para o Sol, e de $\mathrm{T}_{\text {eff }}=4275 \mathrm{~K}, \log \mathrm{g}=1.55,[\mathrm{Fe} / \mathrm{H}]=-0.54 \mathrm{e} \mathrm{v}_{\mathrm{t}}=1.65 \mathrm{~km} . \mathrm{s}^{-1}$ para Arcturus, como em Meléndez et al. (2003). Também foram adotadas abundâncias de $\mathrm{A}(\mathrm{Fe})=7.50$ para o Sol e de $[\mathrm{Mg} / \mathrm{Fe}]=+0.15$ e $[\mathrm{Ca} / \mathrm{Fe}]=0.10$ para Arcturus.

As linhas em 5167.32, 5172.68 e $5183.604 \AA$ pertencentes ao tripleto do $\mathrm{MgI}$ e as linhas em 8498.18, 8542.089 e $8662.14 \AA$ pertencentes ao tripleto do CaII (CaT) estão apresentadas nas figuras 3.12 e 3.13 . É importante ressaltar que, diferentemente das linhas anteriormente apresentadas, os tripletos não foram ajustados de modo a melhor representar o perfil da linha toda, mas sim apenas das asas, visto que o fundo das linhas não pode ser reproduzido por cálculos que consideram condições de LTE. Os fundos dessas linhas são gerados nas cromosferas estelares, em condições que estão fora do equilíbrio termodinâmico local.

\subsection{Múltiplas Populações Estelares em Aglomerados Globulares}

Múltiplas populações estelares em GCs (ou MPs, de Multiple Stellar Populations) foram identificadas por fotometria (Bedin et al. (2004)), e por espectroscopia por meio das anticorrelações encontradas entre $\mathrm{Na}-\mathrm{O}$ e $\mathrm{Mg}-\mathrm{Al}$, em conjunto com enriquecimentos de $\mathrm{N}$ e deficiências em O, que foram encontradas de forma sistemática em aglomerados globulares 

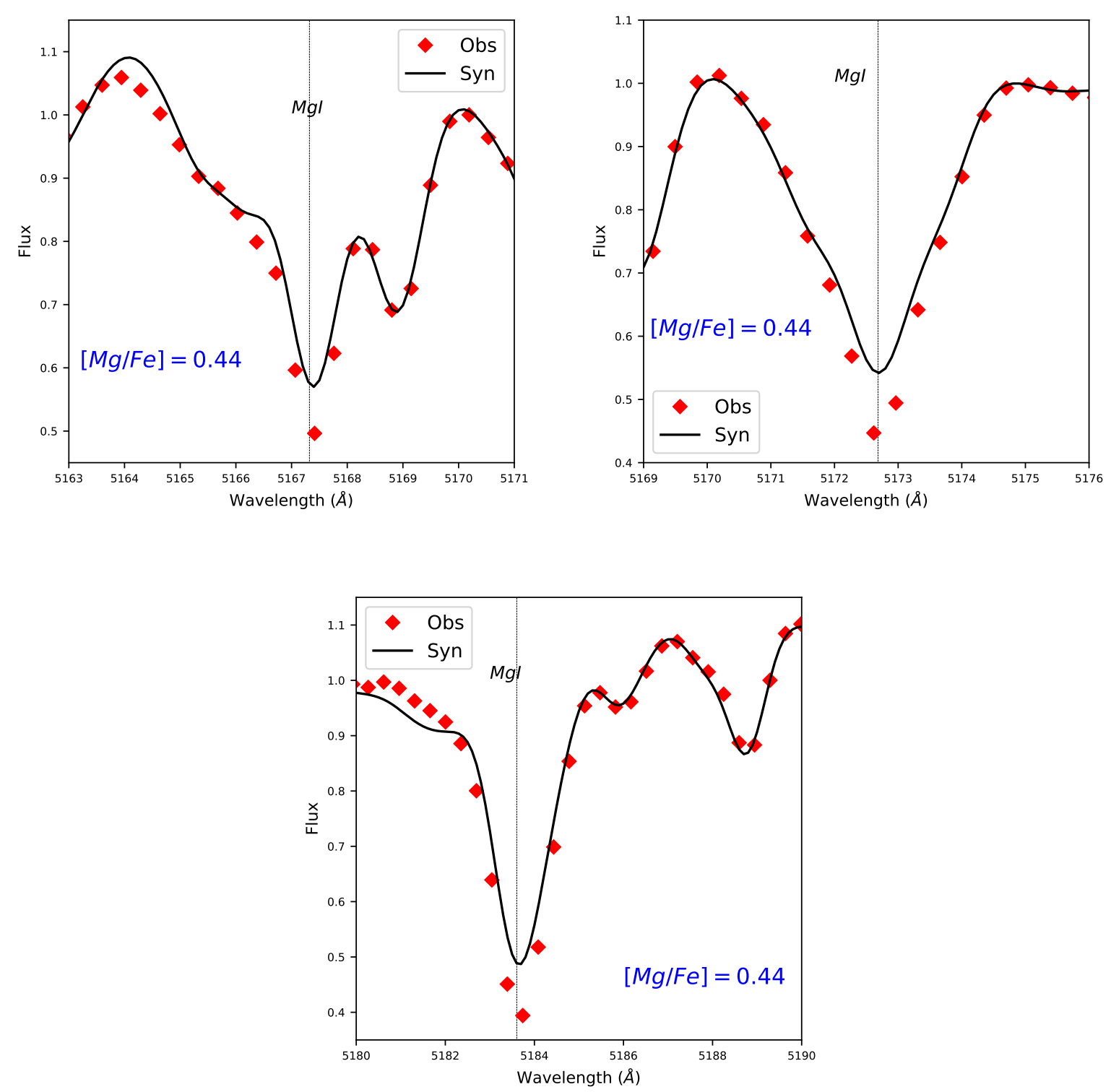

Figura 3.12: Linhas do tripleto do MgI localizadas em 5167.32, 5172.68 e 5183.604 A. respectivamente. 

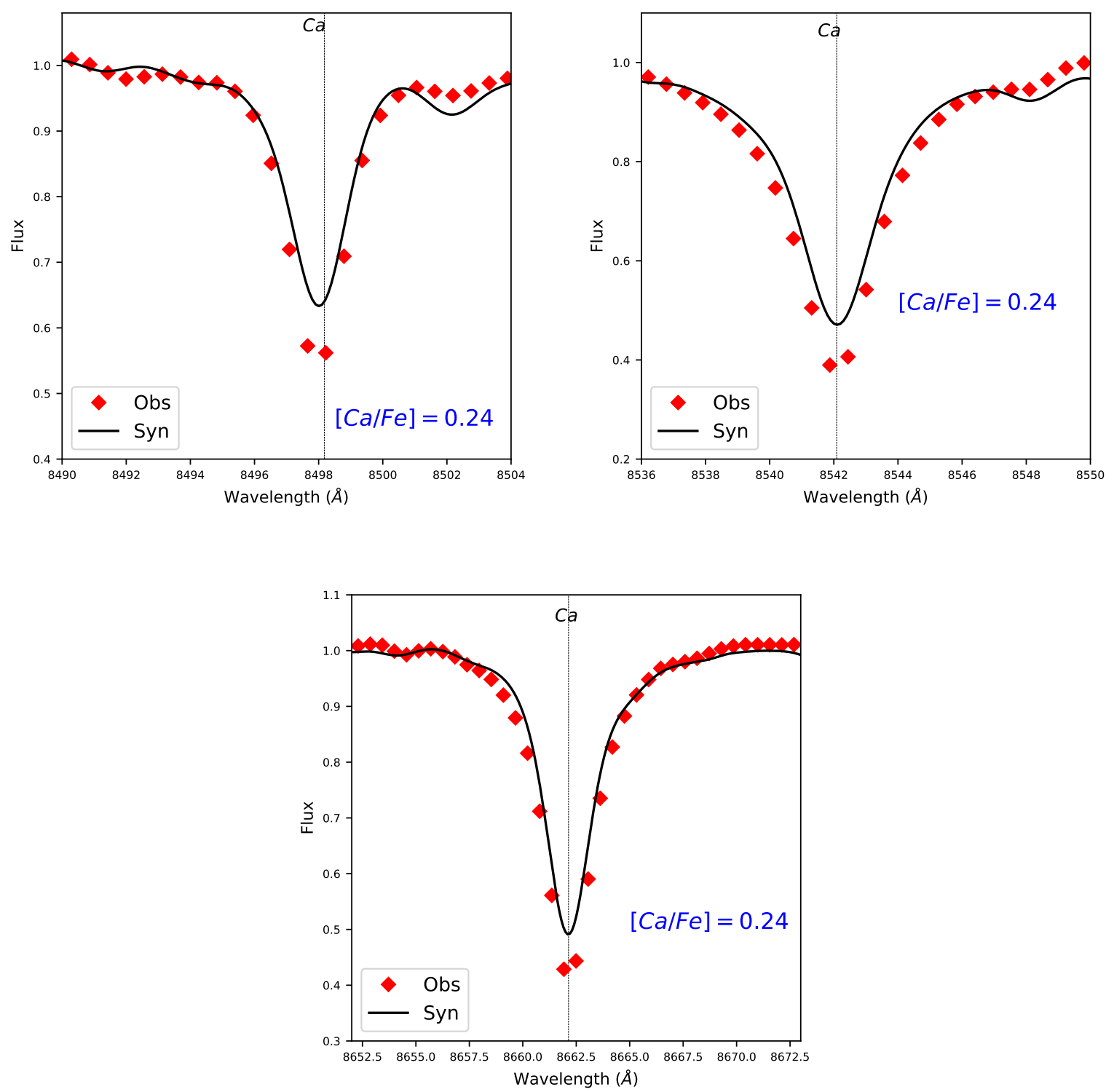

Figura 3.13: Linhas do tripleto do CaII localizadas em 8498.18, 8542.089 e 8662.14 A. respectivamente. 
(vide reviews de Gratton et al. (2004) e Gratton et al. (2012)). Piotto et al. (2015)) e Milone et al. (2017) demostraram que essencialmente todos os aglomerados globulares apresentam uma primeira (1G) e uma segunda geração $(2 \mathrm{G})$ de estrelas. Para o 47 Tuc, a razão entre o número de estrelas da primeira geração e o número total de estrelas é de $\mathrm{N}_{1} / \mathrm{N}_{T O T}=0.175$.

Na é um grande indicador de MPs, pois as segundas gerações estelares em GCs apresentam abundâncias altas de $\mathrm{Na}$, como demonstrado em in Carretta (2019). Em espectros integrados, a presença de múltiplas populações de estrelas seria detectada por meio de um aumento na abundância média de $\mathrm{Na}$ observada. Para checar essa possibilidade, foram calculados espectros sintéticos para $[\mathrm{Na} / \mathrm{Fe}]=0.0,0.3$ e para a abundância que melhor se ajusta ao espectro observado para seis linhas selecionadas de Na. Os resultados estão indicados na Figuras 3.14 e 3.15. Em vista da variação do resultado conforme a linha, não foi possível concluir através dessa análise a presença de múltiplas populações no 47 Tuc.

\subsection{Abundâncias de He}

Para a confecção de espectros integrados com o SynSSP, são utilizadas isócronas de Dartmouth com abundâncias de He primordiais, ou seja, Y $=0.247$. São esperados aumentos nas abundâncias de Hélio encontradas nas estrelas pertencentes a 2G (por exemplo, Lagioia et al. (2018)), porém, como a variação de He para a 2G é pequena em relação ao valor primordial (vide Milone et al. (2019)), e como demonstrado na Figura 3.16 extraída de Souza et al. (2020), pequenas variações nas abundâncias de He não alteram de forma muito significativa as isócronas, ou seja, se considerado enriquecimento de He correspondentes a $2 \mathrm{G}$ de estrelas, as isócronas seriam muito próximas às utilizadas originalmente.

\subsection{Efeitos da Idade}

É bem conhecido o fato de que a idade de uma certa população estelar pode ser inferida por meio de um diagrama de Hertzsprung - Russell, com base no ponto onde acaba sua sequência principal e começa seu ramo das gigantes (esse ponto é conhecido como ponto de turnoff). A idade é um dos ingredientes cruciais para a construção de uma isócrona teórica, porém, em espectros integrados de aglomerados globulares, o fluxo é geralmente dominado pela luz oriunda de estrelas gigantes. Na Figura 3.17 estão sobrepostos espectros 

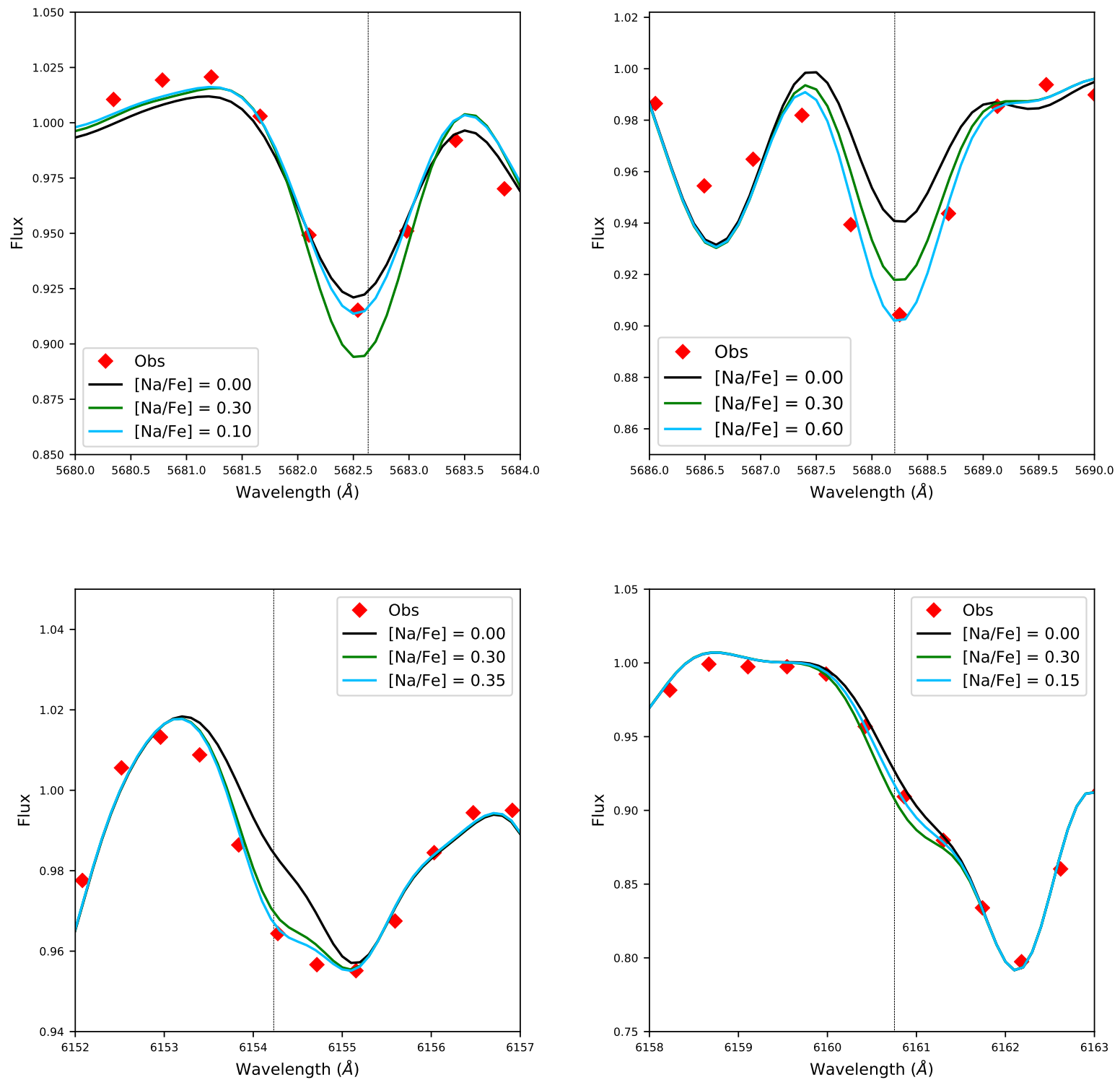

Figura 3.14: Ajuste entre espectro observado e espectros sintéticos com $[\mathrm{Na} / \mathrm{Fe}]=0.0,0.3$ e melhor valor de ajuste para as linhas de NaI localizadas em 5682.633, 5688.205, 6154.230 e $6160.753 \AA$, respectivamente. 

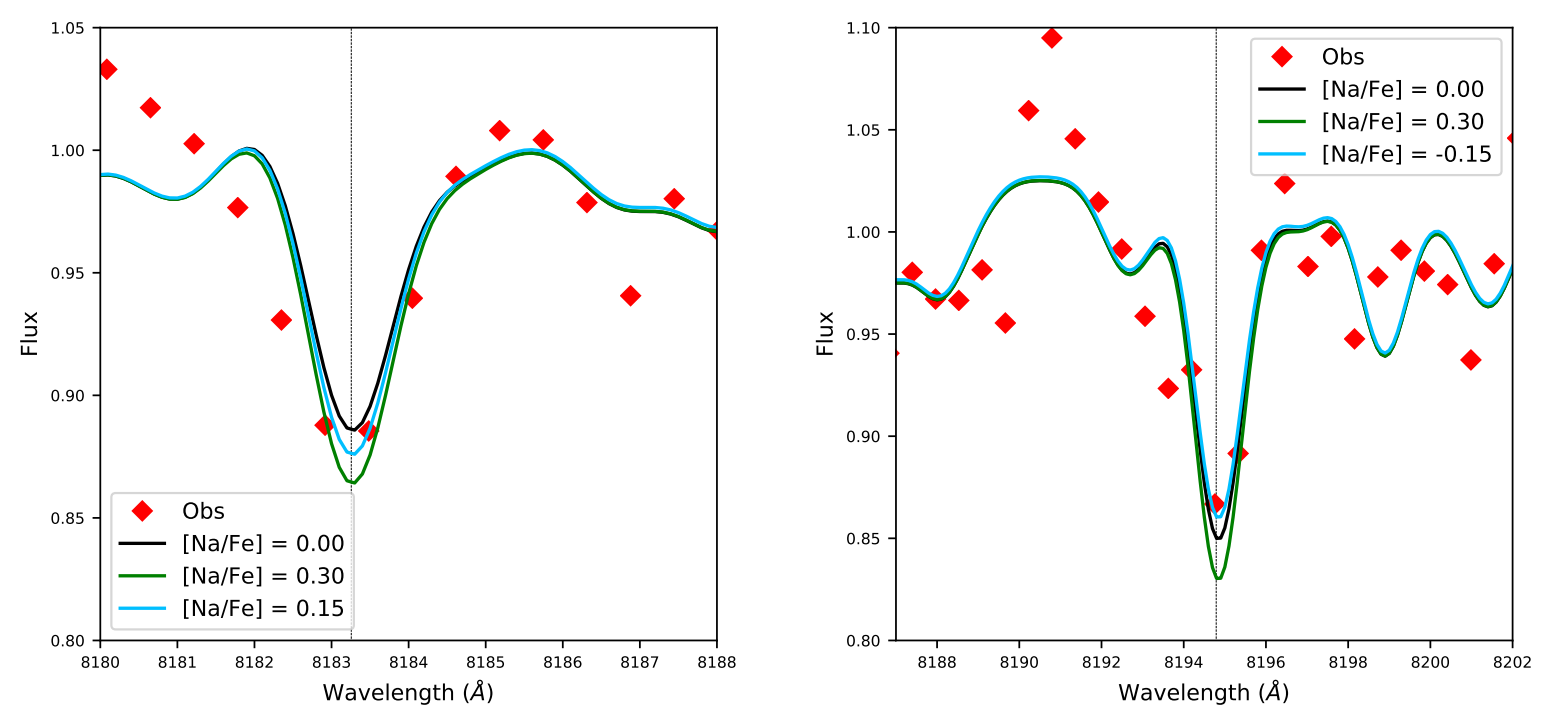

Figura 3.15: Ajuste entre espectro observado e espectros sintéticos com $[\mathrm{Na} / \mathrm{Fe}]=0.0,0.3$ e melhor valor de ajuste para as linhas de NaI localizadas em 8183.256 e $8194.790 \AA$, respectivamente.
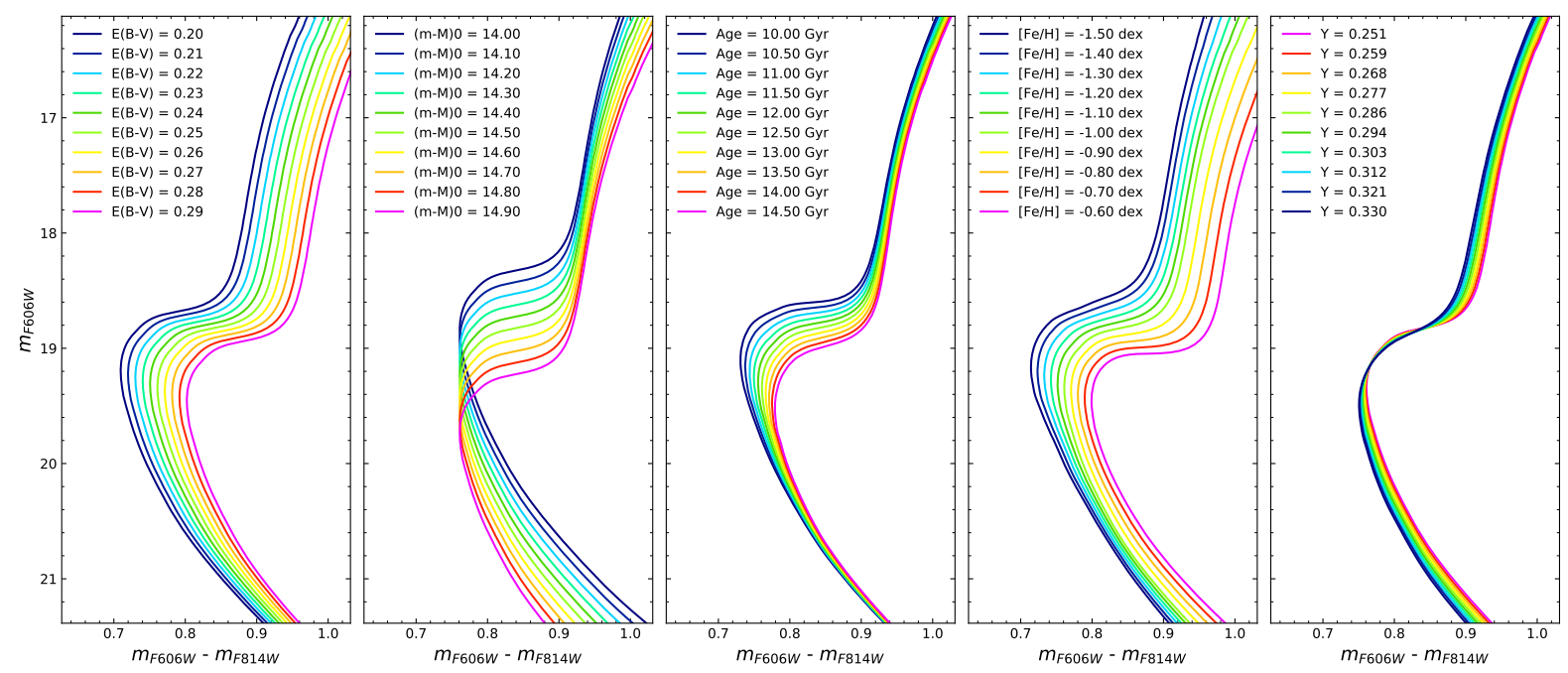

Figura 3.16: Explicação gráfica de como mudanças em E(B - V), módulo de distância, idade, metalicidade e abundância de He, respectivamente, afetam a morfologia e posição de isócronas. Extraído de Souza et al. (2020). 
integrados sintéticos no intervalo 5000 - $5500 \AA$, com idades de 11 e 13 Gyrs e abundâncias elementais idênticas, além da diferença entre seus fluxos, chamados de resíduos. A Figura 3.17 apresentam uma sobreposição entre espectros integrados com idades de 11 e 13 Gyr, e também do espectro observado, no intervalo 4500 - $9000 \AA$.

\subsection{Discussão}

As abundâncias médias para as linhas ajustadas estão apresentadas na tabela 3.1 . Os valores encontrados por meio da técnica utilizada nesse trabalho são bastante compatíveis com os valores encontrados em trabalhos anteriores, mesmo considerando os trabalhos que calcularam abundâncias por meio de estrelas individuais.

Algumas das linhas utilizadas nesse trabalho também foram utilizadas em Colucci et al. (2017), e estão listadas na tabela 3.2. Nota-se que os resultados foram parecidos para as linhas de $\mathrm{Mg}, \mathrm{Al}$ e $\mathrm{Eu}$, mas razoavelmente diferentes para as outras linhas em comum. Porém, ao analisarmos as médias encontradas em ambos os trabalhos (vide figura 3.18), os resultados são semelhantes, com exceção das abundâncias encontradas para o Mg.

Na figura 3.18 estão listados os valores das abundâncias médias apresentadas nos trabalhos listados na tabela 1.2 . Os resultados obtidos na análise apresentada são bastante consistentes com os valores previamente obtidos para as abundâncias dos elementos investigados, sendo consistentes mesmo com os trabalhos que calcularam abundâncias elementais a partir de espectros de estrelas individuais, com exceção da abundância do Na, que é o elemento que possui a maior discrepância entre os valores obtidos em diferentes trabalhos.

A partir das linhas do $\mathrm{Na}$ analisadas, as abundâncias obtidas para o $\mathrm{Na}$ foram de +0.10 para a linha em $5682.63 \AA,+0.60$ para a linha em $5688.20 \AA,+0.35$ para a linha em $6154.23 \AA,+0.15$ para a linha em $6160.75 \AA,+0.15$ para a linha em $8183.25 \AA$ e de -0.15 para a linha em 8183.25 Å. Considerando que em estrelas de segunda geração, há um

Tabela 3.1 - Metalicidade adotada e abundâncias médias calculadas por ajuste de linhas, para os elementos $\mathrm{Mg}, \mathrm{Al}, \mathrm{Si}, \mathrm{Ca}, \mathrm{Ti}, \mathrm{Ba}$ e Eu.

\begin{tabular}{lllllllll}
{$[\mathrm{Fe} / \mathrm{H}]$} & {$[\mathrm{Mg} / \mathrm{Fe}]$} & {$[\mathrm{Al} / \mathrm{Fe}]$} & {$[\mathrm{Si} / \mathrm{Fe}]$} & {$[\mathrm{Ca} / \mathrm{Fe}]$} & {$[\mathrm{TiI} / \mathrm{Fe}]$} & {$[\mathrm{TiII} / \mathrm{Fe}]$} & {$[\mathrm{Ba} / \mathrm{Fe}]$} & {$[\mathrm{Eu} / \mathrm{Fe}]$} \\
\hline$-0.79 \pm 0.07$ & $0.45 \pm 0.01$ & $0.24 \pm 0.01$ & $0.30 \pm 0.07$ & $0.20 \pm 0.04$ & $0.30 \pm 0.03$ & $0.35 \pm 0.02$ & $0.33 \pm 0.02$ & $0.32 \pm 0.01$ \\
\hline
\end{tabular}



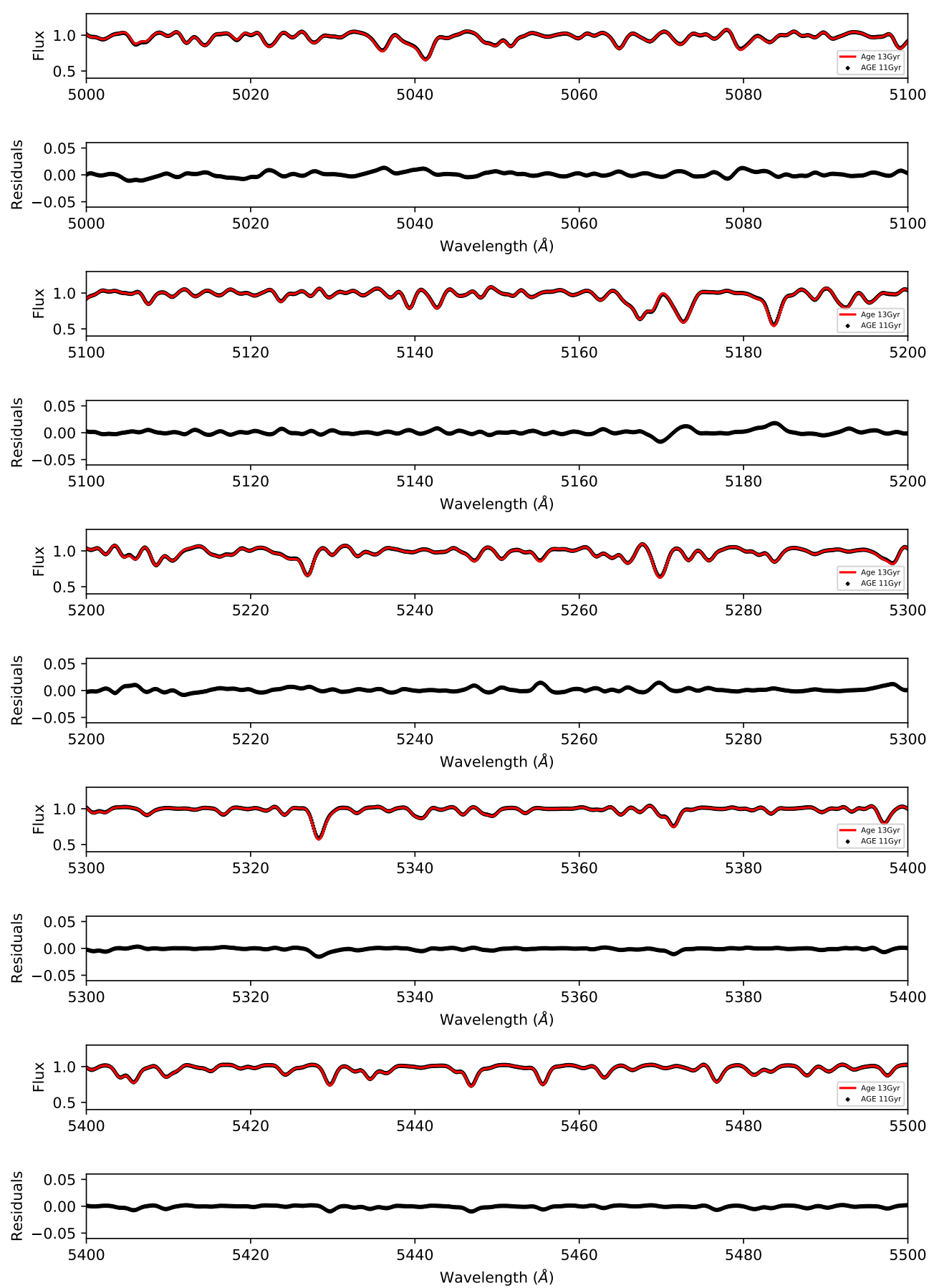

Figura 3.17: Espectros integrados para o 47 Tuc com abundâncias elementais idênticas e idades de 11 e 13 Gyrs, além de seus respectivos resíduos, no intervalo 5000 - $5500 \AA$. 
enriquecimento de Na que varia entre 0.2 e 0.7 (como mostrado em Campbell et al. (2013)), e que a razão entre as estrelas da primeira geração e o número total de estrelas no 47 Tuc é de 0.175, como mostrado em Milone et al. (2017), os valores de abundância obtidos por meio dos ajustes realizados são inconclusivos quanto a determinar a incidência de múltiplas populações estelares no 47 Tuc. As bandas espectrais onde as linhas escolhidas estão localizadas possuem uma razão sinal-ruído média acima de 600, e uma resolução $\mathrm{R}=6,800$ ou $\Delta \lambda \sim 1 \AA^{-1}$, então espectros integrados observados que tenham uma maior resolução espectral provavelmente permitiriam a determinação de MPs através de medidas de $[\mathrm{Na} / \mathrm{Fe}]$, a partir de linhas individuais.

\subsubsection{Incertezas associadas}

Uma estimativa precisa do erro associado a um método é algo bastante complexo de se determinar. No presente caso, existem erros oriundos de cada uma das escolhas de parâmetros utilizados para a construção do espectro sintético, e não é simples precisar o peso real de cada um desses ingredientes no resultado final. Nesse trabalho iremos adotar uma forma simplificada do erro, que irá levar em consideração apenas a dispersão dos resultados obtidos para as abundâncias para cada elemento.

Como pode ser observado na figura 3.17, e nas figuras B.1 - B.9 do Apêndice B, a diferença entre os espectros integrados gerados para as estimativas de idades máxima e mínima do 47 Tuc previamente apresentadas é pequena, variando entre 2 e $5 \%$ do fluxo espectral. Como o espectro é dominado pelo fluxo de estrelas gigantes, os efeitos de mudança no espectro integrado devido à presença de estrelas na sequência principal é menos importante.

O erro associado com a dispersão é dado pelo desvio padrão da média, como em Colucci et al. (2017), é dado por

$$
\sigma_{X, N}=\sigma / \sqrt{\left(N_{X}-1\right)}
$$


Tabela 3.2 - Comparação entre os valores obtidos neste trabalho e em Colucci et al. (2017). É importante salientar que a metalicidade adotada neste trabalho foi de $[\mathrm{Fe} / \mathrm{H}]=-0.79$, enquanto em Colucci et al. (2017) o valor é de $[\mathrm{Fe} / \mathrm{H}]=-0.65$

\begin{tabular}{lccccc}
\hline \hline Espécie & $\lambda(\AA)$ & $\mathrm{A}(\mathrm{X})_{\odot \text { Colucci }}$ & $\mathrm{A}(\mathrm{X})_{\text {Colucci }}$ & {$[\mathrm{X} / \mathrm{Fe}]_{\text {Colucci }}$} & {$[\mathrm{X} / \mathrm{Fe}]_{\text {essetrabalho }}$} \\
\hline $\mathrm{NaI}$ & 5682.633 & 6.44 & 5.86 & +0.07 & +0.10 \\
$\mathrm{NaI}$ & 5688.200 & 6.34 & 5.96 & +0.27 & +0.60 \\
$\mathrm{NaI}$ & 6154.230 & 6.24 & 5.86 & +0.27 & +0.35 \\
$\mathrm{MgI}$ & 5528.405 & 7.52 & 7.22 & +0.35 & +0.38 \\
$\mathrm{AlI}$ & 6698.673 & 6.46 & 6.07 & +0.26 & +0.24 \\
$\mathrm{SiI}$ & 5948.55 & 7.61 & 7.23 & +0.27 & +0.42 \\
$\mathrm{SiI}$ & 7415.96 & 7.56 & 7.62 & +0.71 & +0.32 \\
$\mathrm{SiI}$ & 7423.51 & 7.60 & 7.43 & +0.48 & +0.12 \\
$\mathrm{CaI}$ & 6102.723 & 6.45 & 5.83 & +0.03 & +0.24 \\
$\mathrm{CaI}$ & 6166.440 & 6.36 & 5.83 & +0.12 & +0.18 \\
$\mathrm{CaI}$ & 6439.080 & 6.02 & 5.63 & +0.26 & +0.02 \\
$\mathrm{CaI}$ & 6455.605 & 6.29 & 5.73 & +0.09 & +0.15 \\
TiI & 5866.449 & 5.04 & 4.57 & +0.18 & +0.10 \\
TiI & 6556.077 & 5.41 & 4.77 & +0.01 & +0.32 \\
TiII & 5336.077 & 5.15 & 4.77 & +0.27 & +0.37 \\
TiII & 5381.021 & 5.21 & 4.67 & +0.11 & +0.37 \\
EuII & 6645.064 & 0.42 & 0.14 & +0.37 & +0.32 \\
\hline
\end{tabular}

Os resultados das abundâncias obtidas para cada uma das linhas analisadas, assim como seu comprimento de onda e seus dados básicos estão listados na tabela 3.3 . Os resultados finais para as abundâncias médias, assim como o seu erro associado estão listados na tabela 3.1 .

Uma forma de se obter uma incerteza mais precisa, que leva em consideração os efeitos dos erros associados as parâmetros usados para a construção da SSP seria reproduzir as linhas da lista analisada para ambas as idades máxima e mínima do 47 Tuc, e comparar o efeito da disparidade de fluxos gerados nas abundâncias. A partir desses resultados, poderíamos observar qual é a influência da diferença de fluxos nas abundâncias, e a partir disso integrar esse efeito no erro total, porém, esse teste não foi realizado para esse trabalho. 


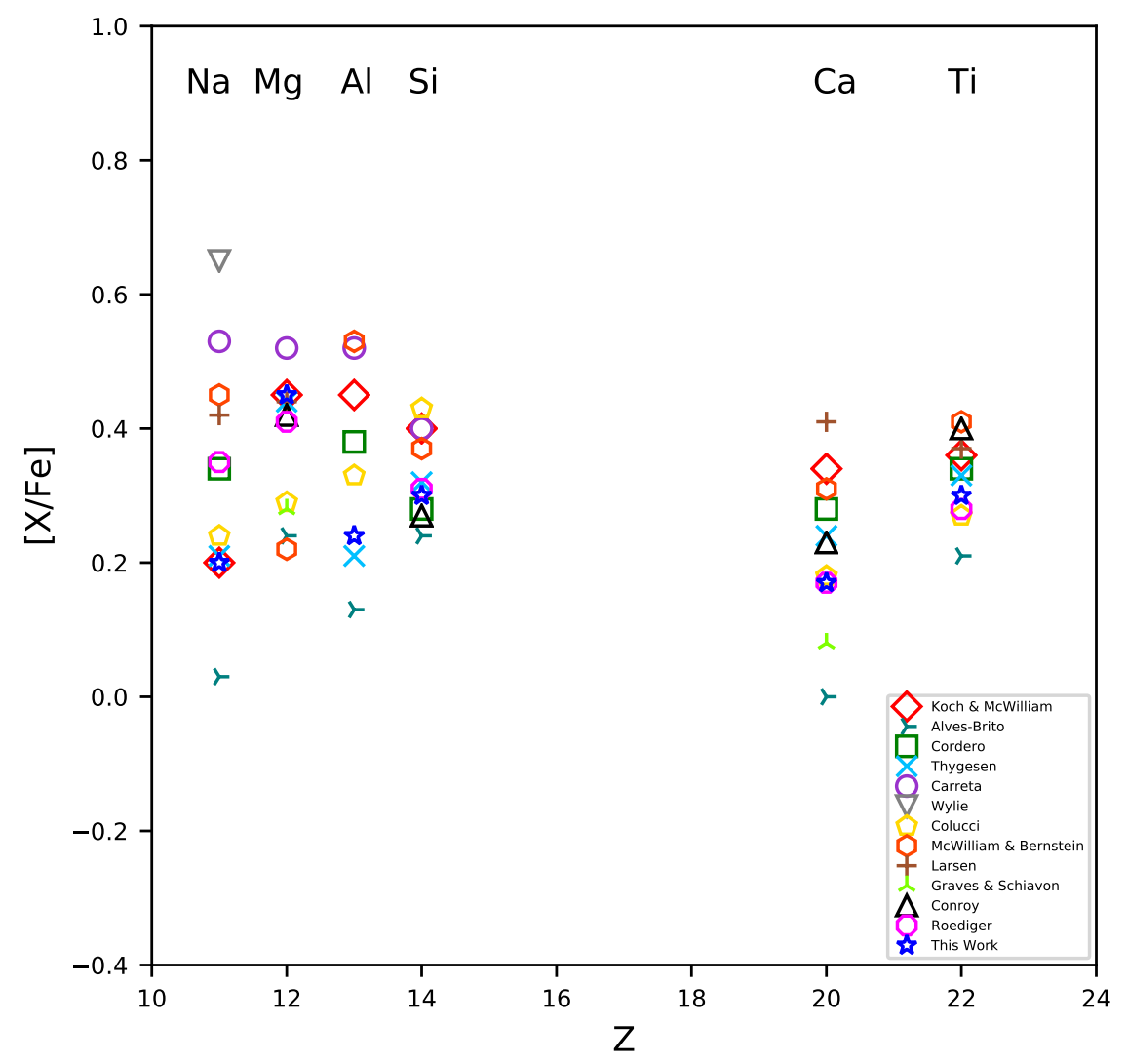

Figura 3.18: $[\mathrm{X} / \mathrm{Fe}]$ versus $\mathrm{Z}$ para as abundâncias calculadas nesse trabalho e para os valores previamente calculados em outros trabalhos. A estrela azul indica os resultados obtidos nesse trabalho. 
Tabela 3.3 - Lista de linhas analisadas no espectro, e abundâncias resultantes.

\begin{tabular}{|lcccc|}
\hline Espécie & $\lambda(\AA)$ & $\chi_{\text {ex }}(\mathrm{eV})$ & log gf & 47 Tuc \\
\hline NaI & 5682.633 & 2.10 & -0.71 & 0.10 \\
NaI & 5688.205 & 2.11 & -0.45 & 0.60 \\
NaI & 6154.230 & 2.10 & -1.56 & 0.35 \\
NaI & 6160.753 & 2.10 & -1.26 & 0.15 \\
NaI & 8183.256 & 2.10 & -0.47 & 0.15 \\
NaI & 8194.790 & 2.10 & +0.24 & -0.15 \\
MgI & 5528.405 & 4.34 & -0.547 & 0.46 \\
MgI & 8806.756 & 4.34 & -0.144 & 0.44 \\
AlI & 6696.185 & 4.02 & -1.58 & 0.24 \\
AlI & 6696.204 & 4.02 & -1.58 & 0.24 \\
AlI & 6696.788 & 4.02 & -1.42 & 0.24 \\
AlI & 6698.673 & 3.14 & -1.65 & 0.24 \\
SiI & 5948.55 & 5.08 & -1.23 & 0.42 \\
SiI & 7405.79 & 5.61 & -0.66 & 0.32 \\
SiI & 7415.96 & 5.61 & -0.73 & 0.32 \\
SiI & 7423.51 & 5.62 & -0.58 & 0.12 \\
CaI & 6102.723 & 1.88 & -0.79 & 0.24 \\
CaI & 6122.217 & 1.89 & -0.20 & 0.36 \\
CaI & 6161.295 & 2.51 & -1.02 & 0.36 \\
CaI & 6162.167 & 1.89 & -0.09 & 0.36 \\
CaI & 6166.440 & 2.52 & -0.90 & 0.18 \\
CaI & 6169.044 & 2.52 & -0.54 & 0.03 \\
CaI & 6169.564 & 2.52 & -0.27 & 0.03 \\
CaI & 6439.080 & 2.52 & +0.3 & 0.02 \\
CaI & 6455.605 & 2.52 & -1.35 & 0.15 \\
CaI & 6717.687 & 2.71 & -0.61 & 0.29 \\
TiI & 5866.449 & 1.07 & -0.84 & 0.10 \\
TiI & 5922.108 & 1.05 & -1.46 & 0.33 \\
TiI & 5941.750 & 1.05 & -1.53 & 0.44 \\
TiI & 5965.825 & 1.88 & -0.42 & 0.34 \\
TiI & 5978.539 & 1.87 & -0.53 & 0.33 \\
TiI & 6126.214 & 1.07 & -1.43 & 0.32 \\
TiI & 6261.106 & 1.43 & -0.48 & 0.33 \\
TiI & 6266.010 & 1.75 & -2.98 & 0.33 \\
TiI & 6556.077 & 1.46 & -1.07 & 0.32 \\
TiI & 6743.127 & 0.90 & -1.73 & 0.20 \\
TiII & 5154.068 & 1.57 & -1.75 & 0.32 \\
TiII & 5336.771 & 1.58 & -1.70 & 0.37 \\
TiII & 5381.021 & 1.57 & -2.08 & 0.37 \\
TiII & 5418.751 & 1.58 & -2.13 & 0.32 \\
BaII & 5853.675 & 0.604 & -1.1 & 0.36 \\
BaII & 6141.713 & 0.704 & -0.08 & 0.32 \\
BaII & 6496.90 & 0.60 & -0.32 & 0.32 \\
EuII & 6645.064 & 1.38 & +0.12 & 0.32 \\
\hline & & & & \\
\hline
\end{tabular}


Capítulo 4

\section{Conclusões}

Através do pacote SynSSP, construído pelo nosso grupo de pesquisa, foi possível a construção de espectros integrados de aglomerados globulares velhos, a partir das suas idades, metalicidades, $[\alpha / \mathrm{Fe}]$ e sua respectiva isócrona. Os resultados podem ser encontrados em Moura et al. (2019), que usaram essa técnica para calcular o espectro integrado de um conjunto de 6 aglomerados, e nesse trabalho, que buscou fazer uma análise focada no 47 Tucanae, que é um objeto que dispõe de vasta bibliografia prévia, e portanto é um bom objeto para a realização de testes de novas técnicas de análise.

A partir da presente análise, foi possível concluir que é possível calcular abundâncias elementais em espectros integrados de GCs a partir do ajuste de linhas fracas, de forma semelhante a estudos de espectros em alta resolução, ou seja, ajustando o contínuo na vizinhança da linha de interesse, e ajustando a abundância elemental adequada que se adequa ao perfil da linha observada. Utilizando essa técnica, foi possível calcular abundâncias de $\mathrm{Na}, \mathrm{Mg}, \mathrm{Al}, \mathrm{Si}, \mathrm{Ca}, \mathrm{Ti}, \mathrm{Ba}$, e Eu, que apresentam boa coerência com os resultados de trabalhos previamente publicados com outras técnicas, inclusive àquelas que obtiveram abundâncias por meio de espectros de estrelas individuais. O valor de abundância encontrado que está em maior concordância com resultados prévios é o do Ti, onde ambas as análises de linhas de TiI e TiII apresentaram boa concordância com os trabalhos anteriores.

O resultado para abundância das linhas selecionadas de $\mathrm{Na}$ apresentou a maior discordância com os valores obtidos por trabalhos anteriores, porém, é importante notar que esse é o elemento que possui a maior discordância de resultados na literatura, como pôde ser visto na figura 3.18 . Isso provavelmente é devido ao efeito de múltiplas populações em aglomerados globulares. Por outro lado, como as diferentes linhas de Na indicam abundâncias diferentes, não foi possível a constatação da existência de múltiplas populações estelares 
por meio da análise da abundância do Na a partir das linhas selecionadas. Esse efeito deveria ser observado, uma vez que existe vasta bibliografia reportando a presença de MPs no 47 Tuc. O fato desse efeito não ter sido observado através da técnica utilizada pode indicar que talvez a resolução do espectro observado não seja suficiente para essa determinação.

Através dos resultados apresentados em Souza et al. (2020) (vide Figura 3.16), e dos resultados apresentados em Milone et al. (2017), foi possível constatar que, para o caso de GCs velhos, o enriquecimento de He presente em estrelas de segunda geração não é significativo para as isócronas utilizadas na montagem de SSPs. Esse efeito é mais presente em estrelas mais jovens e menos evoluídas, e pode ser desprezado para análises de objetos que são dominados por estrelas mais velhas e mais evoluídas.

Os efeitos devidos à idade das isócronas utilizadas não foram significativos. A partir das Figuras 3.17 e B.1 - B.9 do apêndice B, pode-se notar que os espectros obtidos para idades de 11 e 13 Gyrs apresentaram diferenças muito pouco expressivas entre si. Esse efeito também seria muito mais importante na construção de espectros de populações mais jovens e mais azuis.

Utilizando novos valores para as forças de oscilador e constantes de amortecimento, foi possível um bom ajuste das asas dos tripletos de MgI em 5167 - 5183 A e CaII em 8498 - 8662 Å. Essas linhas não foram utilizadas para cálculos de abundâncias, porém, esse bom ajuste das asas pode contribuir para o uso dessas linhas com essa finalidade, como demonstrado em Usher et al. (2019), no caso do CaII.

Os resultados apresentados são promissores, pois abrem possibilidades da utilização desse pacote para modelamento de espectros de GCs extragalácticos, o que é um passo importante na direção da análise de galáxias em si, que poderiam ser modeladas a partir da combinação de várias SSPs, uma vez que são objetos mais complexos, e que contêm diferentes grupos de estrelas, que foram formados por meio de mecanismos distintos. Uma futura utilização dessa técnica visa empregar combinações de espectros sintéticos de GCs, gerados com o SynSSP e previamente testados, para a construção de espectros integrados de galáxias externas. 


\section{Referências Bibliográficas}

Alvarez R., Plez B., Near-infrared narrow-band photometry of M-giant and Mira stars: models meet observations, A\&A, 1998, vol. 330, p. 1109

Alves-Brito A., Barbuy B., Ortolani S., Momany Y., Hill V., Zoccali M., Renzini A., Minniti D., Pasquini L., Bica E., Rich R. M., VLT-UVES analysis of 5 giants in 47 Tucanae, A\&A, 2005, vol. 435, p. 657

Anderson J., Sarajedini A., Bedin L. R., King I. R., Piotto G., Reid I. N., Siegel M., Majewski S. R., Paust N. E. Q., Aparicio A., Milone A. P., Chaboyer B., Rosenberg A., The Acs Survey of Globular Clusters. V. Generating a Comprehensive Star Catalog for each Cluster, AJ, 2008, vol. 135, p. 2055

Armandroff T. E., The Properties of the Disk System of Globular Clusters, Astrophysical Journal, 1989, vol. 97 , p. 375

Asplund M., Grevesse N., Sauval A. J., Scott P., The Chemical Composition of the Sun, ARA\&A, 2009, vol. 47, p. 481

Barbuy B., Chiappini C., Cantelli E., Depagne E., Pignatari M., Hirschi R., Cescutti G., Ortolani S., Hill V., Zoccali M., Minniti D., Trevisan M., Bica E., Gómez A., Highresolution abundance analysis of red giants in the globular cluster NGC 6522, A\&A, 2014, vol. 570, p. A76

Barbuy B., Chiappini C., Gerhard O., Chemodynamical History of the Galactic Bulge, Annual Review of Astronomy and Astrophysics, 2018, vol. 56, p. 223

Barbuy B., Trevisan J., de Almeida A., Calculation of molecular line intensity in stellar atmospheres, PASA, 2018, vol. 35, p. 46 
Barmby P., Huchra J. P., M31 Globular Clusters in the Hubble Space Telescope Archive. I. Cluster Detection and Completeness, AJ, 2001, vol. 122, p. 2458

Bastian N., Lardo C., Multiple Stellar Populations in Globular Clusters, ARA\&A, 2018, vol. 56 , p. 83

Baumgardt H., Hilker M., A catalogue of masses, structural parameters, and velocity dispersion profiles of 112 Milky Way globular clusters, MNRAS, 2018, vol. 478, p. 1520

Bedin L. R., Piotto G., Anderson J., King I. R., Cassisi S., Momany Y., The double main sequence of Omega Centauri, Memorie della Societa Astronomica Italiana Supplementi, 2004, vol. 5, p. 105

Bica E., Alloin D., Schmitt H. R., Integrated spectral properties of star clusters in the near-ultraviolet., Astronomy \& Astrophysics, 1994, vol. 283, p. 805

Bica E., Bonatto C., Barbuy B., Ortolani S., Globular cluster system and Milky Way properties revisited, A\&A, 2006, vol. 450, p. 105

Bica E., Ortolani S., Barbuy B., Globular Clusters in the Galactic Bulge, Publications of the Astronomical Society of Australia, 2016, vol. 33

Bica E., Westera P., Kerber L. d. O., Dias B., Maia F., Santos João F. C. J., Barbuy B., Oliveira R. A. P., An Updated SMC and Magellanic Bridge Catalog of Star Clusters, Associations and Related Objects, arXiv e-prints, 2019, p. arXiv:1907.08642

Brogaard K., VandenBerg D. A., Bedin L. R., Milone A. P., Thygesen A., Grundahl F., The age of 47 Tuc from self-consistent isochrone fits to colour-magnitude diagrams and the eclipsing member V69, MNRAS, 2017, vol. 468, p. 645

Burstein D., Faber S., Gaskell C., Krumm N., Old stellar populations. I. A spectroscopic comparison of galactic globular clusters, M31 globular clusters, and elliptical galaxies., Astrophysical Journal, 1984, vol. 287, p. 586

Campbell S. W., D’Orazi V., Yong D., Constantino T. N., Lattanzio J. C., Stancliffe R. J., Angelou G. C., Wylie-de Boer E. C., Grundahl F., VizieR Online Data Catalog: Na abundances in NGC6752 (Campbell+, 2013), VizieR Online Data Catalog (other), 2013, vol. 0380, p. J/other/Nat/498 
Carretta E., Empirical estimates of the Na-O anti-correlation in 95 Galactic globular clusters, A\&A, 2019, vol. 624, p. A24

Carretta E., Bragaglia A., Gratton R., Lucatello S., Na-O anticorrelation and HB. VIII. Proton-capture elements and metallicities in 17 globular clusters from UVES spectra, A\&A, 2009, vol. 505, p. 139

Castelli F., Kurucz R. L., , 2004 New Grids of ATLAS9 Model Atmospheres

Chmielewski Y., The infrared triplet lines of ionized calcium as a diagnostic tool for F, G, K-type stellar atmospheres, A\&A, 2000, vol. 353, p. 666

Colaboração Gaia Gaia Data Release 2. Summary of the contents and survey properties, A\&A, 2018, vol. 616, p. A1

Colucci J. E., Bernstein R. A., McWilliam A., Globular Cluster Abundances from Highresolution, Integrated-light Spectroscopy. II. Expanding the Metallicity Range for Old Clusters and Updated Analysis Techniques, ApJ, 2017, vol. 834, p. 105

Conroy C., Villaume A., van Dokkum P. G., Lind K., Metal-rich, Metal-poor: Updated Stellar Population Models for Old Stellar Systems, ApJ, 2018, vol. 854, p. 139

Cordero M. J., Pilachowski C. A., Johnson C. I., McDonald I., Zijlstra A. A., Simmerer J., Detailed Abundances for a Large Sample of Giant Stars in the Globular Cluster 47 Tucanae (NGC 104), ApJ, 2014, vol. 780, p. 94

Denissenkov P. A., VandenBerg D. A., Kopacki G., Ferguson J. W., Constraints on the Distance Moduli, Helium, and Metal Abundances, and Ages of Globular Clusters from Their RR Lyrae and Non-variable Horizontal Branch Stars. II. Multiple Stellar Populations in 47 Tuc, M3, and M13, ApJ, 2017, vol. 849, p. 159

Dickens R. J., Bell R. A., Gustafsson B., Carbon and nitrogen abundances in red giant stars in the globular cluster 47 Tucanae., Astrophysical Journal, 1979, vol. 232, p. 428

Dotter A., Chaboyer B., Jevremović D., Kostov V., Baron E., Ferguson J. W., The Dartmouth Stellar Evolution Database, ApJS, 2008, vol. 178, p. 89 
Girardi L., Bressan A., Bertelli G., Chiosi C., VizieR Online Data Catalog: Low-mass stars evolutionary tracks \&amp; isochrones (Girardi+, 2000), VizieR Online Data Catalog, 2000, pp J/A+AS/141/371

Gratton R., Sneden C., Carretta E., Abundance Variations Within Globular Clusters, ARA\&A, 2004, vol. 42, p. 385

Gratton R. G., Bragaglia A., Carretta E., Clementini G., Desidera S., Grundahl F., Lucatello S., Distances and ages of NGC 6397, NGC 6752 and 47 Tuc, A\&A, 2003, vol. 408, p. 529

Gratton R. G., Carretta E., Bragaglia A., Multiple populations in globular clusters. Lessons learned from the Milky Way globular clusters, A\&A Rev., 2012, vol. 20, p. 50

Graves G. J., Schiavon R. P., Measuring Ages and Elemental Abundances from Unresolved Stellar Populations: Fe, Mg, C, N, and Ca, ApJS, 2008, vol. 177, p. 446

Gray D. F., The Observation and Analysis of Stellar Photospheres, 2005

Gustafsson B., Edvardsson B., Eriksson K., Jørgensen U. G., Nordlund Å., Plez B., A grid of MARCS model atmospheres for late-type stars. I. Methods and general properties, A\&A, 2008, vol. 486, p. 951

Harris W. E., Globular cluster systems in galaxies beyond the Local Group., Annual Review of Astronomy and Astrophysics, 1991, vol. 29, p. 543

Harris W. E., A Catalog of Parameters for Globular Clusters in the Milky Way., Astronomical Journal, 1996, vol. 112, p. 1487

Hinkle K., Wallace L., Valenti J., Harmer D., Visible and Near Infrared Atlas of the Arcturus Spectrum 3727-9300 A, 2000

Koch A., McWilliam A., A New Abundance Scale for the Globular Cluster 47 Tuc, AJ, 2008, vol. 135 , p. 1551

Kroupa P., On the variation of the initial mass function, MNRAS, 2001, vol. 322, p. 231

Kurucz R. L., New atlases for solar flux, irradiance, central intensity, and limb intensity, Memorie della Societa Astronomica Italiana Supplementi, 2005a, vol. 8, p. 189 
Kurucz R. L., New atlases for solar flux, irradiance, central intensity, and limb intensity, Memorie della Societa Astronomica Italiana Supplementi, 2005b, vol. 8, p. 189

La Barbera F., Ferreras I., Vazdekis A., de la Rosa I. G., de Carvalho R. R., Trevisan M., Falcón-Barroso J., Ricciardelli E., SPIDER VIII - constraints on the stellar initial mass function of early-type galaxies from a variety of spectral features, MNRAS, 2013, vol. 433 , p. 3017

Lagioia E., Milone A., Marino A., Cassisi S., Aparicio A., Piotto G., Anderson J., Barbuy B., Bedin L., Bellini A., et al., The Hubble Space Telescope UV Legacy Survey of Galactic Globular Clusters-XII. The RGB bumps of multiple stellar populations, Monthly Notices of the Royal Astronomical Society, 2018, vol. 475, p. 4088

Larsen S. S., Brodie J. P., Strader J., Detailed abundances from integrated-light spectroscopy: Milky Way globular clusters, A\&A, 2017, vol. 601, p. A96

Mann A. W., Feiden G. A., Gaidos E., Boyajian T., von Braun K., How to Constrain Your M Dwarf: Measuring Effective Temperature, Bolometric Luminosity, Mass, and Radius, ApJ, 2015, vol. 804, p. 64

Martins L. P., Lima-Dias C., Coelho P. R. T., Laganá T. F., Testing stellar population fitting ingredients with Globular Clusters I: Stellar libraries, MNRAS, 2019, vol. 484, p. 2388

McWilliam A., Bernstein R. A., Globular Cluster Abundances from High-Resolution Integrated-Light Spectra. I. 47 Tuc, ApJ, 2008, vol. 684, p. 326

McWilliam A., Preston G. W., Sneden C., Shectman S., A Spectroscopic Analysis of 33 of the Most Metal-Poor Stars.I., AJ, 1995, vol. 109, p. 2736

Meléndez J., Barbuy B., Bica E., Zoccali M., Ortolani S., Renzini A., Hill V., GeminiPhoenix infrared high-resolution abundance analysis of five giants in the bulge globular cluster NGC 6553, A\&A, 2003, vol. 411, p. 417

Milone A., Marino A. F., Bedin L. R., Anderson J., The HST large programme on Centauri - I. Multiple stellar populations at the bottom of the main sequence probed in NIROptical, Monthly Notices of the Royal Astronomical Society, 2017, vol. 469, p. 800 
Milone A. P., Marino A. F., Bedin L. R., Anderson J., Apai D., Bellini A., Dieball A., Salaris M., Libralato M., Nardiello D., Bergeron P., Burgasser A. J., Rees J. M., Rich R. M., Richer H. B., The HST Large Programme on NGC 6752 - II. Multiple populations at the bottom of the main sequence probed in NIR, MNRAS, 2019, vol. 484, p. 4046

Moura T. C., Trevisan M., Barbuy B., Rossi S., Integrated Spectra of Milky Way Globular Clusters, ApJ, 2019, vol. 885, p. 28

Norris J., Cottrell P. L., The nitrogen anomaly in 47 Tucanae., Astrophysical Journal, 1979, vol. 229, p. L69

Ortolani S., Renzini A., Gilmozzi R., Marconi G., Barbuy B., Bica E., Rich R. M., Nearcoeval formation of the Galactic bulge and halo inferred from globular cluster ages, Nature, 1995, vol. 377, p. 701

Pancino E., Bellazzini M., Giuffrida G., Marinoni S., Globular clusters with Gaia, MNRAS, 2017, vol. 467 , p. 412

Pehlivan Rhodin A., Hartman H., Nilsson H., Jönsson P., Experimental and theoretical oscillator strengths of Mg I for accurate abundance analysis, A\&A, 2017, vol. 598, p. A102

Pietrinferni A., Cassisi S., Salaris M., Castelli F., A Large Stellar Evolution Database for Population Synthesis Studies. I. Scaled Solar Models and Isochrones, ApJ, 2004, vol. 612, p. 168

Pietrinferni A., Cassisi S., Salaris M., Castelli F., A Large Stellar Evolution Database for Population Synthesis Studies. II. Stellar Models and Isochrones for an $\alpha$-enhanced Metal Distribution, ApJ, 2006, vol. 642, p. 797

Pilachowski C. A., Canterna R., Wallerstein G., The chemical compositions of stars in the globular cluster 47 Tucanae and the old disk cluster NGC 2420. , Astrophysical Journal, 1980, vol. 235, p. L21

Piotto G., Milone A., Bedin L., Anderson J., King I., Marino A., Nardiello D., Aparicio A., Barbuy B., Bellini A., et al., The Hubble Space Telescope UV Legacy Survey of 
Galactic Globular Clusters. I. Overview of the project and detection of multiple stellar populations, The Astronomical Journal, 2015, vol. 149, p. 91

Plez B., , 2012 Turbospectrum: Code for spectral synthesis

Prugniel P., Vauglin I., Koleva M., The atmospheric parameters and spectral interpolator for the MILES stars, A\&A, 2011, vol. 531, p. A165

Puzia T. H., Saglia R. P., Kissler-Patig M., Maraston C., Greggio L., Renzini A., Ortolani S., Integrated spectroscopy of bulge globular clusters and fields. I. The data base and comparison of individual Lick indices in clusters and bulge, A\&A, 2002, vol. 395, p. 45

Pérez-Villegas A., Barbuy B., Kerber L., Ortolani S., Souza S. O., Bica E., Globular clusters in the inner Galaxy classified from dynamical orbital criteria, Monthly Notices of the Royal Astronomical Society, 2019

Roediger J. C., Courteau S., Graves G., Schiavon R. P., Constraining Stellar Population Models. I. Age, Metallicity and Abundance Pattern Compilation for Galactic Globular Clusters, ApJS, 2014, vol. 210, p. 10

Ryabchikova T., Piskunov N., Kurucz R. L., Stempels H. C., Heiter U., Pakhomov Y., Barklem P. S., A major upgrade of the VALD database, Phys. Scr, 2015, vol. 90, p. 054005

Salasnich B., Girardi L., Weiss A., Chiosi C., VizieR Online Data Catalog: Evolution models for $\alpha$-enhanced stars (Salasnich+ 2000), VizieR Online Data Catalog, 2000, pp $\mathrm{J} / \mathrm{A}+\mathrm{A} / 361 / 1023$

Salpeter E. E., The Luminosity Function and Stellar Evolution., ApJ, 1955, vol. 121, p. 161

Sánchez-Blázquez P., Peletier R. F., Jiménez-Vicente J., Cardiel N., Cenarro A. J., FalcónBarroso J., Gorgas J., Selam S., Vazdekis A., Medium-resolution Isaac Newton Telescope library of empirical spectra, MNRAS, 2006, vol. 371, p. 703

Sarajedini A., Bedin L. R., Chaboyer B., Dotter A., Siegel M., Anderson J., Aparicio A., King I., Majewski S., Marín-Franch A., Piotto G., Reid I. N., Rosenberg A., The ACS 
Survey of Galactic Globular Clusters. I. Overview and Clusters without Previous Hubble Space Telescope Photometry, AJ, 2007, vol. 133, p. 1658

Schiavon R. P., Faber S. M., Rose J. A., Castilho B. V., Population Synthesis in the Blue. II. The Spectroscopic Age of 47 Tucanae, ApJ, 2002, vol. 580, p. 873

Schiavon R. P., Rose J. A., Courteau S., MacArthur L. A., A Library of Integrated Spectra of Galactic Globular Clusters, ApJS, 2005, vol. 160, p. 163

Shapley H., Studies based on the colors and magnitudes in stellar clusters. VII. The distances, distribution in space, and dimensions of 69 globular clusters., Astrophysical Journal, 1918, vol. 48, p. 154

Sharma K., Prugniel P., Singh H. P., New atmospheric parameters and spectral interpolator for the MILES cool stars, A\&A, 2016, vol. 585, p. A64

Smith G., Drake J. J., Collisional broadening of the calcium infrared triplet lines by atomic hydrogen, MNRAS, 1988, vol. 231, p. 115

Sneden C., The nitrogen abundance of the very metal-poor star HD 122563., ApJ, 1973, vol. 184, p. 839

Souza S. O., Kerber L. O., Barbuy B., Pérez-Villegas A., Oliveira R. A. P., Nardiello D., Self-consistent analysis of stellar clusters: An application to HST data of the halo globular cluster NGC 6752, arXiv e-prints, 2020, p. arXiv:2001.02697

Thompson I. B., Kaluzny J., Rucinski S. M., Krzeminski W., Pych W., Dotter A., Burley G. S., The Cluster AgeS Experiment (CASE). IV. Analysis of the Eclipsing Binary V69 in the Globular Cluster 47 Tuc, AJ, 2010, vol. 139, p. 329

Thygesen A. O., Sbordone L., Andrievsky S., Korotin S., Yong D., Zaggia S., Ludwig H. G., Collet R., Asplund M., Ventura P., D'Antona F., Meléndez J., D'Ercole A., The chemical composition of red giants in 47 Tucanae. I. Fundamental parameters and chemical abundance patterns, A\&A, 2014, vol. 572, p. A108

Usher C., Beckwith T., Bellstedt S., Alabi A., Chevalier L., Pastorello N., Cerulo P., Dalgleish H. S., Fraser-McKelvie A., Kamann S., Penny S., Foster C., McDermid R., Schiavon R. P., Villaume A., The WAGGS project - II. The reliability of the calcium 
triplet as a metallicity indicator in integrated stellar light, MNRAS, 2019, vol. 482, p. 1275

Usher C., Pastorello N., Bellstedt S., Alabi A., Cerulo P., Chevalier L., Fraser-McKelvie A., Penny S., Foster C., McDermid R. M., Schiavon R. P., Villaume A., The WAGGS project - I. The WiFeS Atlas of Galactic Globular cluster Spectra, MNRAS, 2017, vol. 468, p. 3828

VandenBerg D. A., Bergbusch P. A., Ferguson J. W., Edvardsson B., Isochrones for Old (\&gt;5 Gyr) Stars and Stellar Populations. I. Models for -2.4 \&lt; $=[\mathrm{Fe} / \mathrm{H}] \& \mathrm{lt} ;=+0.6$, $0.25 \& \mathrm{lt} ;=\mathrm{Y} \& \mathrm{lt} ;=0.33$, and $-0.4 \& \mathrm{lt} ;=[\alpha / \mathrm{Fe}] \& \mathrm{lt} ;=+0.4, \mathrm{ApJ}, 2014$, vol. 794 , p. 72

VandenBerg D. A., Brogaard K., Leaman R., Casagrand e L., The Ages of 55 Globular Clusters as Determined Using an Improved VĤB_TO Method along with ColorMagnitude Diagram Constraints, and Their Implications for Broader Issues, ApJ, 2013, vol. 775 , p. 134

Wylie E. C., Cottrell P. L., Sneden C. A., Lattanzio J. C., Heavy-Element Abundances in Giant Stars in 47 Tucanae, ApJ, 2006, vol. 649, p. 248 
Apêndice 



\section{Apêndice A}

\section{Comparação entre espectros do SynSSP e espectro observado em Usher et al. (2017)}

As Figuras a seguir apresentam uma comparação, no intervalo 4500 - $9000 \AA$, do espectro construído pelo SynSSP e do espectro apresentado em Usher et al. (2017) para o 47 Tuc. A linha vermelha representa o espectro sintético e os pontos pretos representam o espectro empírico. 

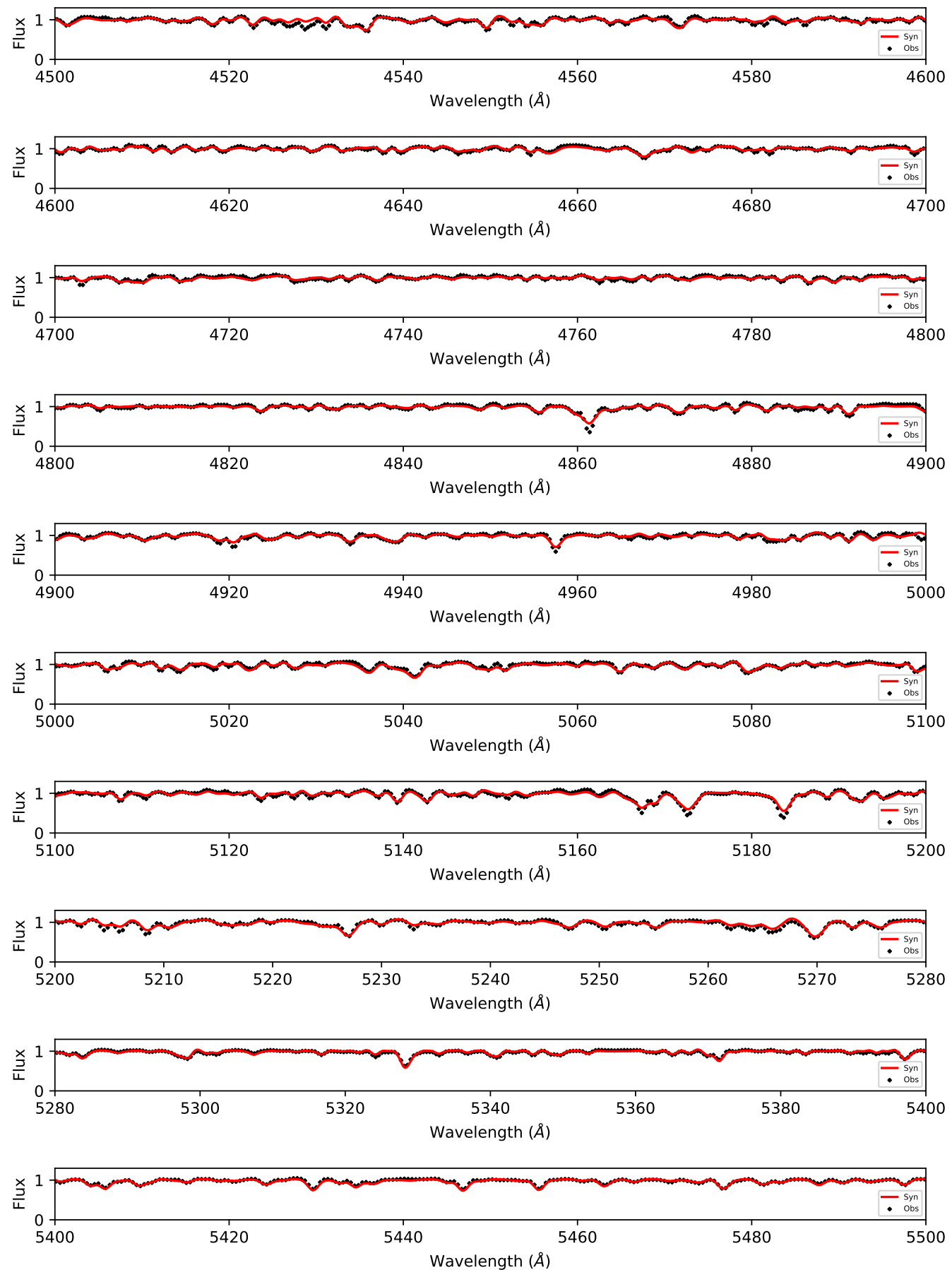

Figura A.1: Comparação entre entre os espectros sintético e observado, no intervalo 4500 - $5500 \AA$. 

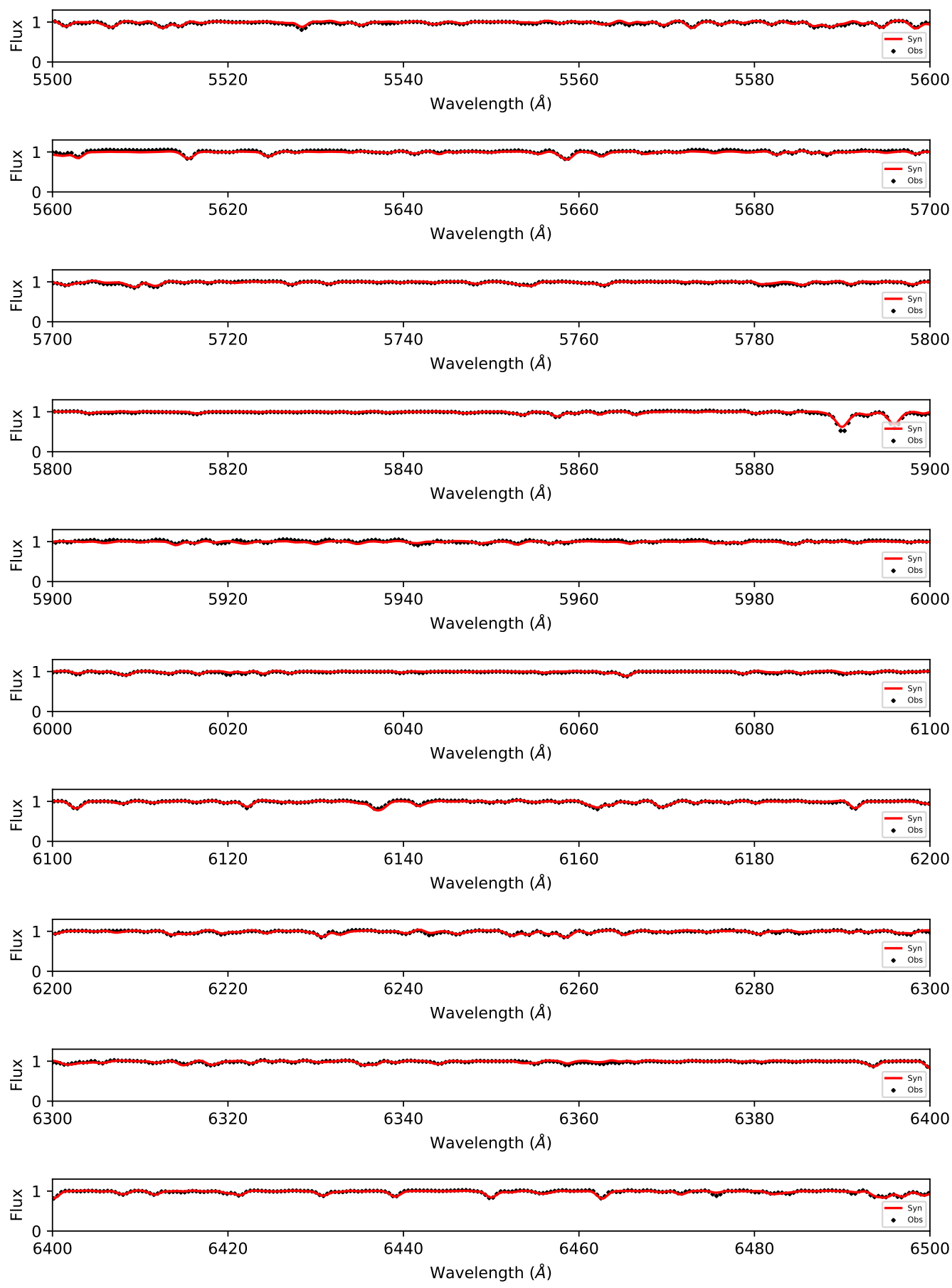

Figura A.2: Continuação da figura A.1, comparação entre entre os espectros sintético e observado, no intervalo $5500-6500 \AA$. 

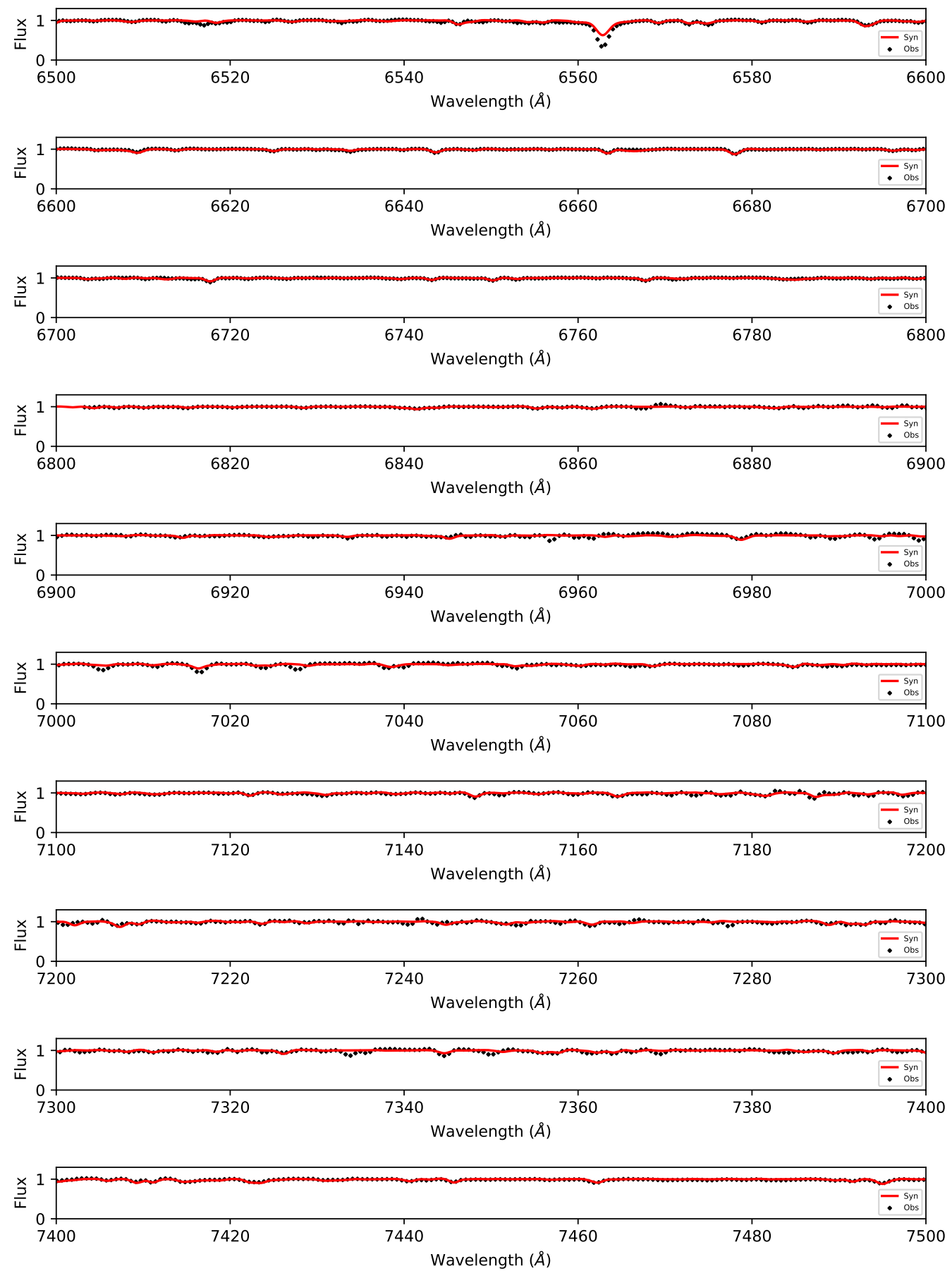

Figura A.3: Continuação da figura A.2, comparação entre entre os espectros sintético e observado, no intervalo $6500-7500 \AA$. 

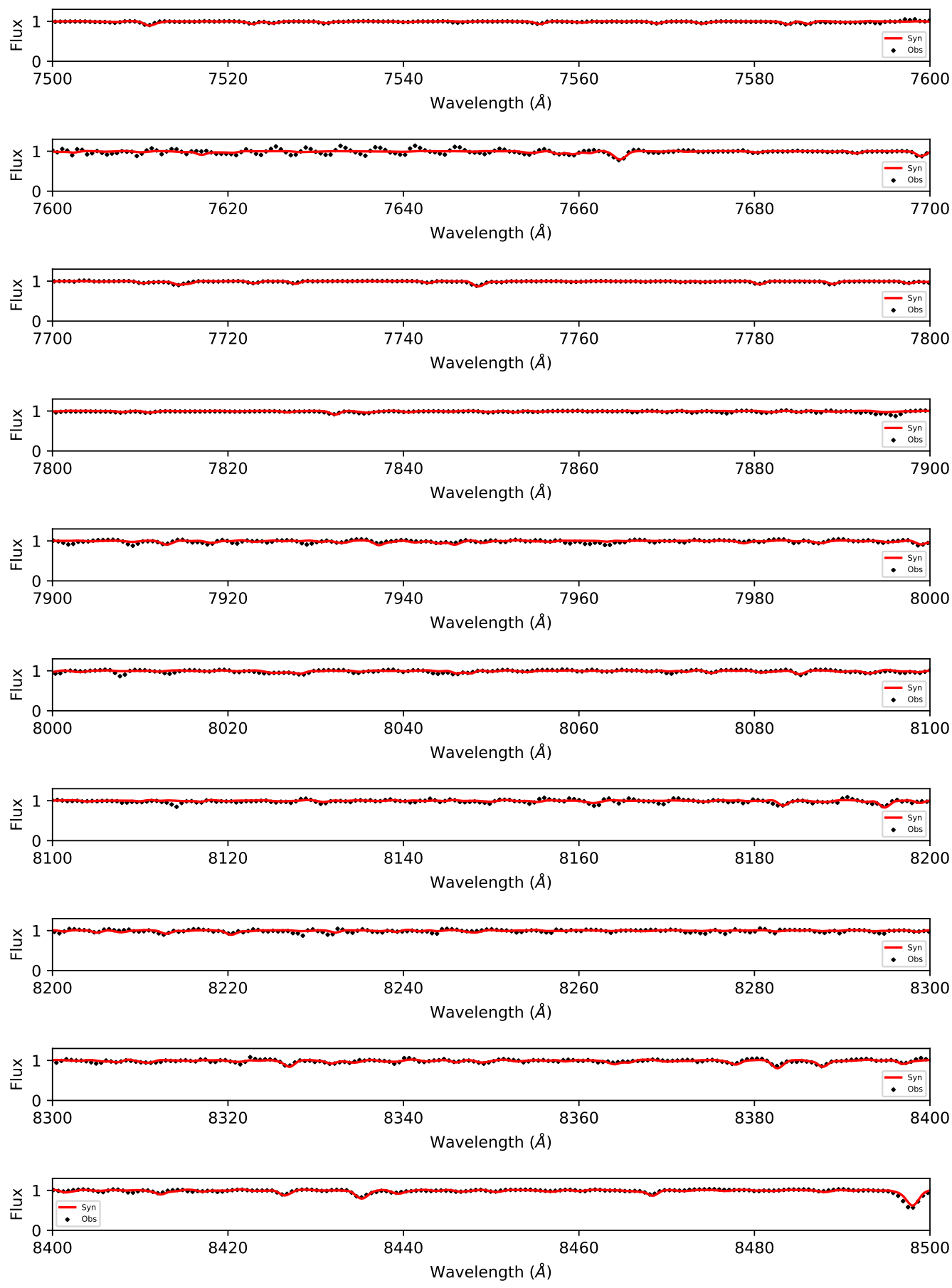

Figura A.4: Continuação da figura A.3, comparação entre entre os espectros sintético e observado, no intervalo $7500-8500 \AA$. 

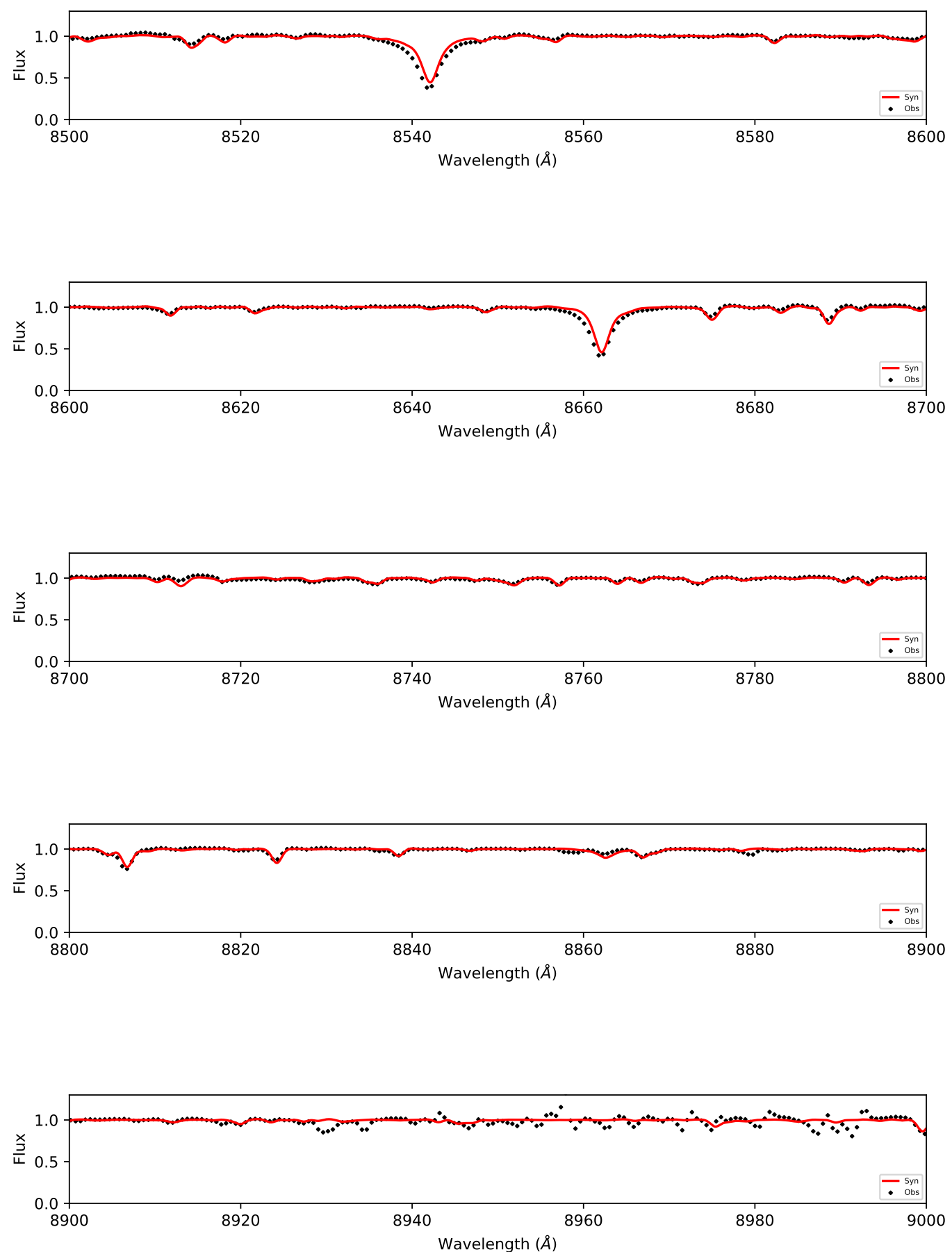

Figura A.5: Continuação da figura A.4, comparação entre entre os espectros sintético e observado, no intervalo $8500-9000 \AA$. 


\section{Apêndice B}

\section{Comparação entre espectros sintéticos com idades de 11 e 13 Gyr, e espectro empírico}

As Figuras a seguir apresentam uma comparação, no intervalo 4500 - $9000 \AA$, entre os espectros construídos pelo SynSSP, para idades de 11 e 13 Gyrs. A linha vermelha representa o espectro sintético para a idade de 13 Gyrs, os pontos pretos representam o espectro sintético para a idade de 11 Gyrs, e os pontos em azul representam o espectro empírico apresentado em Usher et al. (2017). Abaixo de cada quadro de 100 Å, está uma comparação entre os espectros sintéticos, que foi calculada por meio das diferenças de fluxos entre eles. Note que esse valor nunca ultrapassa o valor de 0.05, ou seja, a diferença entre eles é sempre igual ou inferior a $5 \%$. 

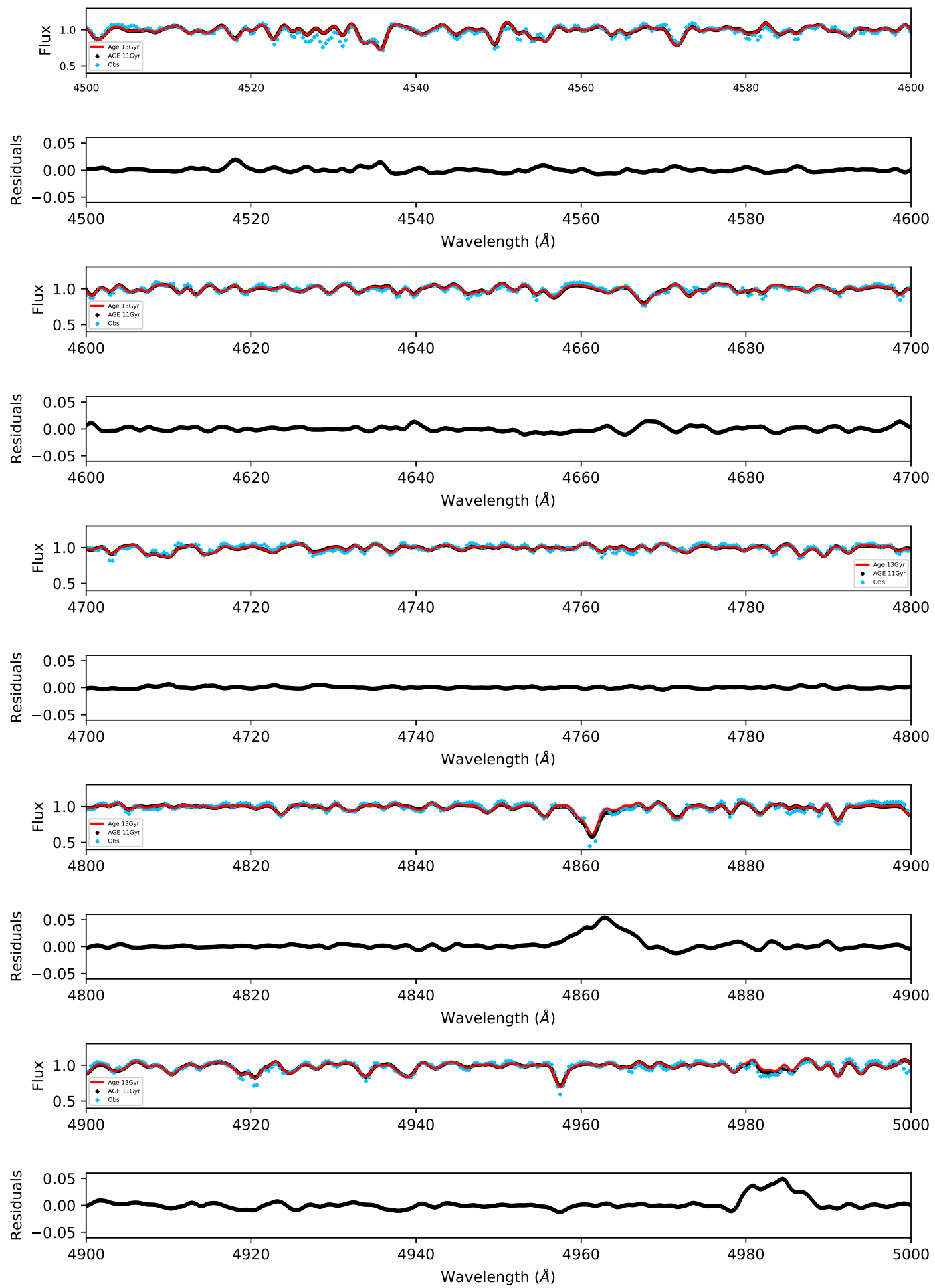

Figura B.1: Espectros sintéticos com idades de 11 e 13 Gyr e espectro observado sobrepostos, no intervalo $4500-5000 \AA$. 

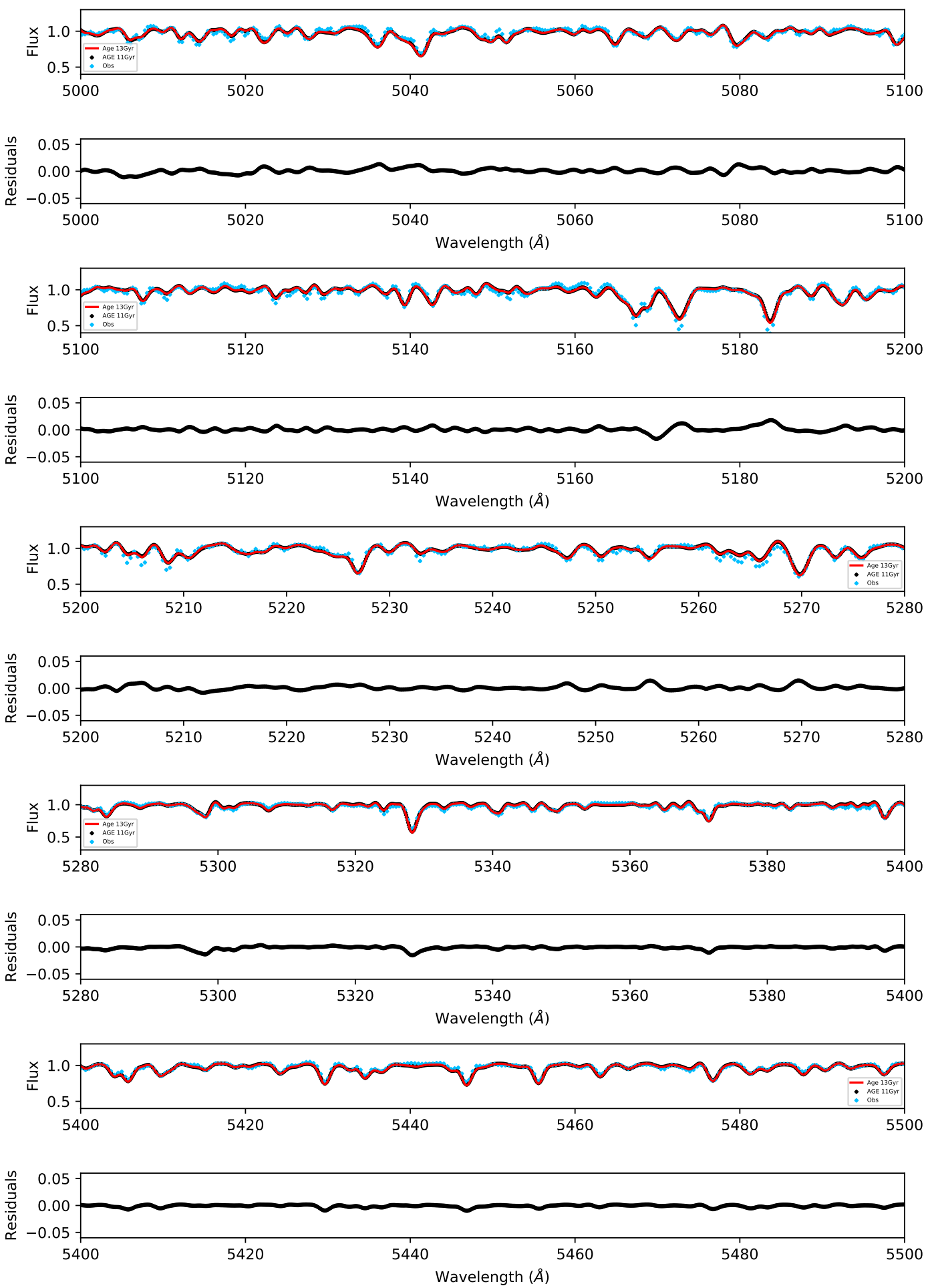

Figura B.2: Espectros sintéticos com idades de 11 e 13 Gyr e espectro observado sobrepostos, no intervalo $5000-5500 \AA$. 

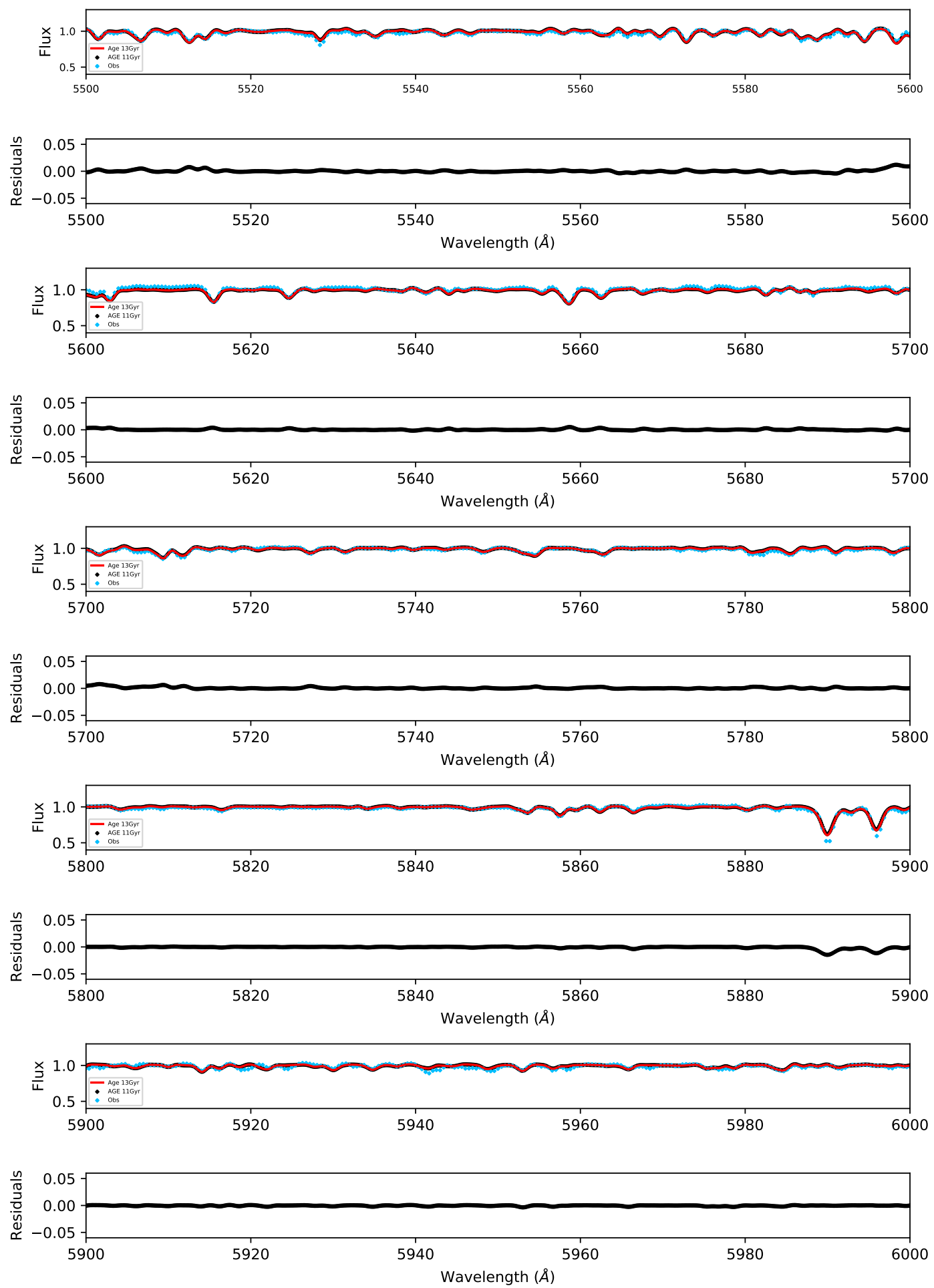

Figura B.3: Espectros sintéticos com idades de 11 e 13 Gyr e espectro observado sobrepostos, no intervalo $5500-6000 \AA$. 

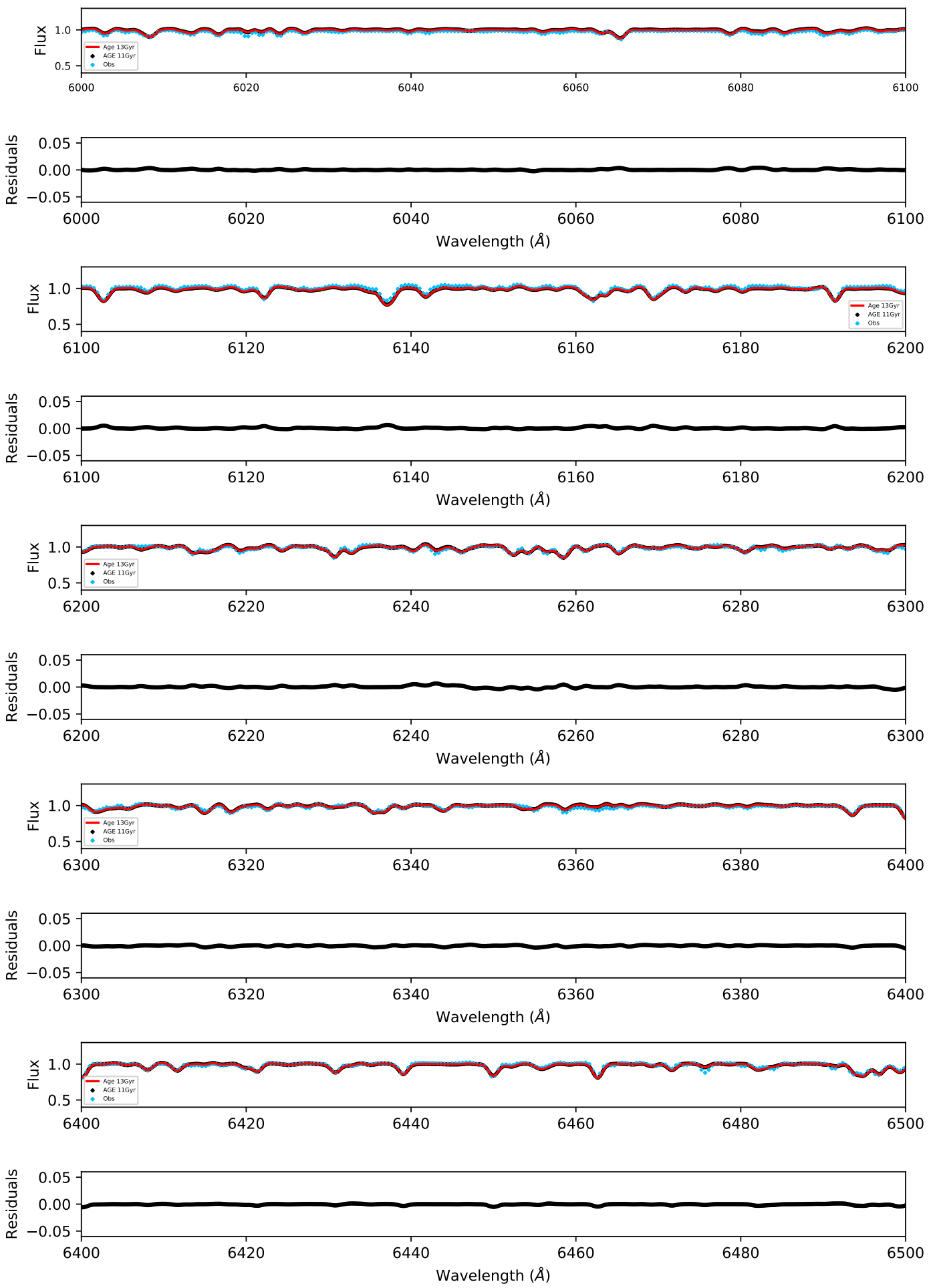

Figura B.4: Espectros sintéticos com idades de 11 e 13 Gyr e espectro observado sobrepostos, no intervalo $6000-6500 \AA$. 

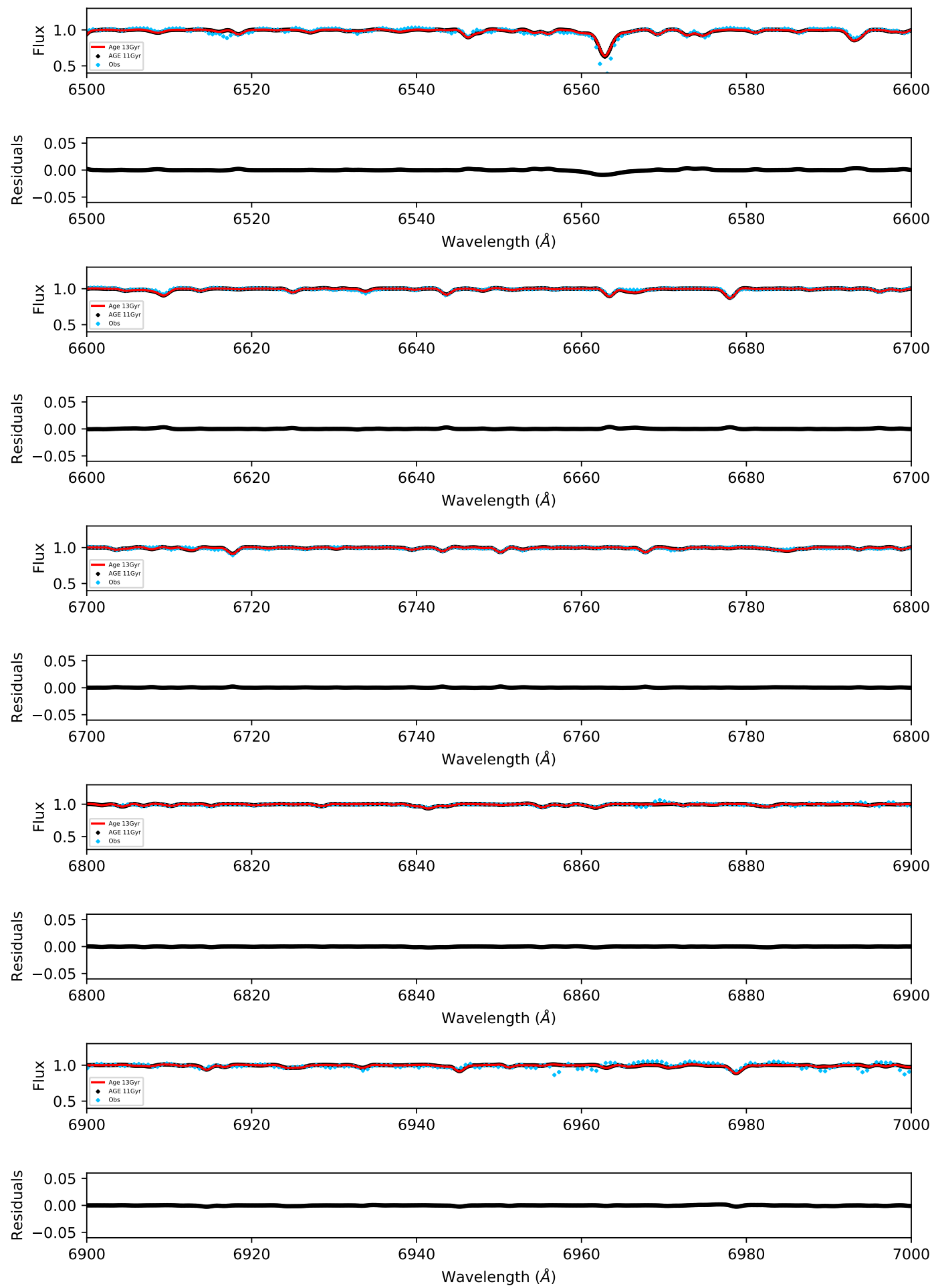

Figura B.5: Espectros sintéticos com idades de 11 e 13 Gyr e espectro observado sobrepostos, no intervalo $6500-7000 \AA$. 

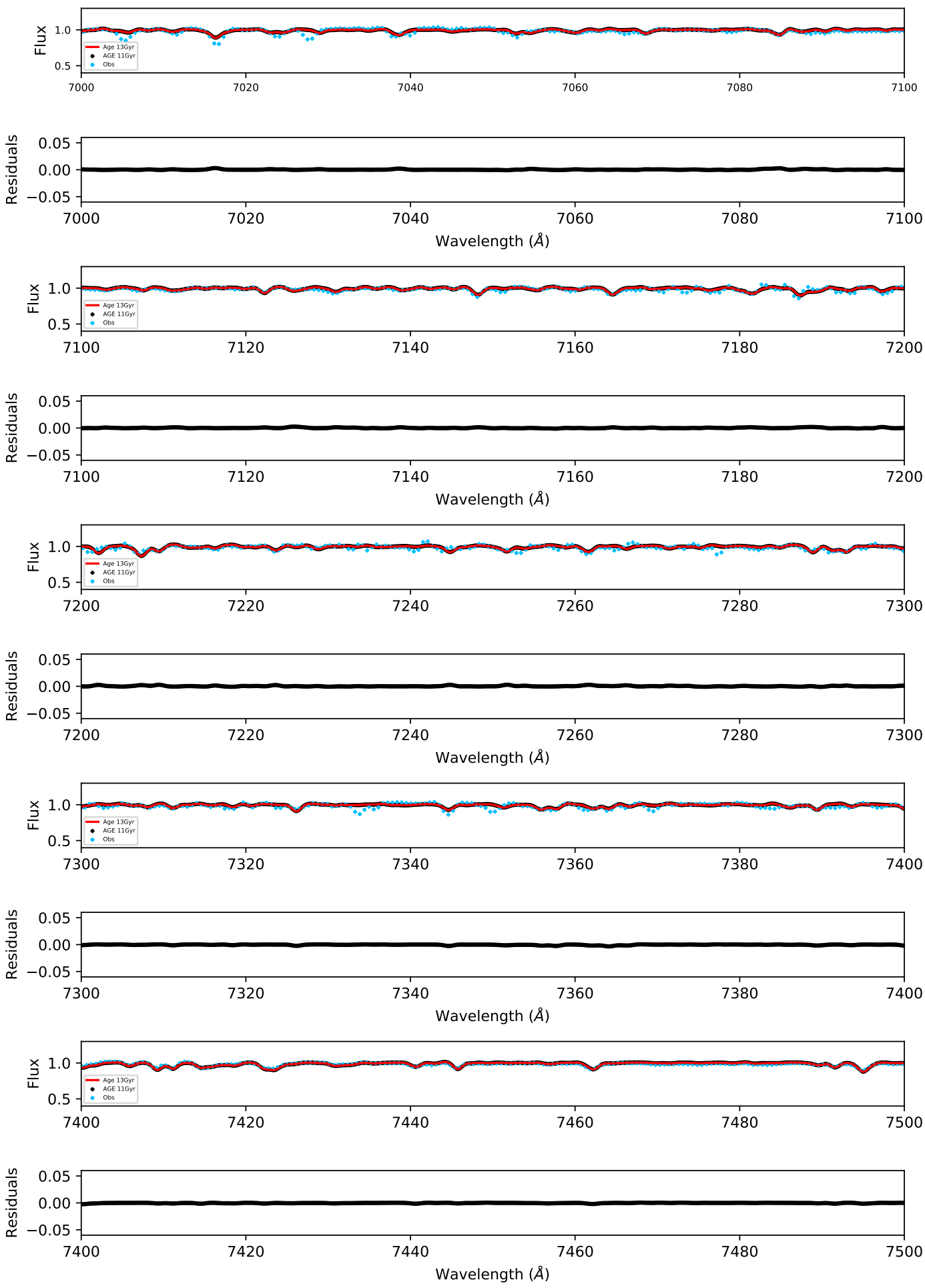

Figura B.6: Espectros sintéticos com idades de 11 e 13 Gyr e espectro observado sobrepostos, no intervalo $7000-7500 \AA$. 

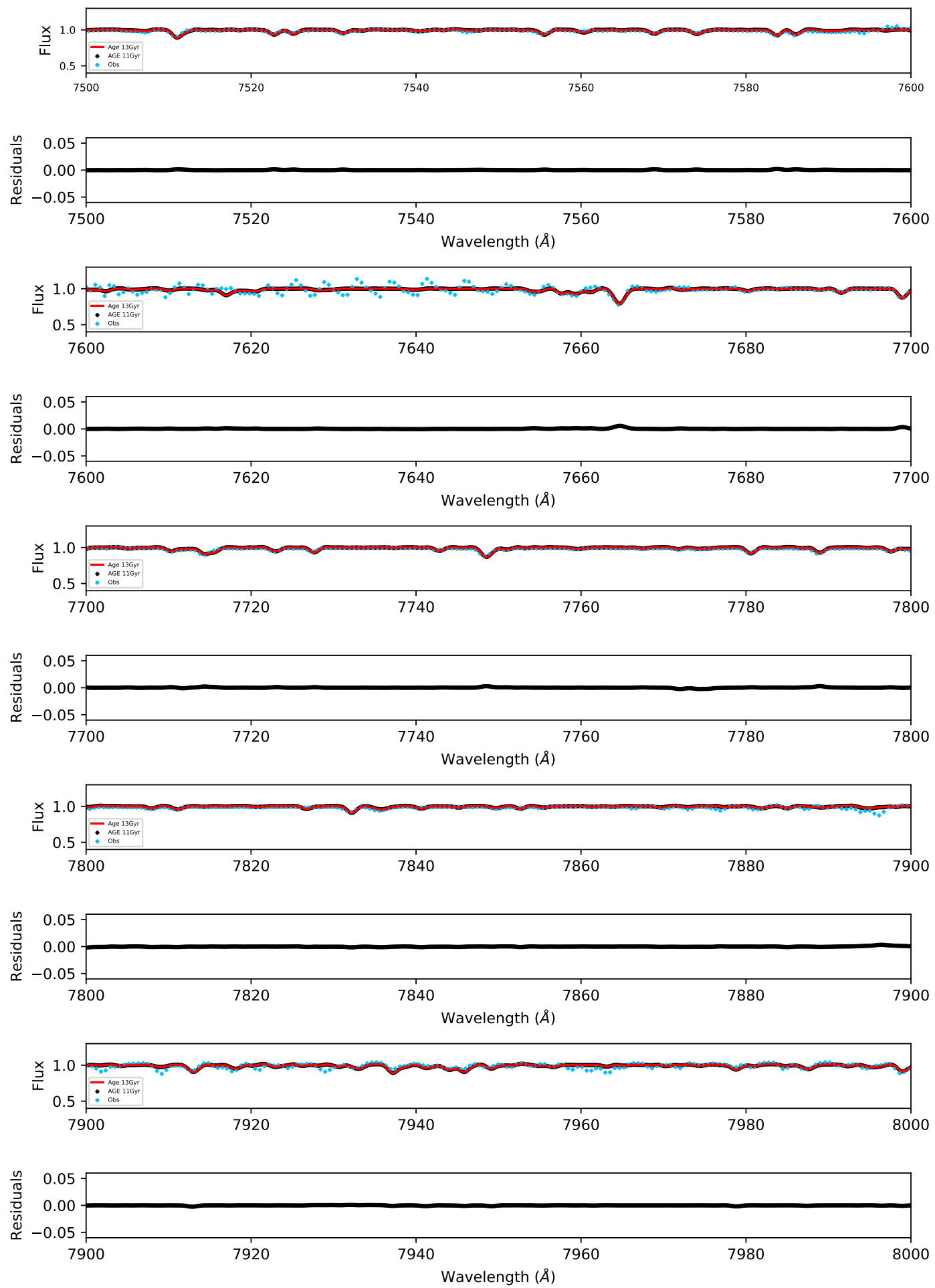

Figura B.7: Espectros sintéticos com idades de 11 e 13 Gyr e espectro observado sobrepostos, no intervalo $7500-8000 \AA$. 

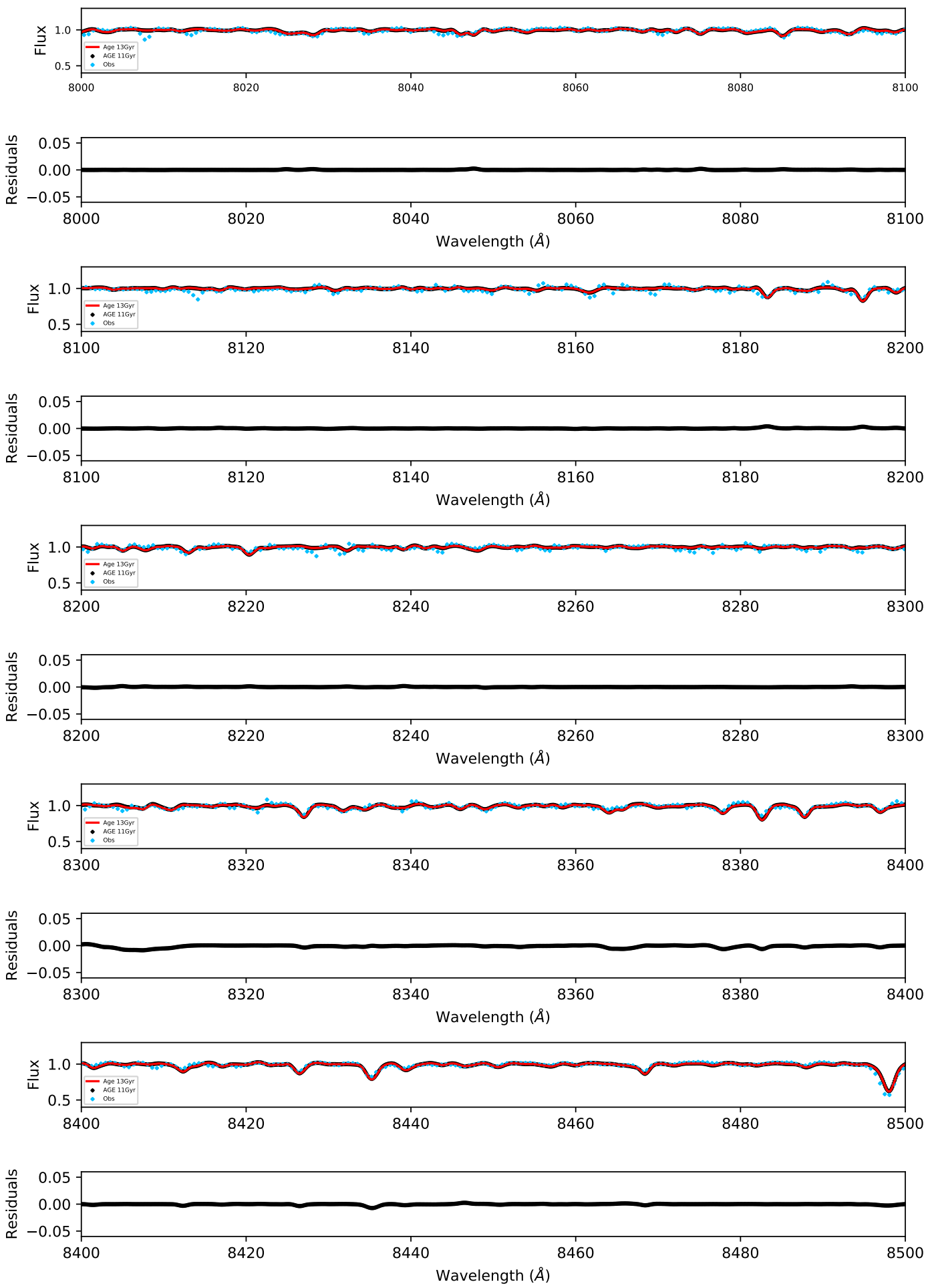

Figura B.8: Espectros sintéticos com idades de 11 e 13 Gyr e espectro observado sobrepostos, no intervalo $8000-8500 \AA$. 

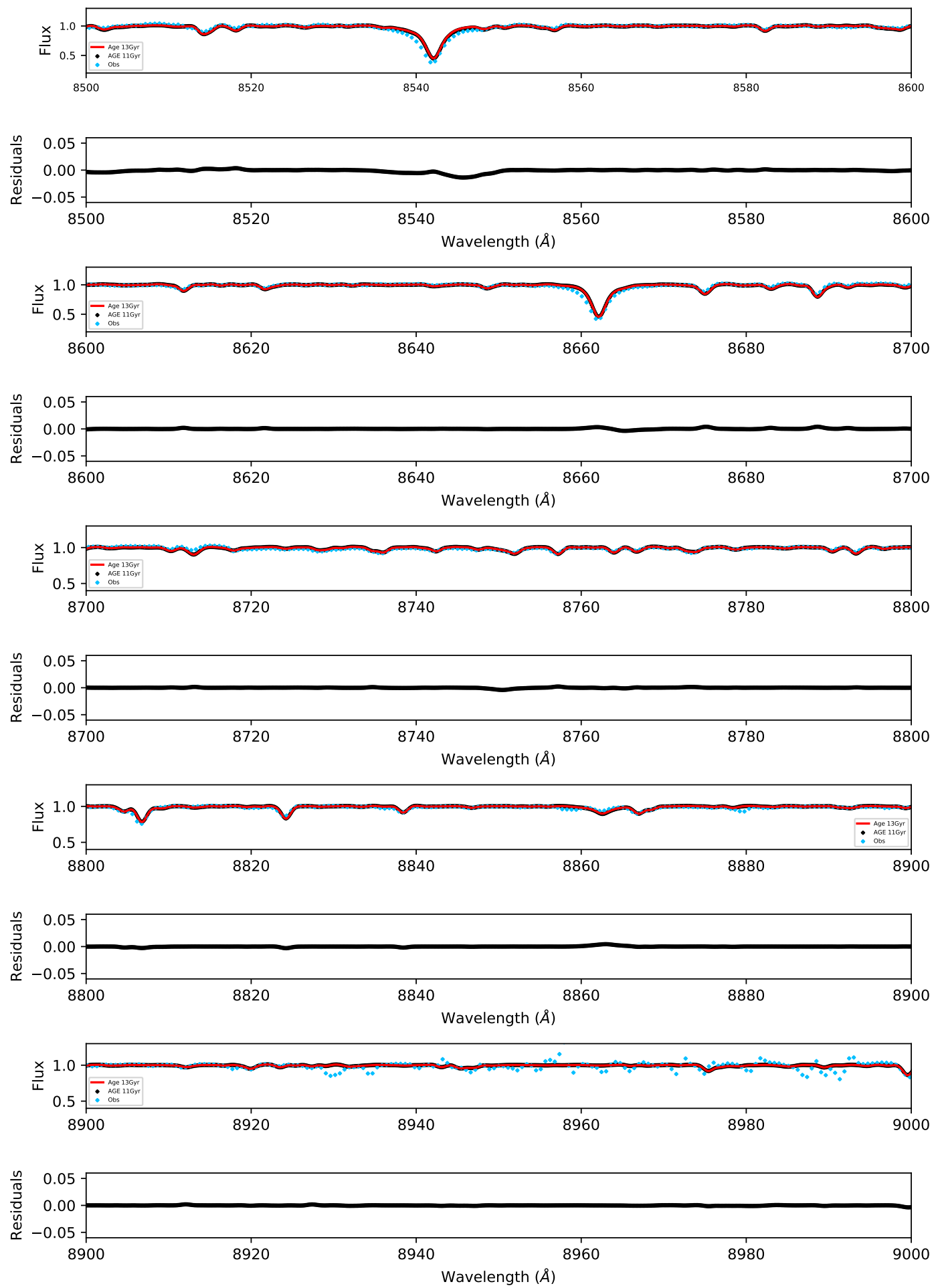

Figura B.9: Espectros sintéticos com idades de 11 e 13 Gyr e espectro observado sobrepostos, no intervalo $8500-9000 \AA$. 